\title{
SUB-2 NM PLATINUM NANOPARTICLES GROWTH STUDY AND DEVICE APPLICATIONS
}

A Dissertation presented to the Faculty of the Graduate School at the University of Missouri-Columbia

In Partial Fulfillment

of the Requirements for the Degree

Doctor of Philosophy

by

BALAVINAYAGAM RAMALINGAM

Dr. Shubhra Gangopadhyay, Thesis Supervisor

May 2014 
The undersigned, appointed by the dean of the Graduate School, have examined the dissertation entitled

\section{SUB-2 NM PLATUNUM NANOPARTICLES GROWTH STUDY AND DEVICE APPLICATIONS}

Presented by Balavinayagam Ramalingam, A candidate for the degree of Doctor of Philosophy in Electrical and Computer Engineering

And hereby certify that, in their opinion, it is worthy of acceptance.

Professor Shubhra Gangopadhyay (Chairperson of the Committee)

Professor Scott Kovaleski

Professor Sathish Nair

Professor Mark Lee 


\section{ACKNOWLEDGEMENTS}

I wish to offer my sincere gratitude to my advisor, Dr. Shubhra Gangopadhyay, for her wonderful guidance and support throughout my graduate career. I appreciate her impeccable knowledge in the subject matter and her attitude for encouraging students to think independently over the research problem and build up skills to work as a team. Her thoughtful advice in my professional and personal life has shaped me as a good researcher and a better person. I would also like to thank my committee members, Dr. Scott Kovaleski, Dr. Sathish Nair and Dr. Mark Lee for their sincere advice on my research work at every step. Next, I would like to extend my gratitude to University of Missouri- Research Reactor and Dr. John D. Brockmann for helping me out during the device and exposure studies. I would also like to thank Dr. Keshab Gangopadhyay for having very useful discussions and helping me in every aspect during the course of my research; and Dr. Joseph C Mathai for training and mentoring in device fabrication and troubleshooting problems encountered in any steps.

I would like to acknowledge my current and past group members including Somik Mukherjee, Haisheng Zheng, Yun Minseong, Chase Darr, Steven C Hamm, Sagnik Basuray, Venu Korampally, Yang Zhou, Clay Staley and Rajagopalan Thiruvengadam who made my research work very enjoyable and my stay a memorable experience. I would also like to thank Janice Rudeen, Shirley Holdmeir, Audrey Clark and office member of the College of Engineering Dean's office for helping with the administrative work and support. Finally, I would like to thank Dr. Kai Song from Missouri University of Science and Technology (Rolla, MO) for his expert advice in nanoparticle HRTEM imaging. 


\section{TABLE OF CONTENTS}

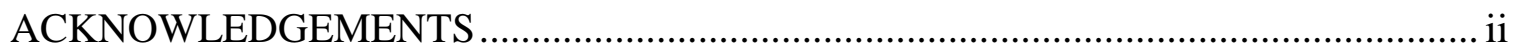

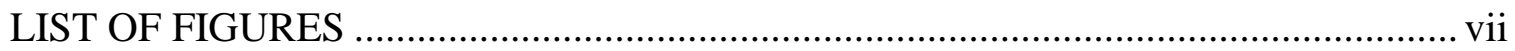

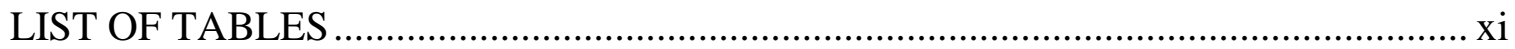

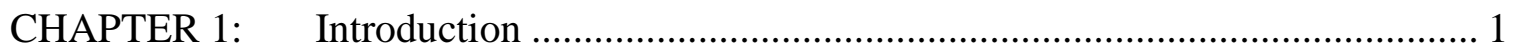

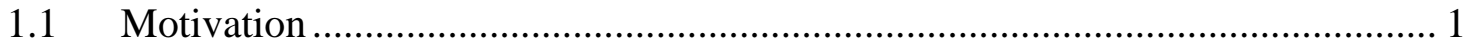

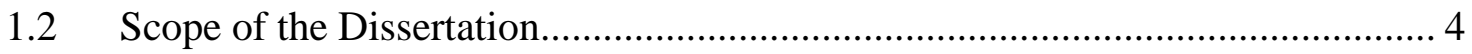

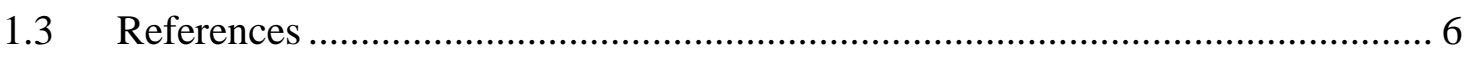

CHAPTER 2: $\quad$ Sub-2 nm Platinum Nanoparticle Growth Study ............................... 15

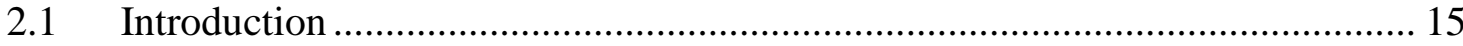

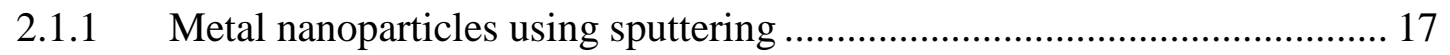

2.2 Substrate Surface Energy and Thin Film Growth Modes ............................... 18

2.2.1 Tilted Target Deposition ................................................................. 21

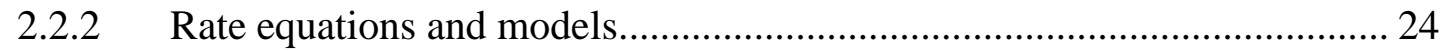

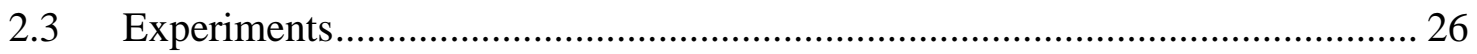

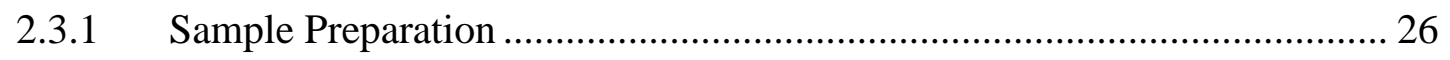

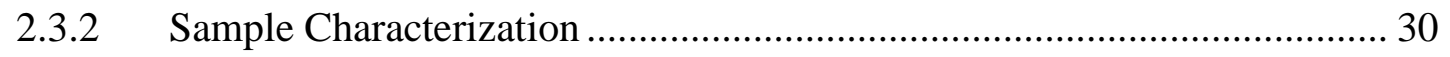

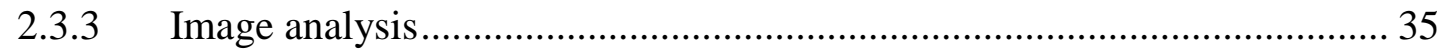

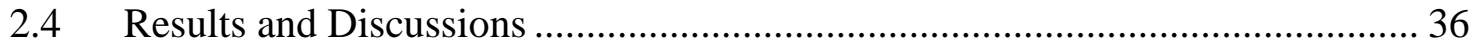




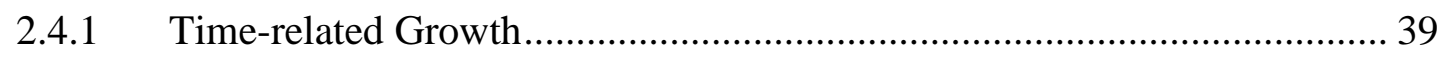

2.4.2 Homogenous size distribution of sputtered nanoparticles ....................... 43

2.4.3 Target angle dependent nanoparticle growth ......................................... 46

2.4.4 Time dependent growth at different target angles ................................ 48

2.4.5 Substrate location variation in deposition plume ................................... 57

2.4.6 Sputtering power dependent growth ............................................... 59

2.4.7 Development of size dependent nanoparticle crystallinity ....................... 65

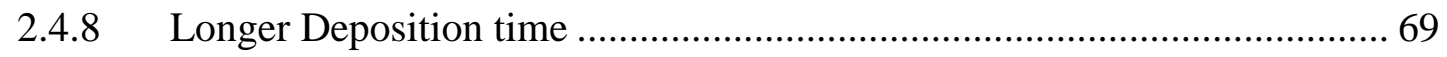

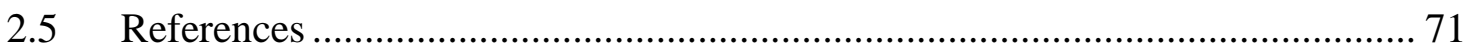

CHAPTER 3: $\quad$ Layer By Layer Charging In Multilevel Pt Nanoparticle Embedded Non

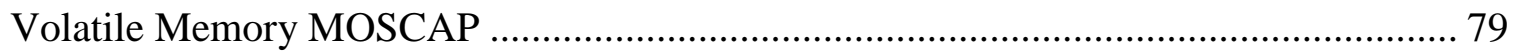

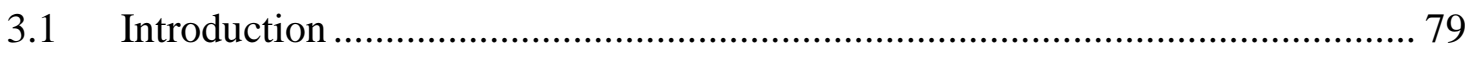

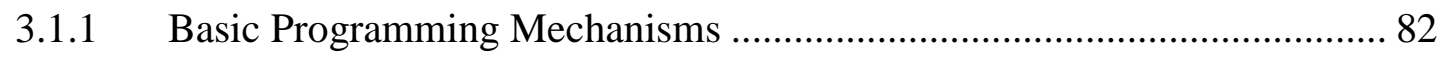

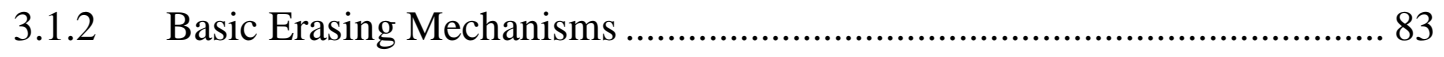

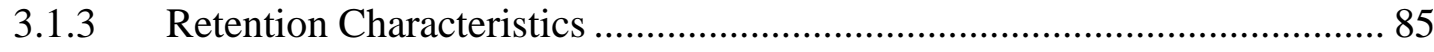

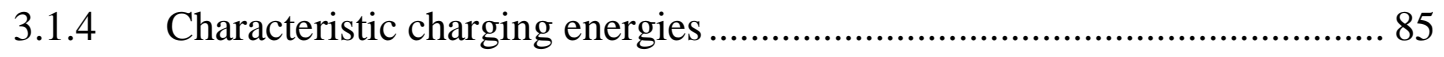

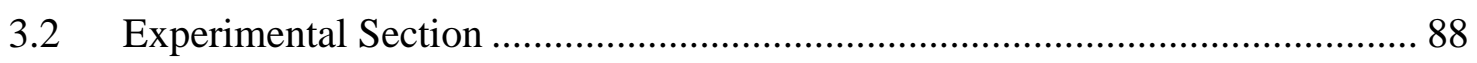

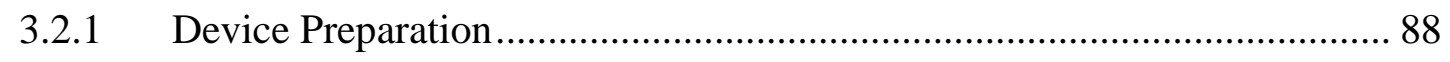

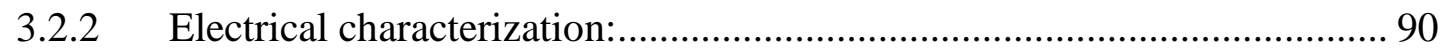

3.2.3 Particle deposition and TEM characterization: .................................... 90 
3.3.1 Pt Nanoparticle Configuration . 98

3.3.2 Demonstrating Controlled Nanoparticle Layer Charging through Capacitance-Voltage Measurement 100

3.3.3 Effect of thinner tunneling layer and larger diameter first layer PtNP ..... 105

3.3.4 Effect of varying the second layer Pt NP density ............................... 108

3.3.5 Effect of a thicker separation layer ................................................ 111

3.3.6 Avoiding lateral tunneling and Coulomb staircase .............................. 113

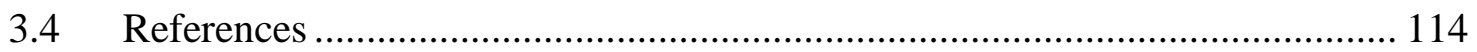

CHAPTER 4: $\quad$ Neutron Detectors with integrated Pt NP and ${ }^{10} \mathrm{~B}$.......................... 120

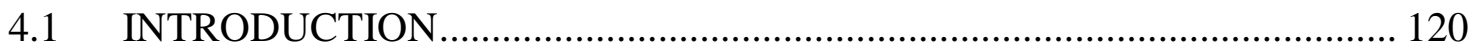

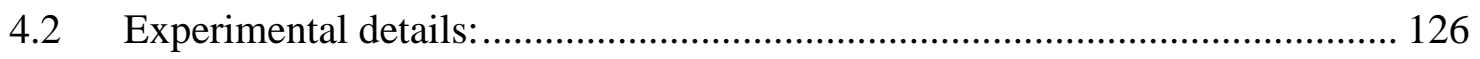

4.2.1 Device Fabrication ...................................................................... 126

4.2.2 Neutron Exposure .................................................................. 131

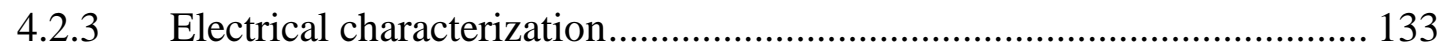

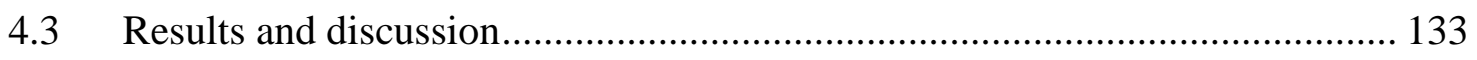

4.3.1 Neutron exposure on Samples with PS/DB capping layer and no embedded Pt NP: 133

4.3.2 Samples with PS/DB capping layer and embedded Single layer Pt NP: .. 141 
4.3.3 Neutron exposure on Samples with PS/DB capping layer and embedded dual layer Pt NP:

4.3.4 Charge Calculations and Detector efficiency: ......................................... 152

4.3.5 Ionization damage and displacement effects .......................................... 156

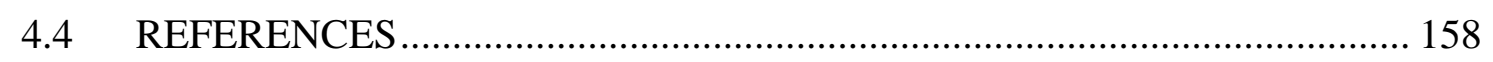

CHAPTER 5: Conclusions and Future Work …………………………………...... 162

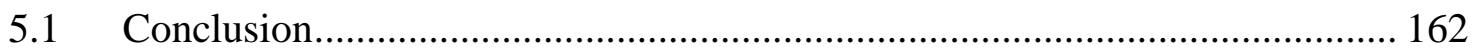

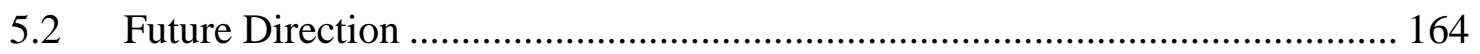

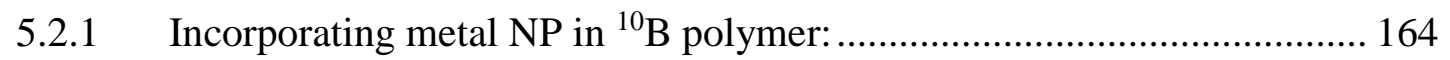

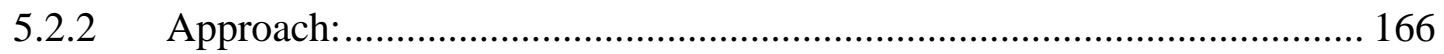

5.2.3 Silicon on Insulator Devices and sputtered InGaZnO .............................. 167

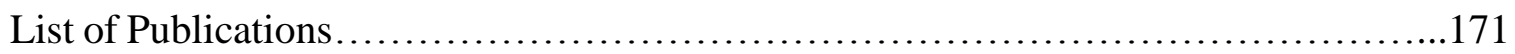

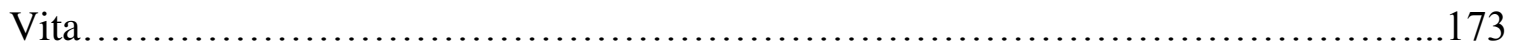




\section{LIST OF FIGURES}

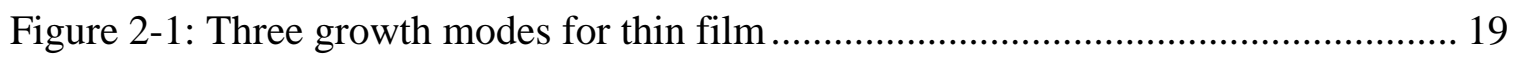

Figure 2-2: Representation of deposition plume for tilted target deposition .................... 23

Figure 2-3: Particle growth phenomena demonstrating nucleation, growth, coalescence and

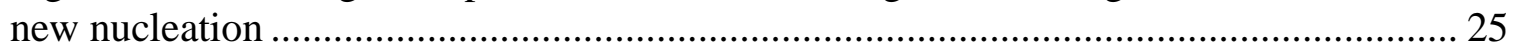

Figure 2-4: AJA Sputtering tool used for nanoparticle fabrication ................................... 26

Figure 2-5: (a) Sputter chamber set-up with tilt-target configuration representing an interpretation of different spatial parameters in the sputtering chamber, which are involved in the nanoparticle growth process. The remaining images (b-d) show atom flux with tilttarget configuration at (b) $16.2^{\circ}$ (c) $23.8^{\circ}$ and (d) $38.8^{\circ}$. 29

Figure 2-6: 3-D profile of $\mathrm{Al}_{2} \mathrm{O}_{3}$ surface roughness and inset table surface showing roughness characteristics measured using AFM. 31

Figure 2-7: (a) HRTEM of Pt nanoparticles on $\mathrm{Al}_{2} \mathrm{O}_{3}$ with less than $5 \mathrm{~s}$ beam exposure and (b) with greater than $10 \mathrm{~s}$ beam exposure. (The white circles highlight the presence of extremely small Pt nanoparticles). 34

Figure 2-8: Schematic showing the different growth stages (a) Nucleation, (b) Coalescence and (c) Agglomeration. 37

Figure 2-9: HRTEM images of Pt nanoparticles deposited at $23.8^{\circ}$ for (a) $5 \mathrm{~s}$, (b) $10 \mathrm{~s}$, (c) $20 \mathrm{~s}$, (d) $30 \mathrm{~s}$, (e) $45 \mathrm{~s}$ and (f) $55 \mathrm{~s}$. Plot of (g) mean diameter vs. deposition time and (h) mean density vs. deposition time. Linear-fit modeling (rate equation) of time-related growth mode (i). 42

Figure 2-10: Particle size distribution for $30 \mathrm{~W}$ deposition power (target angle=23.80) and for $5 \mathrm{~s}, 10 \mathrm{~s}, 15 \mathrm{~s}, 20 \mathrm{~s}, 25 \mathrm{~s}, 30 \mathrm{~s}, 35 \mathrm{~s}, 40 \mathrm{~s}, 45 \mathrm{~s}, 50 \mathrm{~s}$ and $55 \mathrm{~s}$. 45

Figure 2-11: Plot showing (a) mean diameter and (b) density with different target angles 47

Figure 2-12: (a) mean diameter plot (TA=16.2 deg); (b) density (TA=16.2 deg); (c) 2 min at TA=16.2 deg; (d) $2 \mathrm{~min}$ at TA=23.8 deg; (e) $2 \mathrm{~min}$ at TA=38.8deg; (f) Mean Diameter for TA=38.8 deg and (g) Density for TA=38.8 deg.

Figure 2-13:TEM images for Pt Nanoparticles deposited using 16.2 target angle and varying deposition time for a deposition power of $30 \mathrm{~W}$ and (a) $10 \mathrm{~s}$, (b) 20s, (c) $30 \mathrm{~s}$, (d) $45 \mathrm{~s}$ and (e) $120 \mathrm{~s}$ 
Figure 2-14: Size distribution for Pt Nanoparticles deposited using 16.2 target angle and varying deposition time for a deposition power of 30W and (a) 10s, (b) 20s, (c) 30s, (d) $45 \mathrm{~s}$ and (e) $120 \mathrm{~s}$.

Figure 2-15: TEM images for Pt Nanoparticles deposited using 38.8 target angle and varying deposition time for a deposition power of 30W and (a) 10s, (b) 20s, (c) 30s, (d) $45 \mathrm{~s}$ and (e) $120 \mathrm{~s}$. 55

Figure 2-16:Size distribution for Pt Nanoparticles deposited using 38.8 target angle and varying deposition time for a deposition power of 30W and (a) 10s, (b) 20s, (c) 30s and (d) $45 \mathrm{~s}$. 56

Figure 2-17: Plot for samples at different substrate location: (a) mean diameter plot and (b) Density plot. 58

Figure 2-18: (a) Mean Diameter and (b) density plot vs. Deposition Power (at Deposition time $=10 \mathrm{~s}$ and $\mathrm{TA}=23.8 \mathrm{deg}$ ) 60

Figure 2-19:TEM images for Pt Nanoparticles deposited using varying power at deposition time of $10 \mathrm{~s}$ and target angle of $23.8^{\circ}$ for (a) $20 \mathrm{~W}$, (b) $24 \mathrm{~W}$, (c) $27 \mathrm{~W}$, (d) $30 \mathrm{~W}$, (e) $40 \mathrm{~W}$, (f) $50 \mathrm{~W}$ and $(\mathrm{g}) 60 \mathrm{~W}$

Figure 2-20: Particle size distribution for $20 \mathrm{~W}, 24 \mathrm{~W}, 27 \mathrm{~W}, 30 \mathrm{~W}, 40 \mathrm{~W}, 50 \mathrm{~W}$ and $60 \mathrm{~W}$ with a deposition time of $10 \mathrm{~s}(\mathrm{TA}=23.8)$. 64

Figure 2-21:HRTEM images of Pt nanoparticles sputtered for various durations: (a) $10 \mathrm{~s}$, (b) $20 \mathrm{~s}$, (c) $30 \mathrm{~s}$, (d) $45 \mathrm{~s}$, (e) $60 \mathrm{~s}$, and (f) $120 \mathrm{~s}$ with a deposition power of $30 \mathrm{~W}$ and TA $=23.8$.

Figure 2-22: HRTEM images of Pt nanoparticles prepared by sputtering onto $\mathrm{Al}_{2} \mathrm{O}_{3}$ alongside their corresponding FFT patterns (inset) for (a, b) $30 \mathrm{~s}$ and (c, d) $45 \mathrm{~s} \mathrm{Pt}$ sputtering. The corresponding FFT patterns represent an FCC crystal along the $\langle 110\rangle$ zone axis 68

Figure 2-23:HRTEM images for (a)2 min, (b)5 min, (c)10 min and (d)20 min deposition using 30W deposition power and 23.8 degree target angle 70

Figure 3-1: Energy band diagram FN tunneling for (a) Programming and (b) Erasing ... 84

Figure 3-2: (a) Plane view TEM image of a single-layer of uniformly distributed $1.08 \mathrm{~nm}$ Pt nanoparticles. The inset of (a) shows particle size distribution over 5 images after statistical post-image analysis (b) Plane view TEM for double layer Pt nanoparticles (c) Plane view TEM for triple layer Pt nanoparticles. (d) Plane view TEM for quadruple layer Pt nanoparticles 92

Figure 3-3 Plots from image analysis of multiple layers showing (a) Mean Diameter vs. Number of Layers and (b) Particle Density vs. Number of Layers. 93 
Figure 3-4: EDS Spectrum of (a) single-, (b) double-, (c) triple-, and (d) quad-layer Pt nanoparticle samples. (e) Integrated intensity of Pt M peak (f) Integrated intensity of Pt L peak

Figure 3-5: Cross-sectional TEM of quad layer sample. Inset shows the particle size distribution after statistical post-image analysis 97

Figure 3-6:(a) Schematic of double-layer NVM MOSCAP device designed for controlled charging. Plane view HRTEM for (b) $0.52 \pm 0.12 \mathrm{~nm}$ PtNP $\left(23.8^{\circ} / 5 \mathrm{~s}\right)$, (c) $1.11 \pm 0.28 \mathrm{~nm}$ $\operatorname{PtNP}\left(23.8^{\circ} / 20 \mathrm{~s}\right)$, and (d) $0.7 \pm 0.19 \mathrm{~nm} \operatorname{PtNP}\left(16.2^{\circ} / 20 \mathrm{~s}\right)$ 99

Figure 3-7: (a-d) CV characteristics for NVM MOSCAP with (a) no PtNP layer (control), (b) a single $0.52 \pm 0.12 \mathrm{~nm}$ PtNP layer over a $3 \mathrm{~nm}$ tunneling oxide layer, (c) a single 1.11 $\pm 0.28 \mathrm{~nm}$ PtNP layer over a $6 \mathrm{~nm}$ tunneling oxide layer, (d) the final double-layer device (3 nm tunneling layer | $0.52 \pm 0.12 \mathrm{~nm}$ PtNP layer $\mid 3 \mathrm{~nm}$ separation oxide | $1.11 \pm 0.28$ nm PtNP layer); (e) Flat band voltage as a function of gate voltage during program and erase conditions; and (f) Plot illustrating the step effect in the memory window as a function of gate voltage. 103

Figure 3-8 Retention characteristics for first layer charging and second layer charging with $7 \mathrm{~V}$ and $15 \mathrm{~V}$ respectively. The left axis denotes change in Flat Band and the right axis denotes the total charge loss in percentage. 104

Figure 3-9: MOSCAP NVM with (a) 2nm tunneling, $0.52 \pm 0.12 \mathrm{~nm}$ PtNP first layer, 3nm interparticle layer thickness, $1.11 \pm 0.28 \mathrm{~nm}$ Second layer PtNP and $15 \mathrm{~nm}$ capping oxide (inset: extracted Flat band voltage vs. gate voltage for erase and programming mode); (b) $3 \mathrm{~nm}$ tunneling, $0.7 \pm 0.196 \mathrm{~nm}$ PtNP second layer, 3nm interparticle layer thickness, 1.11 $\pm 0.28 \mathrm{~nm}$ second layer PtNP and $15 \mathrm{~nm}$ capping oxide. (inset: extracted Flat band voltage vs. gate voltage for erase and programming mode) 107

Figure 3-10: CV characteristics of MOSCAP NVM (a) dual layer Pt NP with 3nm tunneling $\mathrm{Al}_{2} \mathrm{O}_{3}$, first $\mathrm{NP}$ layer of $5 \mathrm{sec} \mathrm{Pt} \mathrm{NP}(\mathrm{TA}=23.8)$, 3nm separation $\mathrm{Al}_{2} \mathrm{O}_{3}$ layer and a low density Pt NP layer using 20s(TA=16.2 deg) and 15nm Capping oxide; (b) Second layer Pt NP (20s/TA=16.2) only with $7 \mathrm{~nm}$ tunneling and $15 \mathrm{~nm}$ capping oxide; (c) Change in $\mathrm{V}_{\mathrm{FB}}$ for program and erase modes and; (d) Extracted memory window ..... 110

Figure 3-11: Shows the CV characteristics and erase programming efficiency for a NVM MOSCAP with (a-b)3nm Tunneling, $5 \mathrm{sec} \mathrm{Pt} \mathrm{NP}(\mathrm{TA}=23.8)$, 4nm Separation $\mathrm{Al}_{2} \mathrm{O}_{3}$ layer, $20 \mathrm{sec}$ Pt NP(TA=23.8) and 15nm capping (c-d) 3nm Tunneling, $5 \mathrm{sec}$ Pt NP(TA=23.8), $4 \mathrm{~nm}$ Separation $\mathrm{Al}_{2} \mathrm{O}_{3}$ layer, $20 \mathrm{sec} \mathrm{Pt} \mathrm{NP}(\mathrm{TA}=16.2)$ and $15 \mathrm{~nm}$ capping .... 112

Figure 4-1 (a) Interpretation of neutron interaction in a Boron enriched converter layer (b) reaction products in 180 degree phase. 123

Figure 4-2: Device Schematic for Si based Pt NP embedded MOSCAP device (a)Dual layer Pt NP with ${ }^{10} \mathrm{~B}$ enriched Decaborane/Polystyrene capping layer (b) Dual Layer Pt NP embedded MOSCAP without Polystyrene/Decaborane layer, and (c)Single Layer Pt NP embedded MOSCAP with Polystyrene/Decaborane capping layer. 128 
Figure 4-3: Unshielded neutron source distribution plot

Figure 4-4: Different types of oxide charges

Figure 4-5: Frequency Dispersion C-V plot for (a) $20 \mathrm{~nm}$ Al2O3 Control sample without PS/DB capping and (b) $6 \mathrm{~nm}$ Al2O3 with 122nm PSDB capping oxide MOSCAP 138

Figure 4-6:C-V plots for Control sample with no embedded Pt NP with stack configurations of $\mathrm{Si} / 9 \mathrm{~nm} \mathrm{Al}_{2} \mathrm{O}_{3} / 122 \mathrm{~nm}$ PS/DB layer; exposed to neutron dose of (a) $0 \mathrm{n} / \mathrm{cm}^{2}(\mathrm{~b}$ ) $1.19 \mathrm{E} 10 \mathrm{n} / \mathrm{cm}^{2}$, (c) $1.615 \mathrm{E} 10 \mathrm{n} / \mathrm{cm}^{2}$, (d) $3.0175 \mathrm{E} 10 \mathrm{n} / \mathrm{cm}^{2}$ and (e) $5.1 \mathrm{E} 10 \mathrm{n} / \mathrm{cm}^{2}$.(f) shows the combination of all plots for comparison. 140

Figure 4-7: C-V plots for neutron exposure results for single layer Pt NP embedded MOSCAP with PS/DB layer embedded with (a)1.11 nm Pt NP and (b) $1.45 \mathrm{~nm}$ Pt ..... 148

Figure 4-8: C-V plots for Dual layer Pt NP sample exposed to neutron dose of (a) 0 $\mathrm{n} / \mathrm{cm}^{2}$ (b) $1.19 \mathrm{E} 10 \mathrm{n} / \mathrm{cm}^{2}$, (c) $1.615 \mathrm{E} 10 \mathrm{n} / \mathrm{cm}^{2}$, (d) $3.0175 \mathrm{E} 10 \mathrm{n} / \mathrm{cm}^{2}$ and (e) $5.1 \mathrm{E} 10 \mathrm{n} / \mathrm{cm}^{2}$.(f) shows the combination of all plots for comparison. 151

Figure 4-9: $V_{F B}$ shift comparison for dual layer vs Control sample for different neutron exposure.

Figure 4-10 (a) Representation of energetic particle interaction with NP embedded device stack; (b)Charge plot vs Neutron dose for experimental (blue, green) vs MCNPX estimations 155

Figure 5-1: SOI- based Boron and NP enriched MOSFET 167 


\section{LIST OF TABLES}

Table 2-1: Different sputter parameters used for Pt nanoparticle growth ....................... 28

Table 2-2: $\mathrm{Al}_{2} \mathrm{O}_{3}$ roughness parameter from AFM images ........................................ 31

Table 2-3: Pt Nanoparticle mean-diameter and its standard deviation with time variation. 44

Table 2-4: Pt Nanoparticle mean-diameter and its standard deviation with power variation 62

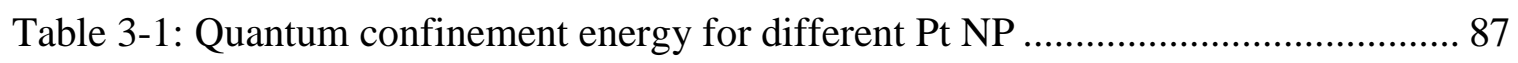

Table 3-2: Thickness and ALD process parameters for each $\mathrm{Al}_{2} \mathrm{O}_{3}$ layer...................... 89

Table 3-3: Pt Nanoparticle Size and Density Configuration at Different Layers ............. 90

Table 4-1: Different Layers with thickness and method of deposition......................... 129

Table 4-2 Pt NP size and its associated Coulomb charging energy ............................ 141

Table 4-3 Capacitance terms associated to the $0.5 \mathrm{~nm}$ Pt NP................................... 143

Table 4-4: Quantum confinement energy for different Pt NP ................................. 144

Table 4-5: Required Gate voltage to exhibit programming in devices with 122nm PS/DB

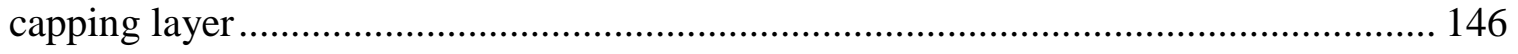

Table 4-6: Number of e-h pair estimation from MCNPX simulations for different neutron flux. 154 


\section{CHAPTER 1: Introduction}

\subsection{Motivation}

Nanoparticles and their potential applications are among the most pertinent subjects in the recent scientific community's research era. Some of the more promising nanoparticles are metal nanoparticles, offering a high degree of versatility in tuning specific physical and chemical properties for future uses in applications from chemical/biological sensors to use in applications involving energy storage and generation. Metal nanoparticles present the possibility of a wide range of uses because of their larger density of states at the Fermi energy, ability to tune the work function and the choice of metals specific for applications. They have attracted strong interest in applications over various field of study. Some of these applications include optical applications due to quantum size effects and enhancement of non-linear optical susceptibility [1-6], electrochemical applications for higher surface area-to-volume ratios $[\underline{7}, \underline{8}]$ and as floating gate memory devices for their charge storage capabilities [1-7].

Some of the most researched applications for metal nanoparticles is in the field of catalysis and non-volatile memory application. For catalysis applications, because of their increased surface/volume ratio compared to traditional thin films, metal nanoparticles results in more reaction sites for a given volume. Especially, Pt nanoparticles have been studied for their size dependent uses in oxidation of hydrocarbons, carbon di-oxide reduction and water hydrolysis. Thus, controlling the nanoparticle size is necessary to 
enable large surface areas and to produce an optimal size for catalysis [8]. Such properties for the Pt nanoparticles and their applications studied here are reported in [8-11].

This work particularly focuses on the growth of Pt nanoparticle and its other application as metal nanoparticle based Non Volatile Flash memory. Since the International Technology Roadmap for Semiconductors (ITRS) roadmap forecasts charge trapping as the solution to continue NAND flash scaling, it has become essential to identify advanced materials capable of enhancing retention properties, ideally at the nanoscale [12, 13]. Research has shown that discrete metal nanoparticles (MNPs) offer improved charge retention characteristics with respect to continuous charge trap layers due to the coulomb blockade effect and their ability to electrically isolate the nodes from their neighbors [1319]. Various kinds of memory devices have been produced using embedded MNPs to improve device characteristics, especially multiple floating gates for enhanced charge storage $[3,17,19,20]$. For these applications, both NP parameters - the size and the interparticle distance plays a key role in the charge storage and retention properties $[1,3$, 17, 21-24]. A good control of these properties can exhibit enhanced Coulomb blockade effects essential for charge storage. Thus again, controlling the size and the interparticle distance becomes an essential requirement for phenomenal improvements in the field of floating gate based non-volatile memories [1-3, 5, 6, 25, 26].

Several chemical routes have been investigated to obtain metal NPs including supercritical fluid deposition[27], chemical reduction[28-32], electrochemical deposition[33, 34], sol-gel processes[35, 36], and electroless plating[37, 38]. The major drawbacks of these techniques is that incorporating these nanoparticles in systems requiring subsequent top-down processing steps which are not straightforward and 
chemical synthesis often lead to undesirable side products. [39] To address these issues, small nanoparticles have been synthesized using top-down techniques such as physical vapour deposition (PVD) and chemical vapour deposition (CVD). However, the large variation in the nanoparticle size and number density as observed in these studies is not desirable for applications focusing on size dependent behaviour [7, 17, 40-46] .

Top-down approaches like de-wetting $[6,17,47]$ and direct deposition [40, 42, 48, 49] have also been used to create nanoparticles but has often limited control over its size, distribution and number density. Thus, in order to further reduce metal/Pt NP sizes, research into new techniques is needed and is currently an active area in nanotechnology research. Moreover, such research can open up numerous possibilities in the field of NVM flash memory devices and catalysis for making substantial improvement in their respective efficiency.

We have developed a room temperature tilted target technique to control the nanoparticle size and inter-particle distance in the sub-nm regime. With precision control of size and density, this work has branched out for novel applications in numerous fields $[1,2,4,5,8-11,25,26,51,53-58]$. We focus on using multiple layers of Pt nanoparticle for controlled layer by layer charge storage in NVM flash devices and the use of these NVM flash architectures combined with special neutron converter layer for demonstrating a highly efficient neutron detection which offer many potential advantages over the current state-of-the-art, including low cost and compactness. 


\subsection{Scope of the Dissertation}

This report is divided into 5 chapters dealing with all the aforementioned findings. Chapter 2 describes the effect of various deposition parameters on Pt nanoparticle growth. The importance of each deposition parameter is studied and their respective effect on the nanoparticle growth has been reported. The growth of these nanoparticles in the sub-2nm regime is discussed extensively based on High Resolution Transmission Electron Microscopy (HRTEM) images. Modelling of nanoparticle size with respect to change in deposition time and their relation with crystallinity is also discussed. In addition to the reported observations in nanoparticle configuration, an in depth understanding in energy of the metal atom cluster in the sputtering deposition plume allows us to tune the size and density of nanoparticles at will.

Chapter 3 describes the application of Pt nanoparticles developed in chapter 2 for charge storage applications. We focus in layer by layer charging for dual layer Pt NP embedded in $\mathrm{Al}_{2} \mathrm{O}_{3}$ dielectric. The effect of nanoparticle size, inter-particle distance, tunnelling oxide thickness and inter-particle-dielectric thickness has been studied. We also briefly describe the methods of characterization and techniques used to fabricate these novel architectures

Chapter 4 focuses on using devices similar to that used in chapter 3 for neutron detection. This chapter shows the study on the feasibility to fabricate MOSCAP NVM structures with a special neutron converter layer. The effect of different device structures has been verified for best device performance using converter layers as dielectrics. More

specifically, ${ }^{10} \mathrm{~B}$ enriched Polystyrene has been used as capping oxide layers for the NVM 
Flash devices. In addition, this chapter gives the necessary background work for a very promising- future research concept for sensitive neutron detection.

This report concludes with chapter 5, where the major conclusions have been derived and the plans for future work has been discussed. 


\subsection{References}

[1] J. R. C. Jeff, M. Yun, B. Ramalingam, B. Lee, V. Misra, G. Triplett, and S. Gangopadhyay, "Charge storage characteristics of ultra-small Pt nanoparticle embedded GaAs based non-volatile memory," Applied Physics Letters, vol. 99, pp. 072104-3, 2011.

[2] M.Yun, B.Ramalingam, H.Zheng, and S.Gangopadhyay, "Controllable Memory Window Behavior of Size Tunable Pt Nanoparticle Embedded Organic Based Nonvolatile Memory Transistors," MRS Online Proceedings Library, vol. 1359, 2011.

[3] Y. Minseong, D. W. Mueller, M. Hossain, V. Misra, and S. Gangopadhyay, "Sub2 nm Size-Tunable High-Density Pt Nanoparticle Embedded Nonvolatile Memory," Electron Device Letters, IEEE, vol. 30, pp. 1362-1364, 2009.

[4] M. Yun, B. Ramalingam, and S. Gangopadhyay, "Room temperature observation of size dependent single electron tunneling in a sub-2 $\mathrm{nm}$ size tunable $\mathrm{Pt}$ nanoparticle embedded metal-oxide-semiconductor structure," Nanotechnology, vol. 22, p. 465201, 2011.

[5] M. Yun, B. Ramalingam, and S. Gangopadhyay, "Multi-Layer Pt Nanoparticle Embedded High Density Non-Volatile Memory Devices," Journal of The Electrochemical Society, vol. 159, pp. H393-H399, 2012.

[6] J. Dufourcq, S. Bodnar, G. Gay, D. Lafond, P. Mur, G. Molas, J. P. Nieto, L. Vandroux, L. Jodin, F. Gustavo, and T. Baron, "High density platinum nanocrystals for non-volatile memory applications," Applied Physics Letters, vol. 92, pp. 073102-3, 2008. 
[7] J. Dufourcq, P. Mur, M. J. Gordon, S. Minoret, R. Coppard, and T. Baron, "Metallic nano-crystals for flash memories," Materials Science and Engineering: C, vol. 27, pp. 1496-1499, 2007.

[8] S. Mukherjee, B. Ramalingam, L. Griggs, S. Hamm, G. A. Baker, P. Fraundorf, S. Sengupta, and S. Gangopadhyay, "Ultrafine sputter-deposited Pt nanoparticles for triiodide reduction in dye-sensitized solar cells: impact of nanoparticle size, crystallinity and surface coverage on catalytic activity," Nanotechnology, p. In Print, 2012.

[9] W.-N. Wang, W.-J. An, B. Ramalingam, S. Mukherjee, D. M. Niedzwiedzki, S. Gangopadhyay, and P. Biswas, "Size and Structure Matter: Enhanced CO2 Photoreduction Efficiency by Size-Resolved Ultrafine Pt Nanoparticles on TiO2 Single Crystals," Journal of the American Chemical Society, vol. 134, pp. 1127611281, 2012/07/11 2012.

[10] W.-J. An, W.-N. Wang, B. Ramalingam, S. Mukherjee, B. Daubayev, S. Gangopadhyay, and P. Biswas, "Role of Pt Nanoparticles in Photoreactions on TiO2 Photoelectrodes," MRS spring meeting and Exhibit, 2012.

[11] W.-N. Wang, W.-J. An, B. Ramalingam, S. Mukherjee, D. M. Niedzwiedzki, S. Gangopadhyay, and P. Biswas, "Size and Structure Matter: Enhanced CO2 Photoreduction Efficiency by Size-resolved Ultrafine Pt Nanoparticles on TiO2 Single Crystals," Journal of the American Chemical Society, 2012.

[12] H.-T. Lue, K.-Y. Hsieh, and C.-Y. Lu, "Overview of Advanced 3D Charge-trapping Flash Memory Devices," MRS Online Proceedings Library, vol. 1250, pp. nullnull, 2010. 
[13] S. Tiwari, F. Rana, H. Hanafi, A. Hartstein, E. F. Crabbe, and K. Chan, "A silicon nanocrystals based memory," Applied Physics Letters, vol. 68, pp. 1377-1379, 1996.

[14] H. I. Hanafi, S. Tiwari, and I. Khan, "Fast and long retention-time nano-crystal memory," Electron Devices, IEEE Transactions on, vol. 43, pp. 1553-1558, 1996.

[15] S. Tiwari, F. Rana, K. Chan, L. Shi, and H. Hanafi, "Single charge and confinement effects in nano-crystal memories," Applied Physics Letters, vol. 69, pp. 1232-1234, 1996.

[16] K. Ya-Chin, K. Tsu-Jae, and H. Chenming, "Charge-trap memory device fabricated by oxidation of $\mathrm{Si}<\mathrm{sub}>1-\mathrm{x}</ \mathrm{sub}>\mathrm{Ge}<\mathrm{sub}>\mathrm{x}</ \mathrm{sub}>$," Electron Devices, IEEE Transactions on, vol. 48, pp. 696-700, 2001.

[17] Z. Liu, C. Lee, V. Narayanan, G. Pei, and E. C. Kan, "Metal nanocrystal memories. I. Device design and fabrication," Electron Devices, IEEE Transactions on, vol. 49, pp. 1606-1613, 2002.

[18] Z. Liu, C. Lee, V. Narayanan, G. Pei, and E. C. Kan, "Metal nanocrystal memoriespart II: electrical characteristics," Electron Devices, IEEE Transactions on, vol. 49, pp. 1614-1622, 2002.

[19] J.-Y. Tseng, C.-W. Cheng, S.-Y. Wang, T.-B. Wu, K.-Y. Hsieh, and R. Liu, "Memory characteristics of Pt nanocrystals self-assembled from reduction of an embedded $\mathrm{PtO}[$ sub x] ultrathin film in metal-oxide-semiconductor structures," Applied Physics Letters, vol. 85, pp. 2595-2597, 2004.

[20] C.-C. Wang, Y.-K. Chiou, C.-H. Chang, J.-Y. Tseng, L.-J. Wu, C.-Y. Chen, and T.B. Wu, "Memory characteristics of Au nanocrystals embedded in metal-oxide- 
semiconductor structure by using atomic-layer-deposited $\mathrm{Al} 2 \mathrm{O} 3$ as control oxide," Journal of Physics D: Applied Physics, vol. 40, p. 1673, 2007.

[21] T. Z. Lu, M. Alexe, R. Scholz, V. Talelaev, and M. Zacharias, "Multilevel charge storage in silicon nanocrystal multilayers," Applied Physics Letters, vol. 87, p. 202110, 2005.

[22] A. Morales-Sánchez, J. Barreto, C. Domínguez, M. Aceves, Z. Yu, and J. A. LunaLópez, "Coulomb blockade effects in silicon nanoparticles embedded in thin silicon-rich oxide films," Nanotechnology, vol. 19, p. 165401, 2008.

[23] H. Tsuji, N. Arai, T. Matsumoto, K. Ueno, Y. Gotoh, K. Adachi, H. Kotaki, and J. Ishikawa, "Silver nanoparticle formation in thin oxide layer on silicon by silvernegative-ion implantation for Coulomb blockade at room temperature," Applied Surface Science, vol. 238, pp. 132-137, 2004.

[24] J. Herrmann, D. J. Bray, K. H. Müller, G. Wei, and L. F. Lindoy, "Tuning the Coulomb charging energy in cross-linked nanoparticle films," Physical Review B, vol. 76, p. 212201, 2007.

[25] M.Yun, H.Zheng, B.Ramalingam, and S. Gangopadhyay, "Controllable memory window behavior of size tunable Pt nanoparticle embedded pentacene based nonvolatile memory transistor," Electrochemical Society, 2013.

[26] B. Ramalingam, H.Zheng, and S. Gangopadhyay, "Multi-Bit Operation in NonVolatile Memory Devices using Controlled Charging Behavior for Double-Layer Floating Gate Devices," under preparation, 2013. 
[27] X. R. Ye, Y. Lin, C. Wang, and C. M. Wai, "Supercritical fluid fabrication of metal nanowires and nanorods templated by multiwalled carbon nanotubes," Advanced Materials, vol. 15, pp. 316-319, 2003.

[28] G. G. Wildgoose, C. E. Banks, and R. G. Compton, "Metal nanoparticles and related materials supported on carbon nanotubes: methods and applications," Small, vol. 2, pp. 182-193, 2006.

[29] Z. Liu, L. M. Gan, L. Hong, W. Chen, and J. Y. Lee, "Carbon-supported Pt nanoparticles as catalysts for proton exchange membrane fuel cells," Journal of Power Sources, vol. 139, pp. 73-78, 2005.

[30] T. Matsumoto, T. Komatsu, H. Nakano, K. Arai, Y. Nagashima, E. Yoo, T. Yamazaki, M. Kijima, H. Shimizu, and Y. Takasawa, "Efficient usage of highly dispersed Pt on carbon nanotubes for electrode catalysts of polymer electrolyte fuel cells," Catalysis Today, vol. 90, pp. 277-281, 2004.

[31] M. Zhao and R. M. Crooks, "Homogeneous hydrogenation catalysis with monodisperse, dendrimer-encapsulated Pd and Pt nanoparticles," Angewandte Chemie International Edition, vol. 38, pp. 364-366, 1999.

[32] Y. Xing, "Synthesis and electrochemical characterization of uniformly-dispersed high loading Pt nanoparticles on sonochemically-treated carbon nanotubes," The Journal of Physical Chemistry B, vol. 108, pp. 19255-19259, 2004.

[33] C. Paoletti, A. Cemmi, L. Giorgi, R. Giorgi, L. Pilloni, E. Serra, and M. Pasquali, "Electro-deposition on carbon black and carbon nanotubes of Pt nanostructured catalysts for methanol oxidation," Journal of Power Sources, vol. 183, pp. 84-91, 2008. 
[34] J. Zhang, B. P. Ting, M. Khan, M. C. Pearce, Y. Yang, Z. Gao, and J. Y. Ying, "Pt nanoparticle label-mediated deposition of $\mathrm{Pt}$ catalyst for ultrasensitive electrochemical immunosensors," Biosensors and Bioelectronics, vol. 26, pp. 418423, 2010.

[35] M. Yang, Y. Yang, Y. Liu, G. Shen, and R. Yu, "Platinum nanoparticles-doped solgel/carbon nanotubes composite electrochemical sensors and biosensors," Biosensors and Bioelectronics, vol. 21, pp. 1125-1131, 2006.

[36] A. Chatterjee and D. Chakravorty, "Electrical conductivity of sol-gel derived metal nanoparticles," Journal of materials science, vol. 27, pp. 4115-4119, 1992.

[37] L. Qu and L. Dai, "Substrate-enhanced electroless deposition of metal nanoparticles on carbon nanotubes," Journal of the American Chemical Society, vol. 127, pp. 10806-10807, 2005.

[38] L. A. Porter, H. C. Choi, A. E. Ribbe, and J. M. Buriak, "Controlled electroless deposition of noble metal nanoparticle films on germanium surfaces," Nano Letters, vol. 2, pp. 1067-1071, 2002.

[39] P. Calandra, G. Calogero, A. Sinopoli, and P. G. Gucciardi, "Metal Nanoparticles and Carbon-Based Nanostructures as Advanced Materials for Cathode Application in Dye-Sensitized Solar Cells," International Journal of Photoenergy, vol. 2010, 2010.

[40] M. Hirasawa, H. Shirakawa, H. Hamamura, Y. Egashira, and H. Komiyama, "Growth mechanism of nanoparticles prepared by radio frequency sputtering," Journal of Applied Physics, vol. 82, pp. 1404-1407, 1997. 
[41] J. J. Brege, C. E. Hamilton, C. A. Crouse, and A. R. Barron, "Ultrasmall Copper Nanoparticles from a Hydrophobically Immobilized Surfactant Template," Nano Letters, vol. 9, pp. 2239-2242, 2009.

[42] A. R. Canário, E. A. Sanchez, Y. Bandurin, and V. A. Esaulov, "Growth of Ag nanostructures on TiO2(1 1 0)," Surface Science, vol. 547, pp. L887-L894, 2003.

[43] J. Fu and Y. Zhao, "Au nanoparticle based localized surface plasmon resonance substrates fabricated by dynamic shadowing growth," Nanotechnology, vol. 21, p. $175303,2010$.

[44] S. P. Park, S. S. Kim, J. H. Kim, C. N. Whang, and S. Im, "Optical and luminescence characteristics of thermally evaporated pentacene films on $\mathrm{Si}$," Applied Physics Letters, vol. 80, pp. 2872-2874, 2002.

[45] Z.-Q. Tian, B. Ren, and D.-Y. Wu, "Surface-Enhanced Raman Scattering: From Noble to Transition Metals and from Rough Surfaces to Ordered Nanostructures," The Journal of Physical Chemistry B, vol. 106, pp. 9463-9483, 2002.

[46] Y. Volokitin, J. Sinzig, L. J. de Jongh, G. Schmid, M. N. Vargaftik, and I. I. Moiseevi, "Quantum-size effects in the thermodynamic properties of metallic nanoparticles," Nature, vol. 384, pp. 621-623, 1996.

[47] R. Yu, H. Song, X.-F. Zhang, and P. Yang, "Thermal Wetting of Platinum Nanocrystals on Silica Surface," The Journal of Physical Chemistry B, vol. 109, pp. 6940-6943, 2005.

[48] J. Zuo, "Deposition of Ag nanostructures on $\mathrm{TiO} 2$ thin films by RF magnetron sputtering," Applied Surface Science, vol. 256, pp. 7096-7101, 2010. 
[49] P. Brault, A.-L. Thomann, and C. Andreazza-Vignolle, "Percolative growth of palladium ultrathin films deposited by plasma sputtering," Surface Science, vol. 406, pp. L597-L602, 1998.

[50] W.-J. An, W.-N. Wang, B. Ramalingam, S. Mukherjee, B. Daubayev, S. Gangopadhyay, and P. Biswas, "Enhanced Water Photolysis with Pt Metal Nanoparticles on Single Crystal TiO2 Surfaces," Langmuir, vol. 28, pp. 7528-7534, 2012/05/15 2012.

[51] Y. Minseong, R. Balavinayagam, and G. Shubhra, "Room temperature observation of size dependent single electron tunneling in a sub- $2 \mathrm{~nm}$ size tunable $\mathrm{Pt}$ nanoparticle embedded metal-oxide-semiconductor structure," Nanotechnology, vol. 22, p. 465201, 2011.

[52] B. E. Roustom, G. Fóti, and C. Comninellis, "Preparation of gold nanoparticles by heat treatment of sputter deposited gold on boron-doped diamond film electrode," Electrochemistry Communications, vol. 7, pp. 398-405, 2005.

[53] H. Zheng, R. Balavinayagam, V. Korampally, and G. Shubhra, "Large sensitivity enhancement in semiconducting organic field effect transistor sensors through incorporation of ultra-fine Platinum nanoclusters," Applied Physics Letters, vol. submitted, 2013.

[54] B. Ramalingam, S. Mukherjee, C. J. Mathai, K. Gangopadhyay, and S. Gangopadhyay, "Sub-2 $\mathrm{nm}$ size and density tunable platinum nanoparticles using room temperature tilted-target sputtering," Nanotechnology, vol. 24, p. 205602, 2013. 
[55] H.Zheng, B.Ramalingam, V. korampally, and S. Gangopadhyay, "In-situ Trace Vapor Explosives Detection Using Pentacene Thin-Film-Transistor with Embedded Sub-1nm Platinum Nanoparticles " vol. under preparation, 2013.

[56] B.Ramalingam, M. I. Lee, S. Kovaleski, K. Gangopadhyay, and S. Gangopadhyay, "Advance Boron compound dielectrics for trace neutron detection," Under preparation, 2013.

[57] B. Ramalingam, S. Mukherjee, C. Mathai, K. Gangopadhyay, and S. Gangopadhyay, "Sub-2 nm Size and Density Tuneable Platinum Nanoparticles using Room Temperature Tilt-Target Sputtering " Nanotechnology, vol. Accepted , in press, submitted Feb 20132013.

[58] M. Somik, R. Balavinayagam, G. Lauren, H. Steven, A. B. Gary, F. Phil, S. Shramik, and G. Shubhra, "Ultrafine sputter-deposited Pt nanoparticles for triiodide reduction in dye-sensitized solar cells: impact of nanoparticle size, crystallinity and surface coverage on catalytic activity," Nanotechnology, vol. 23, p. 485405, 2012. 


\section{CHAPTER 2: Sub-2 nm Platinum Nanoparticle Growth Study}

This chapter focuses on providing experimental data supporting several theories that have been proposed to explain the growth of sub-2 $\mathrm{nm}$ platinum nanoparticles. Data is also presented regarding size and density characteristics for different energetic regimes of deposition, and discussion follows regarding how this data helps in elucidating the precision control over size and inter-particle distance for these nanoparticles.

\subsection{Introduction}

Historically, metal nanoparticles have attracted strong interest in applications over various fields of study. Compared to semiconductor nanoparticles, metals have many advantages because of their larger work function, higher density of states at Fermi energy and their ability to tune the Schottky barrier height. Traditionally, the chemical synthesis route (bottom-up process) has been utilized for preparation of such metal nanoparticles with controlled sizes and size distributions [16, 17]. However, incorporating these nanoparticles in systems requiring subsequent top-down processing steps are not straightforward and may not be compatible with semiconductor device manufacturing. Another major drawback of chemical synthesis of these nanoparticles is the presence of undesirable side-products (impurities and capping agents), which may lead to surface poisoning, adversely affecting sustained catalysis. [18]

To address these issues, small nanoparticles have been synthesized using top-down techniques such as physical vapour deposition (PVD) and chemical vapor deposition 
(CVD). However, the large variation in the nanoparticle size and number density as observed in these studies is not desirable for applications focusing on size-dependent behavior [1, 15, 19-25]. Top-down approaches like dewetting [14, 23, 26] and direct deposition $[19,21,27,28]$ have also been used to create nanoparticles through the physical vapor deposition route. The dewetting technique is the most common and produces high density metallic nanoparticles but has limited control over their size, distribution, and number density due to random ripening at nucleation sites. Moreover, the required annealing step makes this technique incompatible with procedures requiring room temperature processes.

Overall, the limited control over the size, distribution and number density of the particles leads to issues with experimental reproducibility and failure to accurately ascertain specific size dependent properties. This is particularly crucial in the $0.5 \mathrm{~nm}-2$ $\mathrm{nm}$ nanoparticle size regime where Pt nanoparticles have been known to undergo a transformation in crystal structure, which also changes their catalytic and electronic properties $[9,11,13,29-32]$. Thus, it is important to identify techniques that result in better control over nanoparticle growth in terms of its size and distribution as well as its subsequent characteristics. This need to better control nanoparticle growth led us towards research, development and understanding of homogenously dispersed sub-2 nm metal nanoparticles which can be accurately controlled in the $0.5 \mathrm{~nm}-2 \mathrm{~nm}$ range in-terms of size and number density. This chapter describes and explains the sub $2 \mathrm{~nm}$ platinum nanoparticle growth in room temperature using a unique tilt target sputter configuration. This chapter describes the work that led to fabricating and understanding the growth of 
sub-nm Pt nanoparticle using the most sophisticated physical vapour deposition process: Sputtering.

\subsubsection{Metal nanoparticles using sputtering}

Among PVD processes, the sputtering technique is the most promising method due to its inherent controllability of many process parameters and its ability to generate a high number density of metal nanoparticles on the substrate surface (as high as $5 \times 10^{12}$ particles/cm $\left.{ }^{2}\right)[9,11,13,19,29-32]$. In a sputtering technique, potentially any metal can be deposited in a highly controllable manner. This is especially true in radio frequency (RF) sputtering, which can even perform at low operating pressure with minimum pressure conditions as low as 0.5 mTorr. Since in a sputtering deposition, the ejection of an atom from a solid target is purely a result of the bombarding energetic particles - controlling the energy of these particles can enable us to control the growth parameters of the deposited thin film. Typically, these energetic particles are characteristics of the type of plasma created - hence a precise control over the plasma conditions can help us achieve this control. The way to approach this would be controlling the chamber pressure and the plasma power applied at the cathode and anode. These parameters can define the population of the metal atoms that sputter out from the solid target and take part in the deposition process, but even this control is limited, and it often narrows down to a lowest value of pressure and power possible - so low that sustaining the plasma becomes a challenge below that value.

Researchers have generally utilized sputtering process at a low pressure $(<5$ mTorr $)$ and low power $(<40 \mathrm{~W})$ to obtain small particles in the $2-5 \mathrm{~nm}$ regime $[19,28]$. However, most of the work has been focused on placing the substrates directly in the main flux of 
sputtered atoms where a high density of metal atoms and clusters take part in the nanoparticle formation, thereby restricting the precision in size control. Typically, particle sizes are larger than $2 \mathrm{~nm}$ for such techniques due to the fast diffusion and coalescence of metal atoms on the substrate surface [19, 33]. Although sputter grown sub-2 nm Pt nanoparticles have been reported through sputtering on nitrogen doped carbon nanotubes [34], the lowest reported particle size is $\sim 1.5 \mathrm{~nm}$. But here the size dispersion includes nanoparticles which are larger than $3 \mathrm{~nm}$, making application of size-dependent behavior difficult.

There is more to manipulating metal atoms than size control utilizing chamber pressure and plasma power. The only possible technique left to modify in-flight metal atoms' density and energy is to take advantage of spatial variations where the path of the sputtered atom can itself modify its own energy. All sputtered particles leaving the target can collide with each other or other gas atoms during the flight towards the substrate. This collision alters the energy of the arriving particles in addition to their direction and momentum (a phenomenon more commonly called thermalization), which in turn modify the morphology and structure of the deposited thin film. Identifying these techniques can lead to precision control over nanoparticle growth.

\subsection{Substrate Surface Energy and Thin Film Growth Modes}

One has to note that nanoparticle growth is dependent on both the energy of arriving atoms and the energy of the substrate surface itself. Typically, in theory-for ideal surfaces - the surface energy is a dominating factor for type of layer growth on the substrate. In practical applications, the surface roughness with kinks, edges and step edges 
supersede this effect disturbing the ideal theoretical assumptions of thin film growth. To understand the effect of surface energy, it is best to understand its effect on ideal surfaces and then correlate it with practical applications. Typically, for ideal surfaces, depending on the difference in the energy of the substrate and the energy of the arriving atom, the growth modes vary.

Stranski

\section{Volmer Weber}

\section{Krastanov}

\section{Frank-van-der}
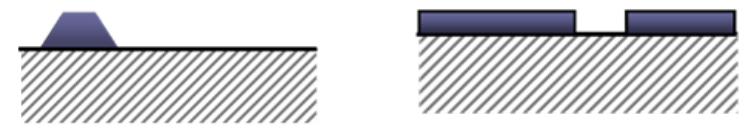

\section{Merwe}
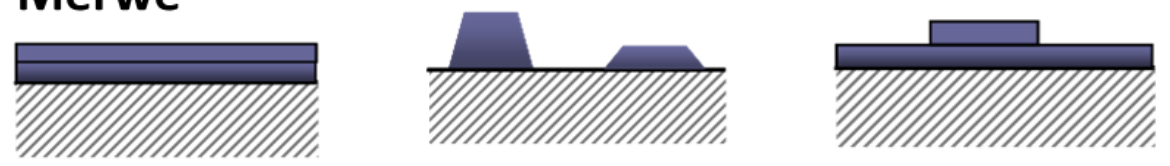

Figure 2-1: Three growth modes for thin film

Thin film growth models are classified in three growth modes[45]:

1. Layer by layer - Frank Van der Merwe - This growth occurs because atoms of deposit material are more strongly attracted to the substrate than they are to themselves. In this process, adatoms attach preferentially to surface sites resulting in atomically smooth, fully formed layers. This layer-by-layer growth is two dimensional, indicating that a continous film is formed prior to growth of subsequent layer. Figure 2-1 shows the layer by layer growth.

2. Island growth-Volmer Weber: In this growth mode, the scenario is somewhat contrary to Layer-by -Layer growth where the deposit atoms are more strongly 
bound to each other than they are to the substrate (Figure 2-1). Since the adatomadatom interactions are stronger than those of the adatom with the surface, this leads to the formation of three-dimensional adatom clusters or islands. Growth of these clusters, along with coarsening, will cause rough multi-layer films to grow on the substrate surface.

3. Layer + Island -Stranski Krastanov - This is a more common growth mode where layers form first and then, for one reason or another, the system switches to an island formation. Also known as 'layer-plus-island growth', the SK mode follows a two-step process: initially, complete films of adsorbates, up to several monolayers thick, grow in a layer-by-layer fashion on a crystal substrate. Beyond a critical layer thickness, which depends on strain and the chemical potential of the deposited film, growth continues through the nucleation and coalescence of adsorbate 'islands'.

If $\Upsilon$ represents surface tension and we deposit material A on B: We get layer growth if $\Upsilon_{a}<\Upsilon_{b}+\Upsilon^{*}$ and vice versa for Island growth. Here, in this equation $\Upsilon^{*}$ is the interface energy. The Stranski Krastanov (SK) mode arises when interface energy is more, and this usually happens when the layer thickness increases. In island growth mode, adatom concentration on the surface is small at the equilibrium vapor pressure point of deposit. No deposit will take place unless there is large super-saturation. In layer growth, the equilibrium vapor pressure is approached, thus all processes occur under saturation. As mentioned earlier, the above growth modes are for ideal surfaces; real surfaces always present some defects such as steps, vacancies or chemical impurities. The presence of these surface defects can alter the growth of metal films. 
Vapor deposition of metals leads to solid aggregate formation and then coalescence to form larger aggregates of the same form. If the temperature is not high, there will be no Brownian motion, and the motion will be purely attributed to surface properties (tension). For any deposition process, particle growth is strongly dependent on surface migration of adatoms and nanoparticles. For metal growth analysis, the migration of nanoparticles occurs only when the material target is sputtered. When precursors like metal atoms arrive at the surface, they bond (chemically) with particles releasing energy, i.e., bonding energy. Sometimes, this energy is enough to overcome the potential barrier for surface migration. This bonding energy is highly characteristic of the surface and can be controlled by controlling surface properties. Typically a low surface energy substrate will have lower bonding energy and vice-versa. Thus, any external energy from arriving clusters or from thermal energy greater than this bonding energy, can lead to lateral migration of deposited clusters at the substrate. One would like to control the atomic migration and coalescence at the substrate surface in order to form desired nanoparticles, adjusted in size and interparticle distance[19,41,45].

\subsubsection{Tilted Target Deposition}

To gain control over the nanoparticle formation process beyond the chamber pressure and plasma power limitation (using top down approaches), controlling the incoming metal/atom energy in the deposition profile is essential. Tilted target and glancing angle depositions (GLAD) have been used by researchers to modify this energy of the arrival atom/cluster for thin film growth, but none reported the study of nanoparticle formation and size control [35-37]. 
Typically, sputter deposition flux is comprised of high density metal atoms in the center and disperses out radially with lower density of metal atoms at the peripheral regions [8]. The generation of metal atoms with different energies in a radially varying metal atom flux density in the deposition flux is due to the dispersion of atoms in low pressure conditions inside the chamber by diffusion (Figure 2-2). Van Aeken et al. [38] performed Monte-Carlo simulations to simulate these deposition profiles based on factors like angle, radial arrangement of sample, and different target materials in $0.3 \mathrm{~Pa}-1 \mathrm{~Pa}$ range where a decrease in deposition rate due to the reduction in the atom flux density was observed as the sample moved out of the main deposition regime. The high metal atom flux density in the main deposition regime can increase the probability of in-flight collisions of metal atoms with other metal atoms or with process gas atoms, producing what is commonly known as thermalization [39-42]. Typically, a collision between two metal atoms will result in formation of clusters and a collision between metal and process gas atoms (e.g., Ar atoms) will result in an energy reduction of the atom thereby thermalizing it, which affects both nucleation and growth characteristics of nanoparticles as observed by Brault et al. [28] and Andreazza et al. [42] when they deposited Pt nanoparticles at different argon gas pressures.

Apart from change in metal atom energy with tilted target deposition, the other advantage which causes tilted target sputtering to be preferred over direct sputtering for nanoparticle fabrication is enhanced atomic shadowing. Atomic shadowing and adatom diffusion are the most dominant reasons for homogenous size distributions. In sputtering, atomic shadowing can occur even when the flux is normal to the substrate. During initial stages of film formation, when thin films are deposited with low adatom mobility, i.e., high 
melting point materials deposited onto room temperature substrates, or conversely when high surface energy material is deposited on low surface energy substrate, nanoparticles are produced. The consequent stage supports atomic shadowing, which helps keep the vapor flux from reaching certain trenches in the substrate due to surface roughness. Due to increased height, they capture the incident atoms and other areas get shadowed. The atoms following this initial stage of deposition grow on these particles, thereby leading to uniform growth.

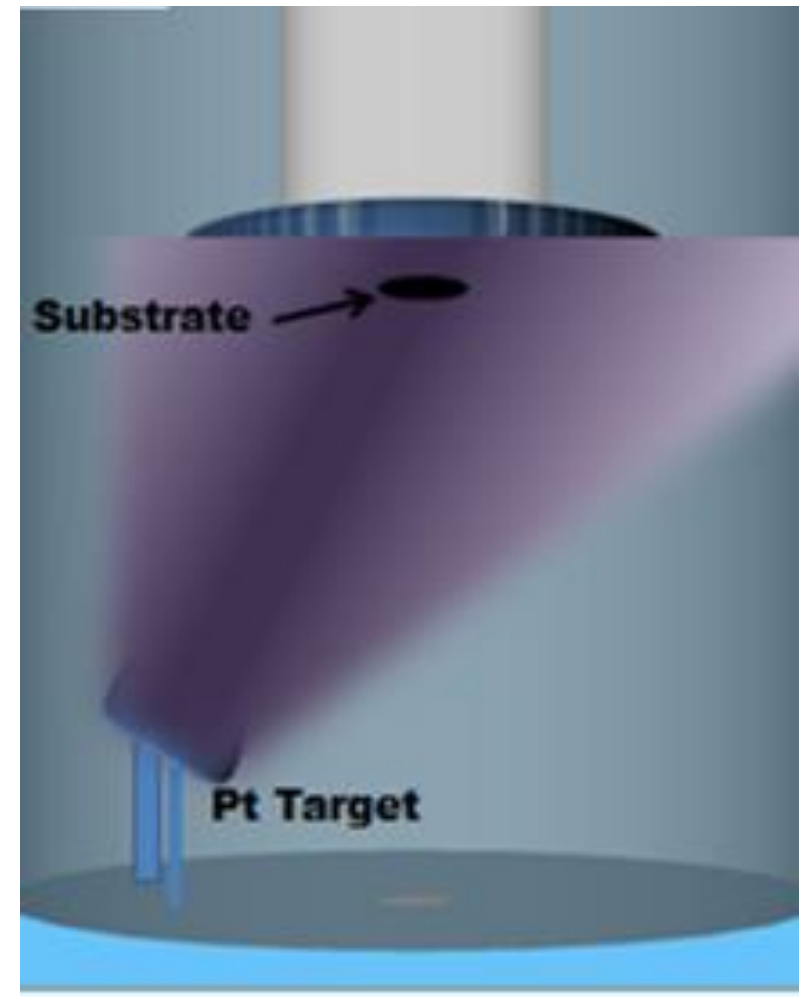

Figure 2-2: Representation of deposition plume for tilted target deposition 


\subsubsection{Rate equations and models}

In order to determine the type of growth, it is important to model experimental observations to match and understand theoretical assumptions. In metal aggregates growth and modeling, rate equations and models can describe the regimes of condensation and maximum cluster density. J. A. Venebles et al. have stated that conventional model calculations cannot be applied to match and explain experimental observations [41]. Any defect on the substrate surface overrules all other aspects and acts as preferred nucleation sites. Thus, most conventional rate equations assume perfect conditions of the substrate with a defect-free environment. Beysens et al. explained these experimental phenomena with a simple analogy using growth of water droplets. He hypothesized three successive growth stages.

1. Droplets nucleate and grow with no significant interactions between them (Fig 2-2 (a)). The droplet radius $<\mathrm{R}>$ increases according to power law:

$$
R \propto \tau^{x}
$$

where $\tau$-> $\quad \mathrm{t}^{*}(\mathrm{~F})^{1 / 2} \mathrm{t}$-time and F-flow rate(deposition rate)

2. When surface coverage becomes sufficiently large, the droplets interact by coalescence which makes them move (Figure 2-3 (b-c)).

3. At a time when the distance between droplets reach critical value, small droplets begin to be apparent between large droplets. The growth process reoccurs on these droplets giving us wide distributions (Figure 2-3 (d)). 


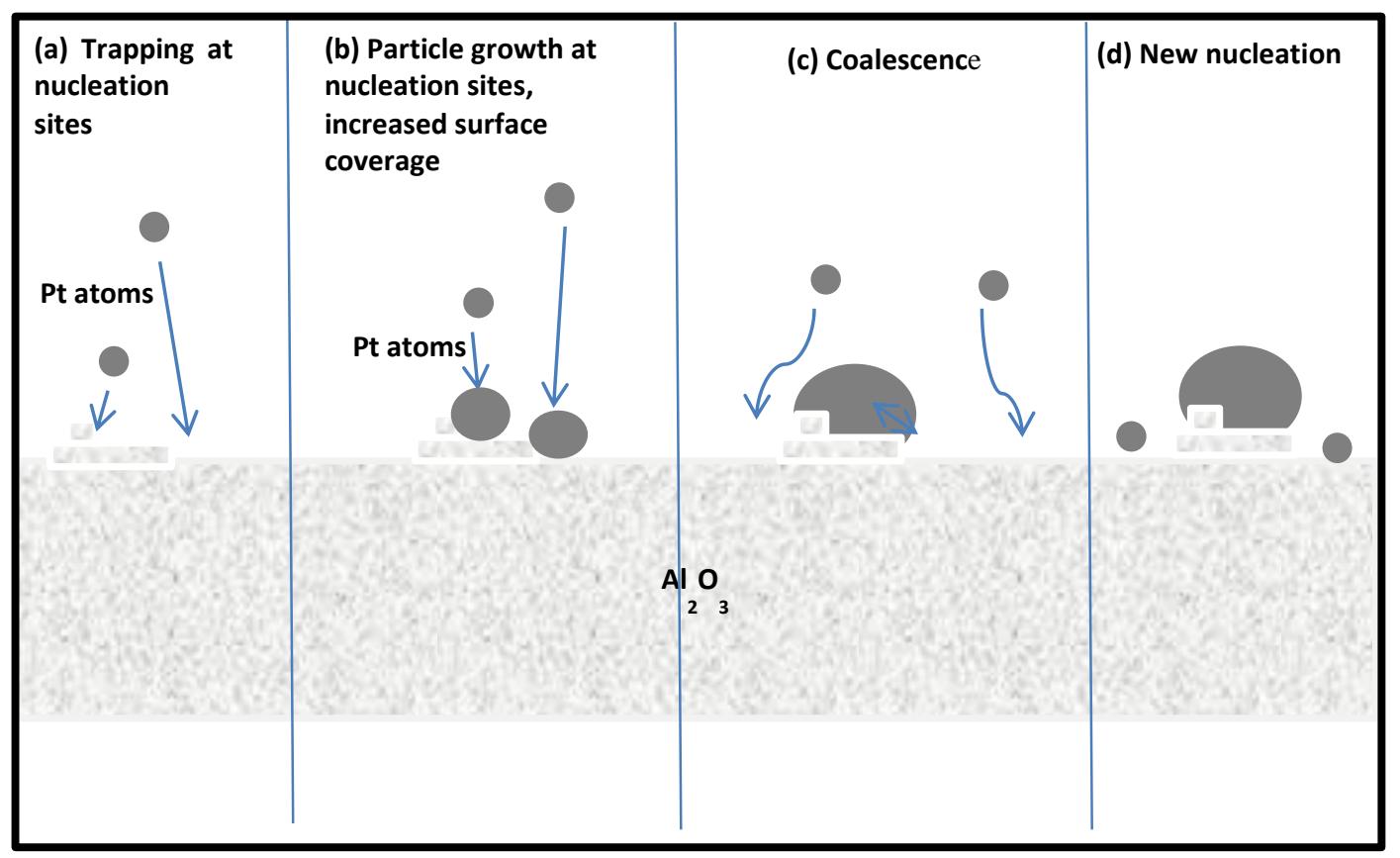

Figure 2-3: Particle growth phenomena demonstrating nucleation, growth, coalescence and new nucleation

Generally, on a smooth homogenous, isotropic substrate, the contact angle of a growing drop should be equal to that of a shrinking drop, technically, advancing and receding contact angles should be equal. But on an inhomogeneous substrate, defects distributed on a substrate provide surface roughness and act as physical obstacles for spreading of the drop. The border of the drop will stick as it moves across the surface giving the drop a larger contact angle as it grows and a smaller angle as it shrinks. Similar analogy has been used to understand the governing phenomena in growth of metal nanoparticles. Section 2.3.1 discusses how this thesis's rate equation can be used to determine the growth of metal nanoparticles on a surface. 


\subsection{Experiments}

\subsubsection{Sample Preparation}

An AJA International ATC 2000 magnetron sputtering system was used to deposit platinum nanoparticles (Figure 2-5). A two-inch platinum target with $99.99 \%$ purity from Kurt J. Lesker Corporation was mounted on a sputtering gun which can be tilted at an angle with respect to the substrate.

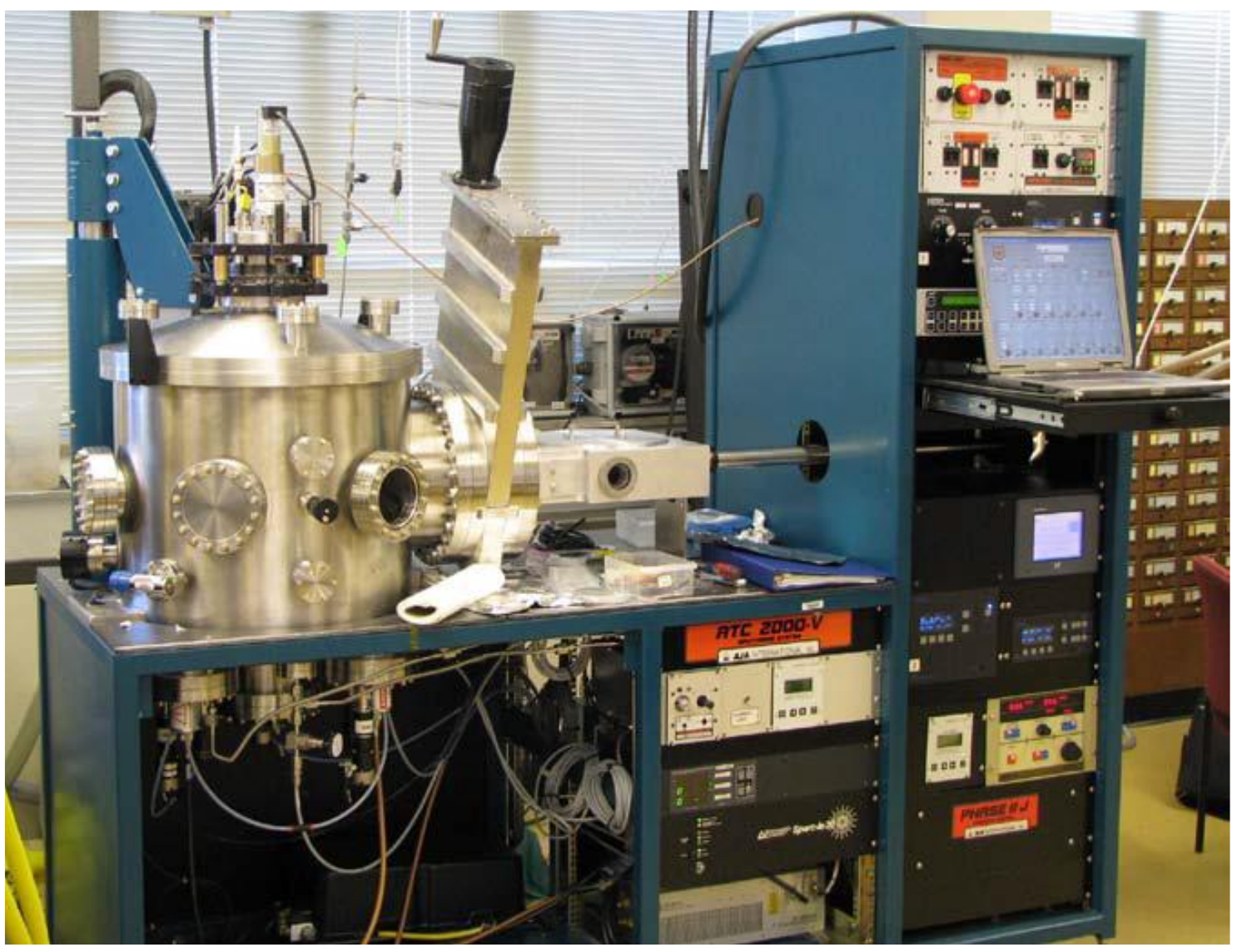

Figure 2-4: AJA Sputtering tool used for nanoparticle fabrication 
Prior to deposition, the chamber was evacuated to $10^{-8}$ Torr, and the platinum target was pre-sputtered for $600 \mathrm{~s}$ at $\mathrm{RF}$ deposition power of $100 \mathrm{~W}$ to clean the target. The substrate holder was configured as the anode (grounded). Platinum nanoparticles were deposited with a high purity Ar gas (99.999\%) with a flow rate $10 \mathrm{sccm}$ and a working pressure of 4 mTorr. The ambient temperature was $\sim 300 \mathrm{~K}$, with a six-inch target to substrate distance at $20 \mathrm{rpm}$ substrate rotation speed. An RF power $(13.56 \mathrm{MHz})$ varying from $20 \mathrm{~W}$ to $60 \mathrm{~W}$ was used to sputter Pt nanoparticles at different deposition times starting from $10 \mathrm{~s}$ to $60 \mathrm{~s}$.

Table 2-1 shows the different sputter parameters varied in this work for $\mathrm{Pt}$ nanoparticle growth studies. Figure 2-5 shows the 3-D schematic view of the sputter chamber set up, which was used for controlling angular deposition flux in TTS configuration where the focal area of the magnetron sputtering source was fine-tuned for Pt nanoparticle deposition. For most studies, the TEM grids were placed at the centre of the substrate holder to minimize the influence of their radial displacement on the observed nanoparticle characteristics. To study the effect of substrate location, two grids were placed at centre and edge of the substrate to observe size variation with change in target angle, keeping deposition time and power constant. 
Table 2-1: Different sputter parameters used for Pt nanoparticle growth

\begin{tabular}{lllllllllllll}
\hline Experimental Parameter & \multicolumn{1}{l}{ Parameter values } \\
\hline Time (s) & 5 & 10 & 15 & 20 & 25 & 30 & 35 & 40 & 45 & 50 & 55 \\
Power (W) & 20 & 24 & 27 & 30 & 40 & 50 & 60 & & & \\
Target Angle ( deg) & 16.2 & 23.8 & 38.8 & & & & \\
Pressure & 4 mTorr (constant) & & & \\
Argon Gas flow & 10 sccm (constant) \\
Deposition Temperature & 293 K (room temperature) \\
Substrate Height (inches) & $6 "$ (constant) \\
\hline
\end{tabular}



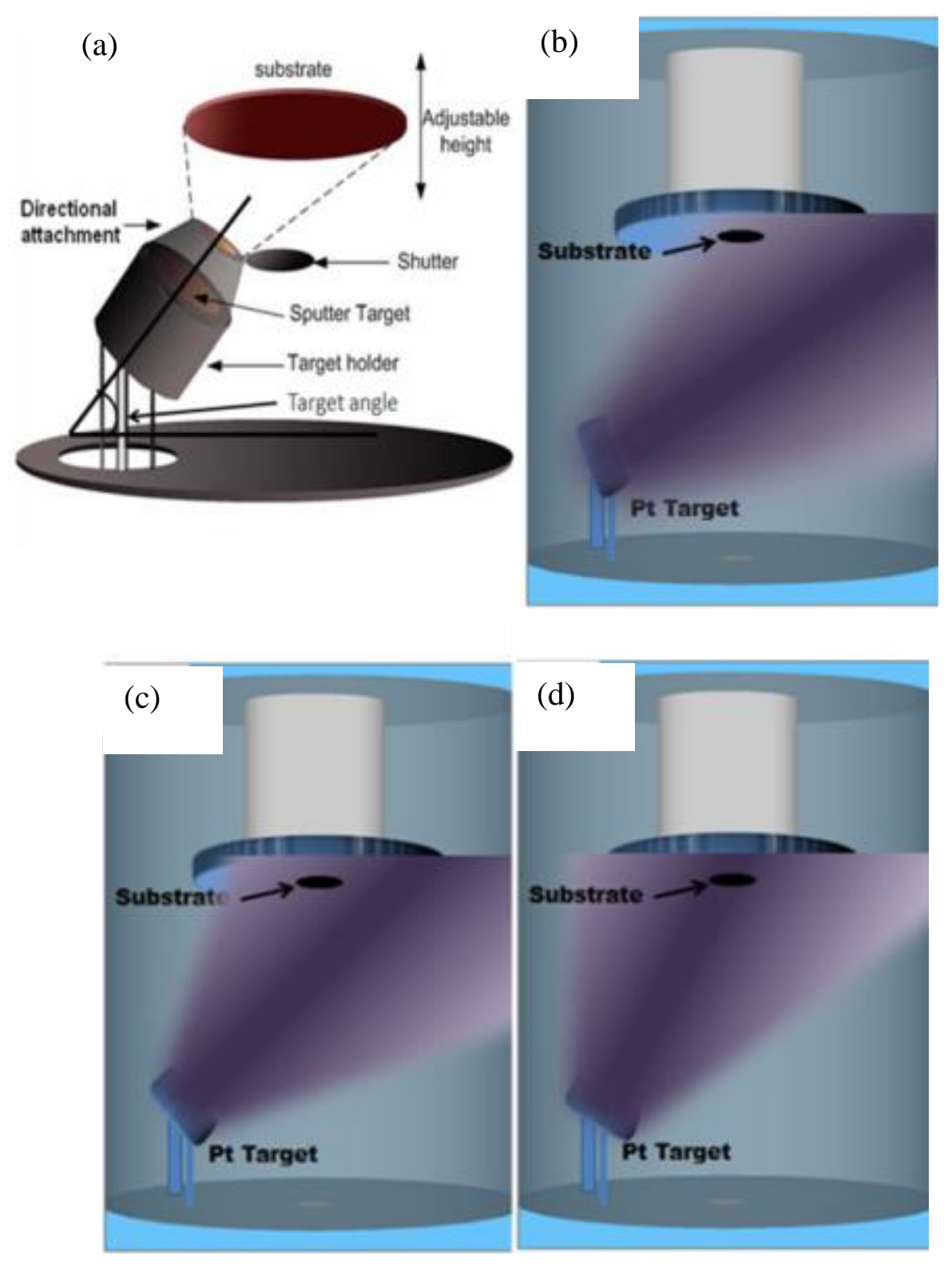

Figure 2-5: (a) Sputter chamber set-up with tilt-target configuration representing an interpretation of different spatial parameters in the sputtering chamber, which are involved in the nanoparticle growth process. The remaining images $(b-d)$ show atom flux with tilt-target configuration at (b) $16.2^{\circ}$ (c) $23.8^{\circ}$ and (d) $38.8^{\circ}$. 


\subsubsection{Sample Characterization}

The Platinum nanoparticles were characterized using a high resolution transmission electron microscopy (HRTEM), Technai F20 (200kV), with beam shift capabilities. TEM images were used to determine particle size, shape, and density using statistical image analysis software, SPIP 5.0 from Image Metrology A/S. Statistical data analysis was performed using software $\mathrm{R}^{\mathrm{TM}}[43]$.

For image characterization, a $5 \mathrm{~nm}$ of amorphous $\mathrm{Al}_{2} \mathrm{O}_{3}$ thin film was deposited on Holey-carbon film grid using a Kurt J. Lesker AXXIS electron beam evaporator at room temperature, details of which are available in [11]. Since roughness and surface energy of the $\mathrm{Al}_{2} \mathrm{O}_{3}$ can strongly influence nanoparticle growth characteristics and its crystallinity [41], the $\mathrm{Al}_{2} \mathrm{O}_{3}$ surface properties were characterized (atomic force microscopy and surface energy) and were left unchanged in all Pt nanoparticle growth experiments (Figure 2-6 and Table 2-2). The absolute value for the roughness of the e-beam grown $\mathrm{Al}_{2} \mathrm{O}_{3}$ is $0.22 \mathrm{~nm}$ (AFM details provided in (Figure 2-6 and Table 2-2) and the surface energy was measured to be 29.7 dynes/cm (using VCA optima contact angle measurement).

It is important to note that $\mathrm{Pt}$ nanoparticle crystal growth is directed by the growth process of sputtered Pt nanoparticles, and the nanoparticle growth process is governed by surface energy difference between the incoming Pt atom (surface energy $>2500$ dynes $/ \mathrm{cm}$ ) and the substrate $[19,44,45]$. 


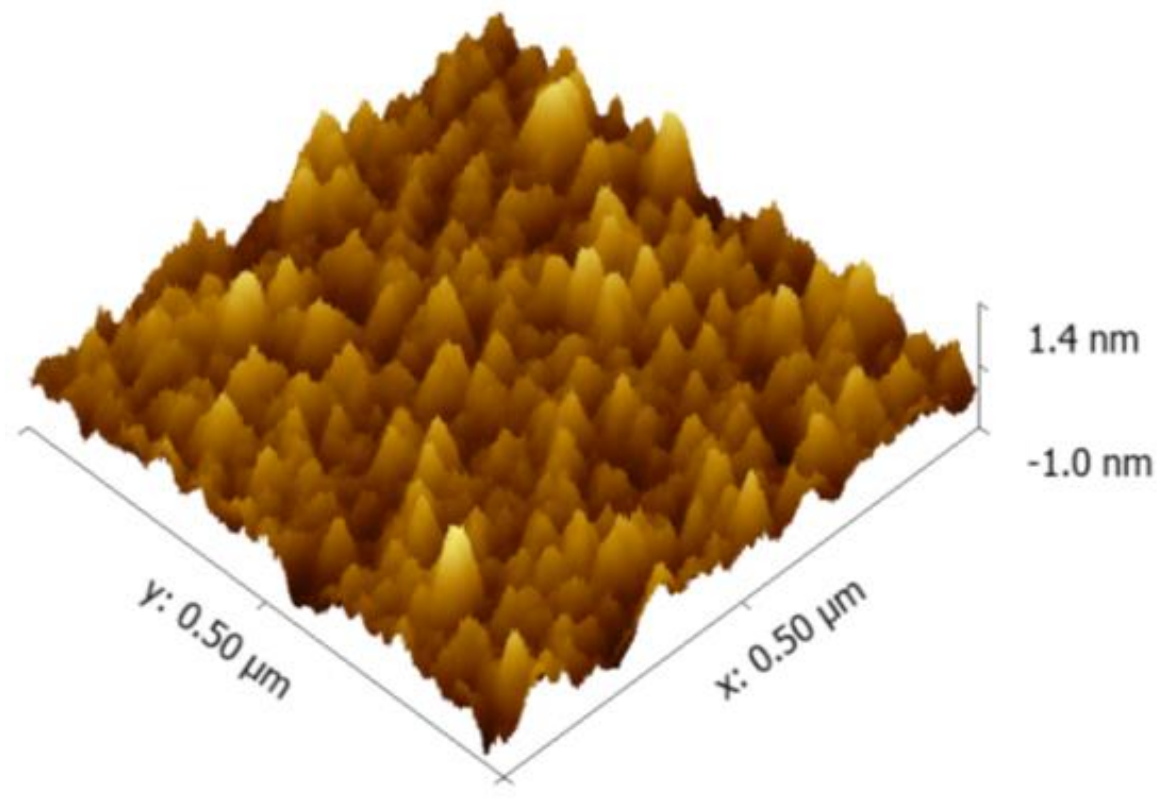

Figure 2-6: 3-D profile of $\mathrm{Al}_{2} \mathrm{O}_{3}$ surface roughness and inset table surface showing roughness characteristics measured using AFM.

Table 2-2: $\mathrm{Al}_{2} \mathrm{O}_{3}$ roughness parameter from $\mathrm{AFM}$ images

\begin{tabular}{ll}
\hline PARAMETER & VALUES \\
\hline Minimum & $-1.029 \mathrm{~nm}$ \\
Maximum & $1.359 \mathrm{~nm}$ \\
Median & $-0.016 \mathrm{~nm}$ \\
Ra & $0.219 \mathrm{~nm}$ \\
Rms & $0.279 \mathrm{~nm}$ \\
\hline
\end{tabular}


To minimize beam induced coalescence effects from HRTEM imaging, a $5 \mathrm{~nm}$ $\mathrm{Al}_{2} \mathrm{O}_{3}$ capping layer was deposited by electron-beam evaporation at room temperature on Pt nanoparticles. A special imaging procedure was implemented to avoid time dependent beam exposure induced effects, which was observed to be more pronounced for sub-1 nm particles. Due to the high energy incident on the sample, the Pt atoms on the surface gain enough energy to move on the substrate, thereby coalescing. Since this effect is due to the characterization technique and not a result of the growth process itself, the beam induced coalescence results in exaggerating the size and leads to underestimating the number density of the particle. To avoid this, the electron beam was focused and corrected for stigmation at one place, then shifted in $\mathrm{XY}$ direction by a $\sim 4$ micron $\times 4$ micron area. The image was immediately taken within an exposure time of less than $5 \mathrm{~s}$ to minimize electron beam irradiation effect. This shift does not cause physical movement of the sample, and thus sample drift was avoided. Since prolonged electron beam exposure would change the actual particle size and density, it is important to eliminate this effect by reducing the exposure time.

In order to identify effects of electron beam induced coalescence, HRTEM images of the particles were imaged at different times at the same grid location. Figure 2-7 (a) shows the image with less than $5 \mathrm{~s}$ beam exposure where there are six or seven ultra-fine (sub-1 nm) particles appearing. Figure 2-7 (b) shows a larger number and size of particles with longer beam exposure time. Although the nanoparticles were capped with $\mathrm{Al}_{2} \mathrm{O}_{3}$, the electron beam irradiation of the Pt nanoparticles accelerated the lateral coalescence process, which makes the ultra-small particles come together and form larger particles, making imaging easier. Even though longer beam exposure images show a larger number of $\mathrm{Pt}$ 
nanoparticles, the characterization of the actual size of the Pt nanoparticles is compromised due to beam induced coalescence. This technique is thus misleading and may not estimate the nanoparticle size and number density accurately, particularly for sub-1 $\mathrm{nm}$ particles. To avoid this coalescence process, the images for actual characterization purposes were taken with a minimum beam exposure time using a stigmation technique described above. Even after taking all precautions, it was difficult to image particles with sizes of $0.5 \mathrm{~nm}$ or less, resulting in underestimation of the densities and overestimation of the sizes. Since the characterization techniques for such small size nanoparticles are limited, the discussion of growth of the Pt nanoparticles with different sputtering parameters is based on the HRTEM results. 

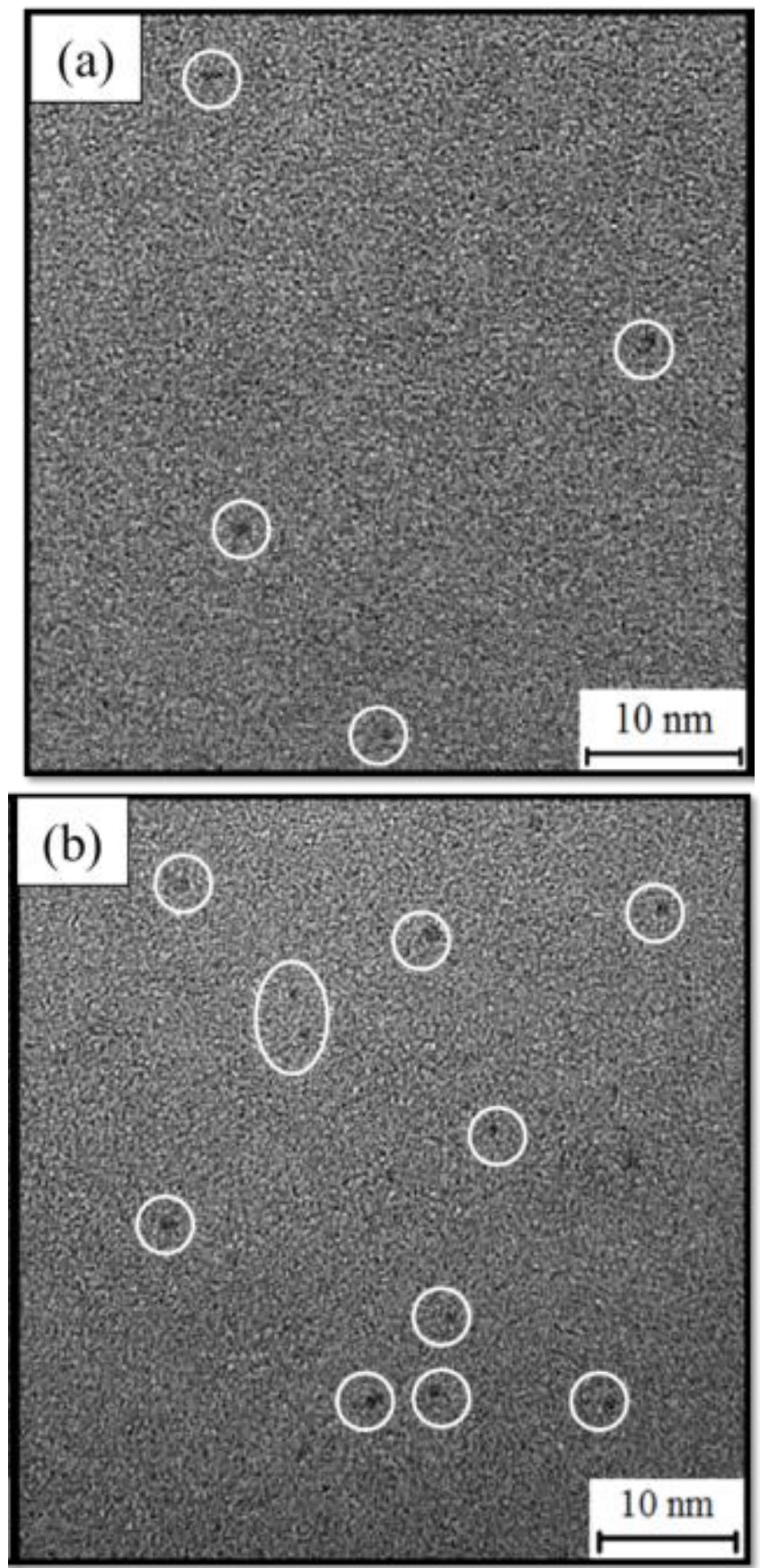

Figure 2-7: (a) HRTEM of Pt nanoparticles on $\mathrm{Al}_{2} \mathrm{O}_{3}$ with less than $5 \mathrm{~s}$ beam exposure and (b) with greater than $10 \mathrm{~s}$ beam exposure. (The white circles highlight the presence of extremely small Pt nanoparticles) 


\subsubsection{Image analysis}

Several iterations of experiments were performed to characterize the growth dynamics of the particles as a function of deposition time, power and tilt-target angle. The obtained TEM images were used to determine particle size, shape, and density using statistical image analysis software, SPIP 5.0, from Image Metrology A/S. The images and data are representative of several analysed images and applicable error bars are given to represent the distribution of size with image-to-image variation across different grid regions. Number of observations varied between $400-900$ particles per condition depending on the density of particles. The mean and standard deviation were calculated using the general formulas for arithmetic mean and standard deviation. Where mean is given by:

$$
\bar{x}=\frac{1}{n} \sum_{i=1}^{n} x_{i}
$$

And standard deviation, 's' is given by $(\mathrm{N}=$ sample size $)$

$$
s=\sqrt{\frac{1}{N-1} \sum_{i=1}^{N}\left(x_{i}-\bar{x}\right)^{2}} .
$$

Statistical data analysis was performed on these observations. For sample-tosample variation, the deposition condition with $30 \mathrm{~W}$ deposition power, $20 \mathrm{~s}$ deposition time and a 23.8 degree target angle was fabricated and analysed at least five times to find that the mean diameter was always within the $10 \%$ region of the error bar. For size dependent crystallinity verification, the samples were characterized using the same HRTEM. 


\subsection{Results and Discussions}

In previously published literature, the general consensus on the growth of sputtered metal particle on $\mathrm{Al}_{2} \mathrm{O}_{3}$ like surfaces has always been as follows: First, small clusters nucleate on the $\mathrm{Al}_{2} \mathrm{O}_{3}$ surface and grow into bigger nanoparticles as reported previously by Andreazza et al. [42]. This typically occurs when the cohesive energy (adatom-adatom) exceeds the adhesive energy (adatom-substrate atom). During deposition, when the energetic atoms strike the substrate, the released adatom-adatom binding energy $(\sim 1-2 \mathrm{eV})$ typically surpasses the adatom-substrate binding energy $(\sim 0.1 \mathrm{eV})$ leading to surface migration followed by lateral particle coalescence [42]. Throughout this study, three separate nanoparticle growth regimes were encountered (as shown in Figure 2-8):

1. First, in the nucleation governed growth regime, Pt atoms occupy the available nucleation sites without coalescence at the very early stages of nanoparticle growth. Thus, large number densities and small Pt cluster sizes with narrow size distribution can be obtained (Figure 2-8(a)).

2. Second, in the coalescence governed growth regime, Pt nanoparticles increase in size and number density occupying most of the nucleation sites, and the inter-atomic distance between these particles reduces. In this regime, relatively large size distributions may be encountered due to the coalescence and resultant availability of new nucleation sites. (Figure $2-8(b))$

3. Finally, in the agglomeration regime, the nanoparticles are big enough to undergo minimal surface diffusion and are relatively static leading to 
particle growth without coalescence. This third regime leads to the growth of nano-islands and eventually thin films. (Figure 2-8(c))

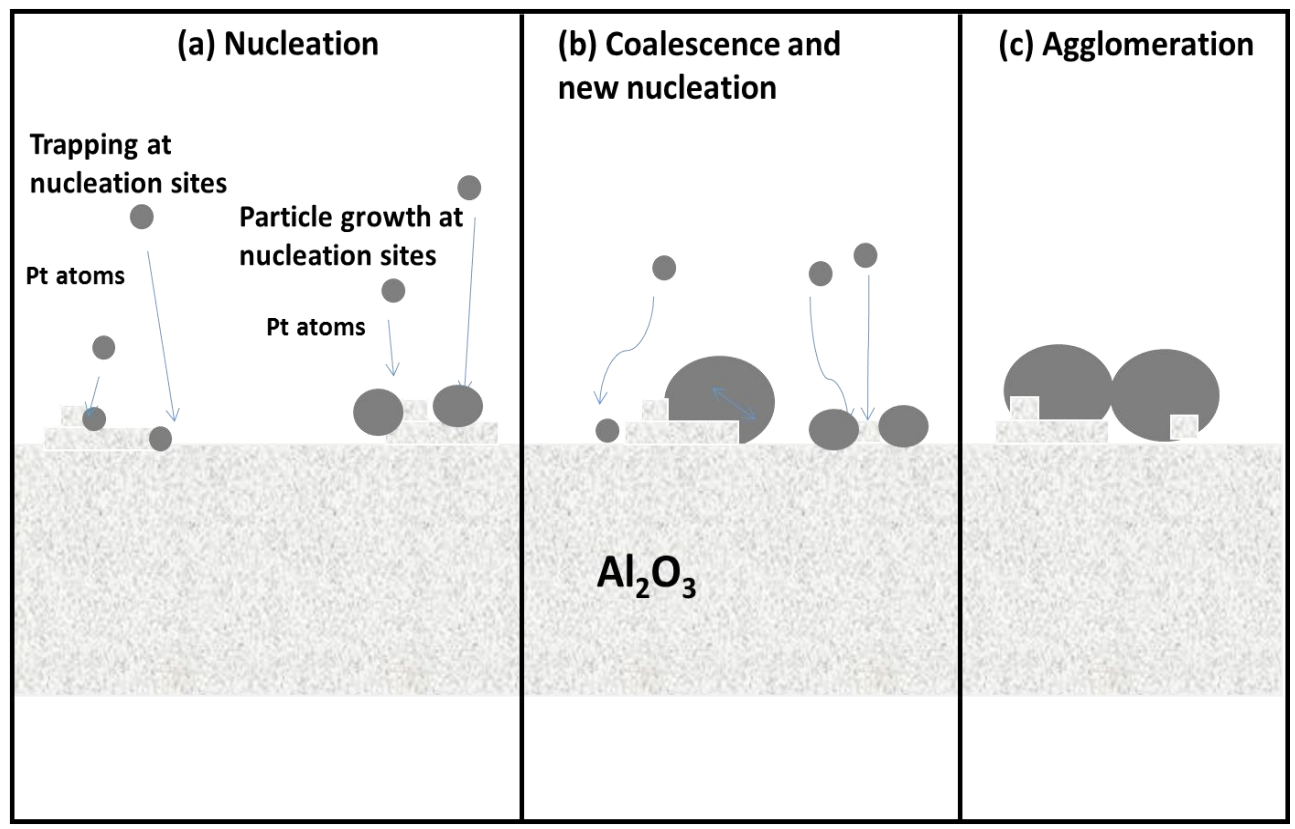

Figure 2-8: Schematic showing the different growth stages (a) Nucleation, (b) Coalescence and (c) Agglomeration 
The ability to control sub - nm Pt nanoparticle growth in the first regime is the highlight of this study, and we are not aware of any systematic study in published literature that reports growth characteristics of Pt clusters in this early nanoparticle growth regime. For sputtering time dependent nanoparticle growth, it was essential to identify nanoparticle 2-D and 3-D growth modes as well as different particle growth regimes, which are discussed in detail in Section 3.3.1. For Pt nanoparticle growth, it was recognized that thermalization of the metal atoms and their diffusion on the substrate surface are competing phenomenon, i.e., the higher the rate of thermalization within the sputtering chamber, the lower the energy of $\mathrm{Pt}$ atoms/clusters arriving at the surface and, therefore, a lower diffusion rate of the resultant Pt clusters/particles occurs on the surface. Discussion of nearly homogenous Pt nanoparticle distribution, an attribute essential for studying sizedependent nanoparticle behavior is presented in Section 3.3.2. While studying target angle dependence on Pt nanoparticle size and number density, the substrate exposure (or its lack of exposure) to the focal area of the deposition flux (Figure 2-5) was found to be of critical importance, the details of which are discussed in Section 3.3.3. A time-dependent analysis of nanoparticle growth at different target angles is presented in Section 3.4. Sputtering power dependence on Pt nanoparticle growth helped distinguish domains where thermalization overwhelms diffusion as the key factor associated with nanoparticle growth, and a thorough discussion of this effect is included in Section 3.5. Finally, the sizedependent crystallinity of Pt nanoparticles is presented in Section 3.6. 


\subsubsection{Time-related Growth}

The duration of sputtering plays a major role in determining nanoparticle growth characteristics. Figure 2-9 (a-f) depicts the size and density of Pt nanoparticles synthesized at a $23.8^{\circ}$ target angle using different deposition times at a fixed power of $30 \mathrm{~W}$. From Figure 2-5(b), for a target angle of $23.8^{\circ}$, it can be seen that the main deposition flux is away from the substrate, and the low metal atom density region of the deposition flux is utilized for Pt nanoparticle formation minimizing thermalization of in-flight metal atoms. When the deposition time is increased, initial growth starts by cluster formation at nucleation sites followed by high energy Pt atoms arriving at the substrate and exhibiting surface diffusion and coalescence (Figure 2-9 (a-b)).

Until $10 \mathrm{~s}$ of deposition time, the coalescence process is not recognizable as the interparticle distance is large with numerous available nucleation sites. Due to a large interparticle distance, when a Pt atom arrives, it binds to a new nucleation site instead of finding another particle to coalesce. It is important to note that the actual mean diameter can possibly be smaller than the characterized value for a $5 \mathrm{~s}$ deposition time. Thus, by extrapolating the growth curve of the Pt nanoparticles with respect to deposition time ((Figure 2-9 (g) and (i)), the mean nanoparticle size was predicted to be around $0.5 \mathrm{~nm}$, whereas the calculated mean value based on observed particles from HRTEM images was $0.7 \mathrm{~nm}( \pm 0.12)$. This is an overestimation because particles smaller than $0.5 \mathrm{~nm}$ could not be accurately accounted for due to the resolution limits of the HRTEM.

To understand the time dependent growth mechanism of Pt nanoparticles, the ensemble of particles needs to be modelled using statistical analysis tools to determine 
scaling theories using rate equations. In nanoparticle growth literature, scaling theories indicate that diameter,

$$
\mathrm{d} \propto \mathrm{t}^{\mathrm{x}}
$$

where $t$ is the deposition time and $x=1 / D_{c}$, where $D_{c}$ is the dimensionality of the cluster, with $D_{c}=3$ for 3-dimensional clusters and 2 for 2-dimensional clusters. In Eq.1, the ' $\mathrm{x}$ ' term holds a value of 1/2 for two-dimensional particle growth, and 1/3 for three-dimensional particle growth [28]. In analogy with this, Beysens et al. [44] previously reported that the evolution of the pattern of droplets when water condenses on glass (breath figures) is quantitatively similar to that observed when vapors of metals, in their case silver, condense on solids. They [44] reported that the average droplet radius in the case of breath figure studies increased following the aforementioned power law at initial stages when droplets grew and nucleated without any coalescence. They further claimed that this relationship follows from scaling arguments that can be applied irrespective of the underlying growth mechanisms.

Three successive stages of growth were identified: - 1) Droplets nucleate and grow with no significant interactions between them (the nucleation stage). In this regime, the average droplet radius increases according to a power law. 2) When the surface coverage of these nucleated cluster/particles becomes sufficiently large, the droplets interact by coalescence, which makes them move on the surface and combine to form bigger particles leading to an increase in mean droplet size and reduction in droplet number density. 3) At a time when the actual distance between droplets reaches a critical value, small droplets begin to appear between the large droplets. This new generation of droplets grows in turn 
through each of the aforementioned stages of growth [44]. This application of the power law and correlation of development of breath figures to vapor phase metal nanoparticle growth has made a significant impact on understanding nucleation and growth dynamics of metal nanoparticles. 

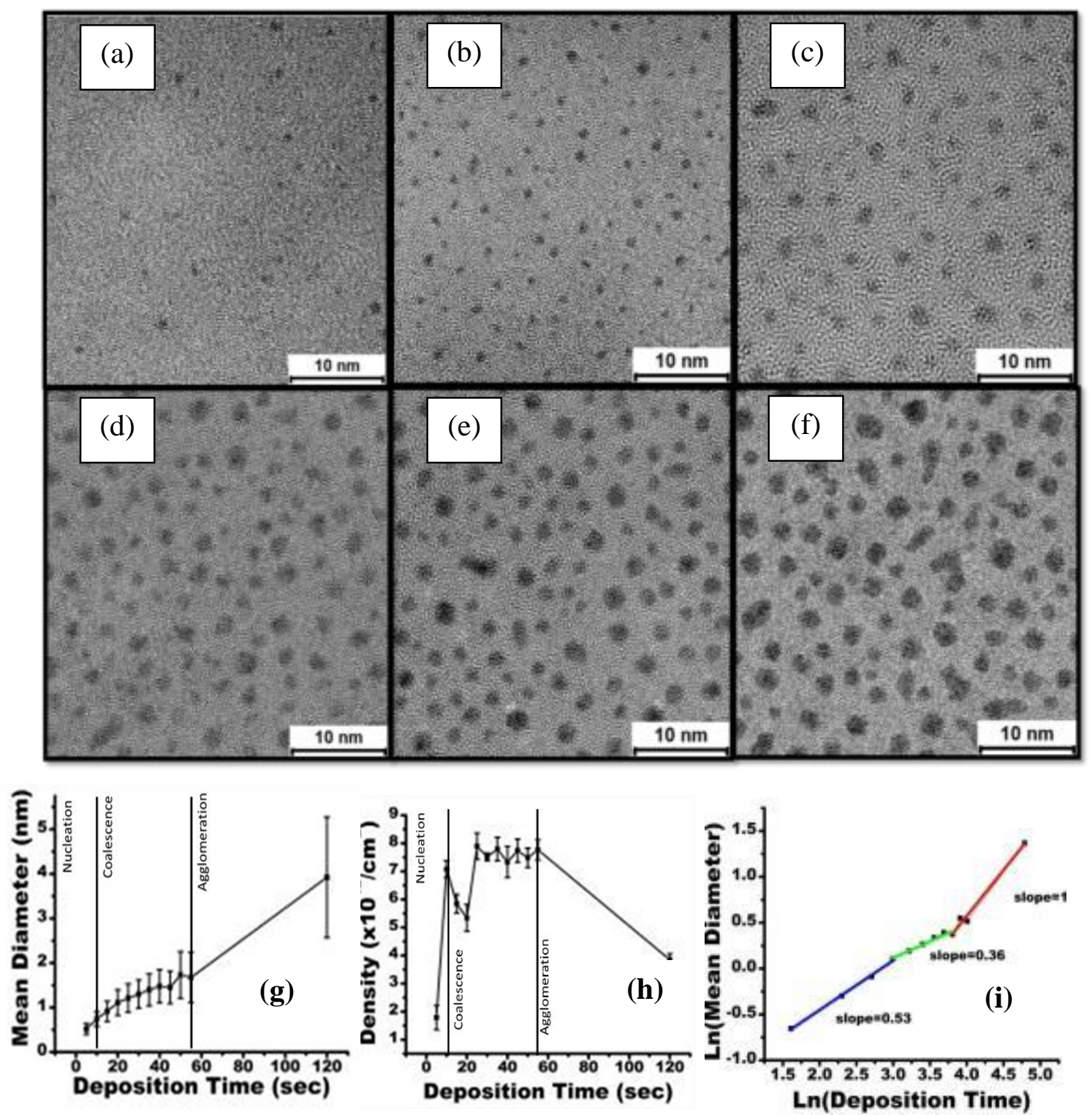

Figure 2-9: HRTEM images of Pt nanoparticles deposited at $23.8^{\circ}$ for (a) $5 \mathrm{~s}$, (b) $10 \mathrm{~s}$, (c) $20 \mathrm{~s}$, (d) 30 $\mathrm{s}$, (e) $45 \mathrm{~s}$ and (f) $55 \mathrm{~s}$. Plot of (g) mean diameter vs. deposition time and (h) mean density vs. deposition time. Linear-fit modeling (rate equation) of time-related growth mode (i). 
In order to model the time dependent nanoparticle growth characteristics in line with the observations reported by Beysens et al. [44], the power law was applied to the obtained HRTEM data. Figure 2-9(i) shows the plot of a linear model following,

$\log \mathrm{d} \propto \mathrm{x} \log \mathrm{t}$

where $\mathrm{x}$ is the slope of the fit. Different regions were modelled separately using statistical analysis on the obtained experimental data to understand the growth model. Notably, three distinct growth regimes (nucleation, coalescence and agglomeration) were observed while using the power law to model the nanoparticle characteristics obtained from HRTEM. A slope of 0.53 was observed for the $5 \mathrm{~s}-20 \mathrm{~s}$ growth regime predicting it to be a 2dimensional cluster growth. A slope of 0.35 (approximately 1/3) was observed in $25 \mathrm{~s}-45$ s regime, indicating a 3-dimensional growth model in this time regime. Beyond $45 \mathrm{~s}$, the particles started to agglomerate which changed the exponential factor to 1.17 with a sudden increase in mean diameter.

These observations are similar to the one reported by Beysens and others [28, 44]. However, our work is unique from previous works on nanoparticle growth in the following two respects: 1) achieving relatively narrow size distribution of the particles and 2) understanding of $2 \mathrm{D}$ and $3 \mathrm{D}$ growth process in terms of non-crystalline cluster (sub - 1 $\mathrm{nm}$ ) formation and subsequent crystal phase transformation. These issues are addressed in the following sections in detail.

\subsubsection{Homogenous size distribution of sputtered nanoparticles}

As discussed in the introduction, obtaining homogenous size distributions (minimal size variation) in the sub- $2 \mathrm{~nm}$ is essential for understanding of the fundamental role of 
nanoparticle size in influencing a multitude of phenomena such as quantum confinement and charge storage as well as catalytic activity. Figure 2-10 shows the particle size distribution for different deposition times at $30 \mathrm{~W}$ sputtering power. Table 2-3 shows average nanoparticle diameter and the standard deviation. The $5 \mathrm{~s}$ and $10 \mathrm{~s}$ particles showed the least deviation from the mean particle size due to the dominance of nucleation within the growth processes and lack of coalescence. The standard deviation in the particle size increased with increase in deposition time due to the coalescence and growth of nano particles eventually leading up to the agglomeration regime at later stages of growth. However, even with larger size particles, it is essential to note that the particle density was higher and the size distribution was narrower compared to previously reported results in published literature, particularly those using high-temperature dewetting processes to synthesize nanoparticles [14, 19, 21, 23, 26-28].

Table 2-3: Pt Nanoparticle mean-diameter and its standard deviation with time variation.

\begin{tabular}{ccc}
\hline $\begin{array}{c}\text { Time } \\
(\mathbf{s})\end{array}$ & $\begin{array}{c}\text { Diameter } \\
(\mathbf{n m})\end{array}$ & $\begin{array}{c}\text { Std. Dev } \\
(\mathbf{n m})\end{array}$ \\
\hline $\mathbf{5}$ & 0.52 & 0.12 \\
$\mathbf{1 0}$ & 0.74 & 0.17 \\
$\mathbf{1 5}$ & 0.91 & 0.22 \\
$\mathbf{2 0}$ & 1.11 & 0.28 \\
$\mathbf{2 5}$ & 1.21 & 0.31 \\
$\mathbf{3 0}$ & 1.30 & 0.31 \\
$\mathbf{3 5}$ & 1.39 & 0.35 \\
$\mathbf{4 0}$ & 1.47 & 0.37 \\
$\mathbf{4 5}$ & 1.45 & 0.36 \\
$\mathbf{5 0}$ & 1.73 & 0.52 \\
$\mathbf{5 5}$ & 1.67 & 0.56 \\
\hline
\end{tabular}



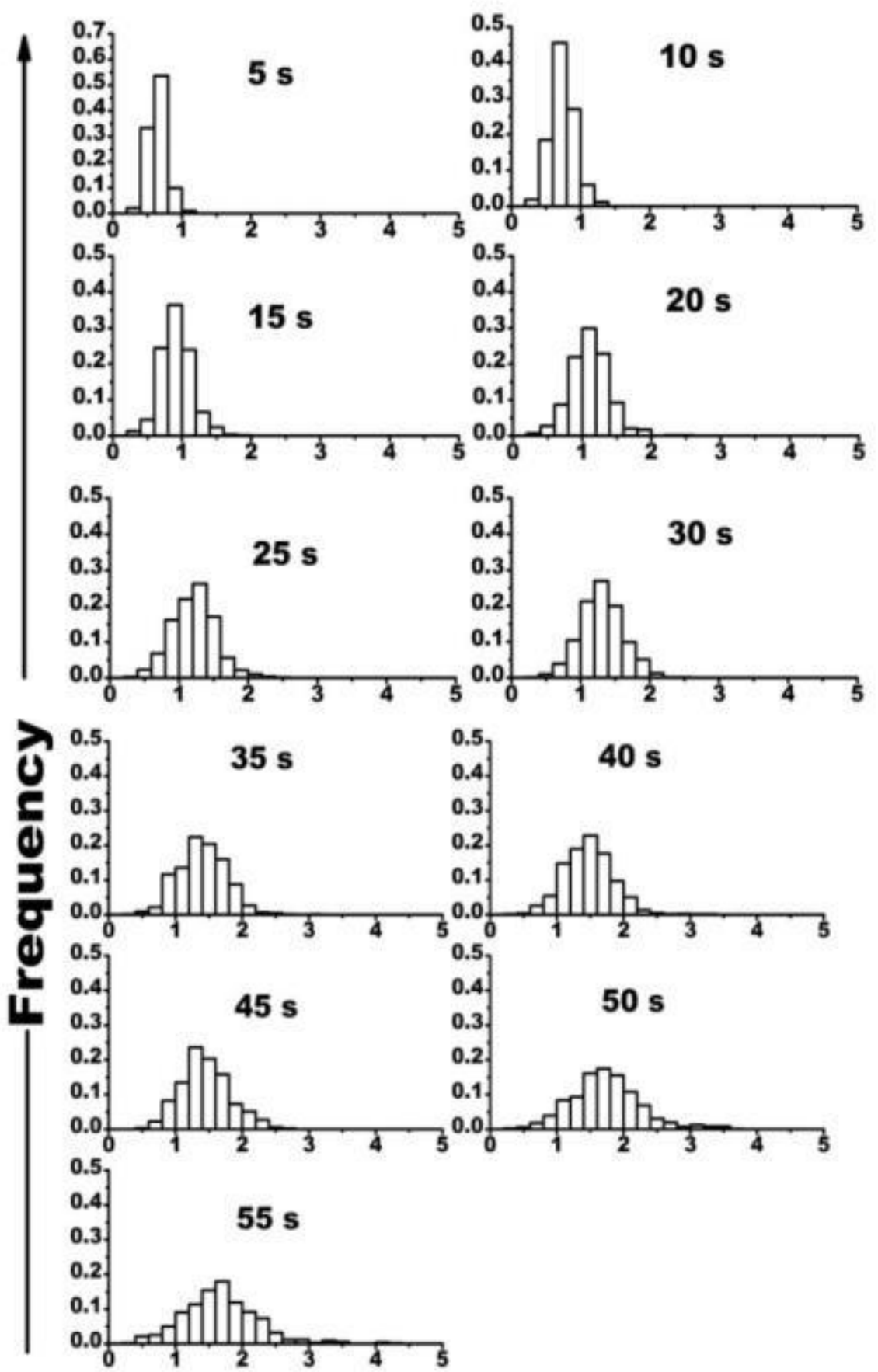

Mean Diameter

Figure 2-10: Particle size distribution for $30 \mathrm{~W}$ deposition power (target angle=23.80) and for $5 \mathrm{~s}$, 10s, $15 \mathrm{~s}, 20 \mathrm{~s}, 25 \mathrm{~s}, 30 \mathrm{~s}, 35 \mathrm{~s}, 40 \mathrm{~s}, 45 \mathrm{~s}, 50 \mathrm{~s}$ and $55 \mathrm{~s}$. 


\subsubsection{Target angle dependent nanoparticle growth}

As discussed previously in relation to sputtering time, the target angle also plays a major role in determining sputtered Pt nanoparticle characteristics. Figure 2-11 shows the resulting plot of mean diameter and particle density with different target angles (TA) deposited using the parameters listed in Figure 2-5. Changing the angle of the target predominantly controls the density and the energy of the arriving atoms/clusters on the substrate surface. As metal atoms travel from the target to the substrate, deposition flux will be relatively uniform with higher density metal atoms in the area equivalent to the target diameter and will gradually disperse out radially towards chamber walls with lower density atoms[8]. Here, for lower target angles, with dissipation of the metal atom flux outside the main focal beam (peripheral regions of the main deposition flux), the metal atom density decreases, and thus, the probability of thermalization of sputtered metal atoms reduces, increasing the energy of the arriving atoms at the substrate surface $[39,40]$.

With this change in target angle, one can constitute two different deposition regimes for sputtered metal atoms/clusters based on either low or high metal atom flux density. For a low metal atom density regime (reduced thermalization) the incident atom energy exceeds the $\mathrm{Al}_{2} \mathrm{O}_{3}$ surface binding energy resulting in surface diffusion, and for high density metal atom regime (high thermalization) the incident energy of metal atoms/clusters is lower than the surface binding energy, resulting in relatively low surface diffusion. 

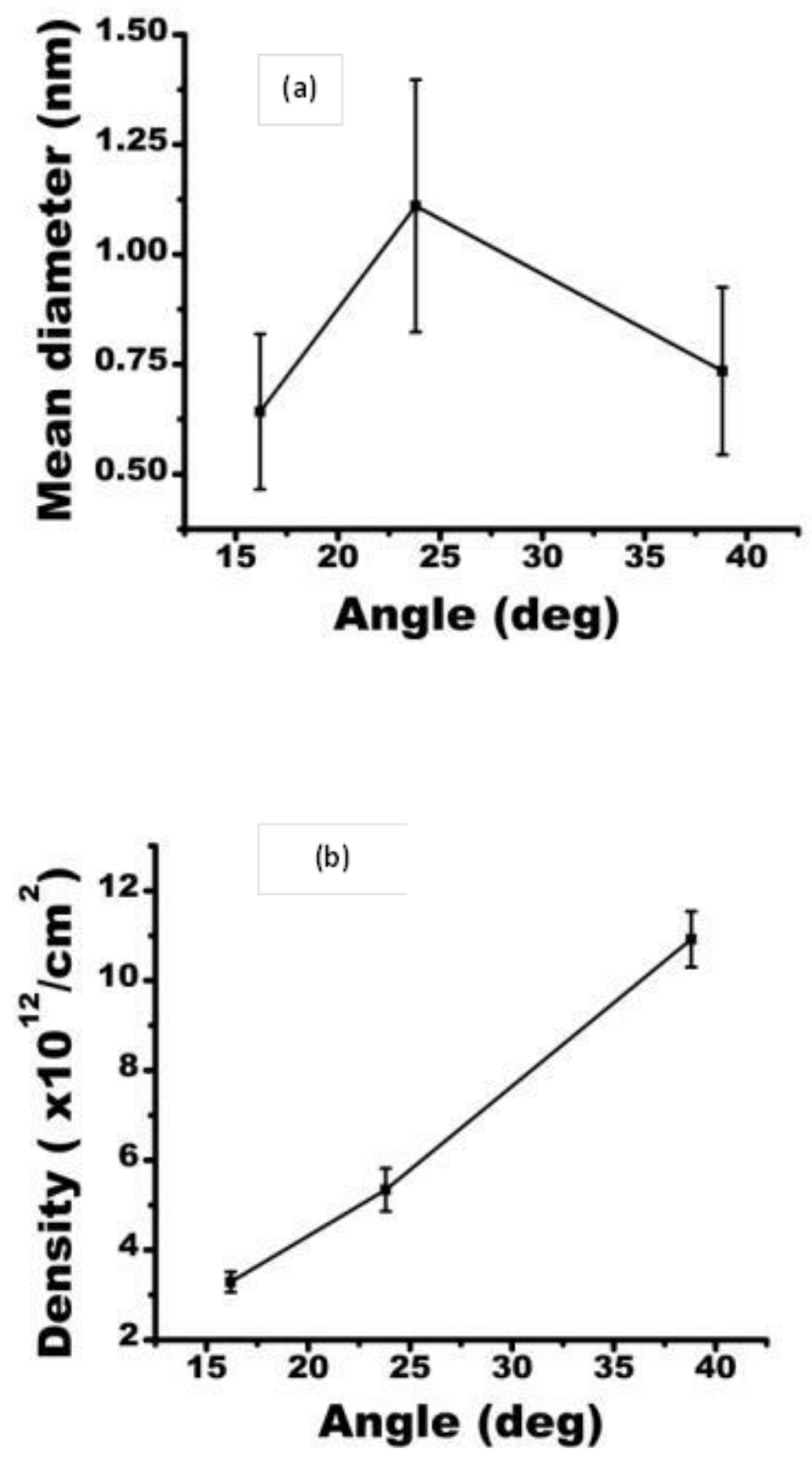

Figure 2-11: Plot showing (a) mean diameter and (b) density with different target angles 
Along with the energy of incident metal atoms/clusters, their interaction with different sites of $\mathrm{Al}_{2} \mathrm{O}_{3}$ ( $\mathrm{Al}$ or $\mathrm{O}$ atoms) may also influence the adhesion of $\mathrm{Pt}$ atoms/clusters to $\mathrm{Al}_{2} \mathrm{O}_{3}$ surface [46], and hence the nucleation and growth process. Overall, at lowest target angle

of $16.2^{0}$, the $20 \mathrm{~s}$ deposition falls in the nucleation governed growth regime with smallest diameter and lowest density of clusters due to the lower concentration of high energy atoms arriving at the substrate, as shown in Figure 2-11. For $23.8^{0}$ target angle, the 20 s deposition falls in the coalescence and growth regime with increased diameter and density of the clusters compared to $16.2^{0}$ target angle due to the high density of high energy atoms arriving at the substrate. For highest target angle of $38.8^{0}$, high density of low energy atoms are generated due to dominance of thermalization process. Compared to $23.8^{0}$ target angle, a reduced diameter and increased density of clusters is observed in $20 \mathrm{~s}$ deposition due to the nucleation and growth of clusters without coalescence (Figure 8).

\subsubsection{Time dependent growth at different target angles}

From Figure $2-12$ (a) at $16.2^{\circ}$ target angle, the metal atoms in the low deposition flux regime take part in the nanoparticle formation. Thus, the degree of thermalization of in-flight Pt atoms reduces and the sputtered Pt atoms retain their energy as they arrive at the substrate. Again, the Pt nanoparticle growth here is governed by the nucleation process followed by subsequent coalescence of Pt atoms/clusters on the surface. As the metal atom density is extremely low for this target angle, less metal atoms arrive at the substrate leading to low particle density and consequently higher- 

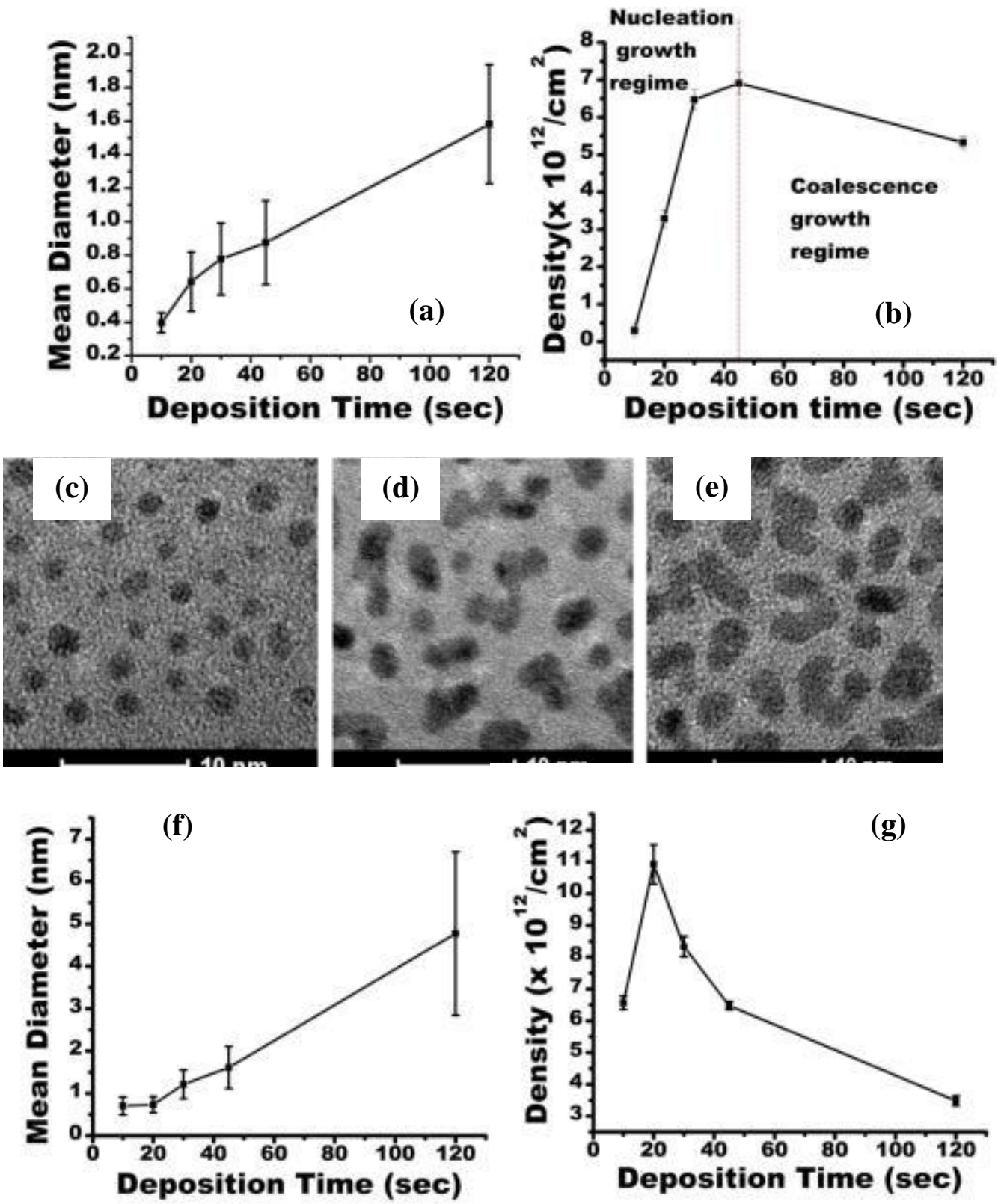

Figure 2-12: (a) mean diameter plot (TA=16.2 deg); (b) density (TA=16.2 deg); (c) 2 min at TA= 16.2 deg; (d) 2 min at TA=23.8 deg; (e) 2 min at TA=38.8deg; (f) Mean Diameter for TA=38.8 deg and (g) Density for $\mathrm{TA}=38.8 \mathrm{deg}$ 
inter-particle distance. Thus, they cannot coalesce into bigger particles through surface migration even with high energy of the arriving atom due to a higher inter-particle distance. After a deposition time of $30 \mathrm{~s}$, the inter-particle distance is such that coalescence process starts occurring as the atoms arrive at the substrate, and thus, one can observe spherical particles even at $120 \mathrm{~s}$ of deposition time with not much change in density (Figure 2-12(b)). All HRTEM images for $16.2^{\circ}$ TA for different sputtering times are provided in the Figure $2-13$

As can be seen from Figure 2-12 (c) and (e-g), the 38.8 target angle, exhibits a different growth phenomenon. At this angle, the focal area of the main deposition flux lies within the substrate location thereby exhibiting a different growth process. Due to a collimated deposition flux, the sputtered metal atoms are focused within the 2-inch diameter of the metal target. Since the metal atom density is extremely high in this focal area, the probability of thermalization drastically increases, thereby reducing the energy of the in-flight Pt atoms/clusters arriving at the substrate for nanoparticle formation. Thus, for low energy incident Pt atoms, the adatom-substrate adhesion energy dominates adatomadatom binding energy rendering surface migrations. Thus, the arriving Pt atoms nucleate on the surface without lateral diffusion. As the sputtering time is increased beyond $20 \mathrm{~s}$, the Pt nanoparticle mean size increases, and the number density drastically decreases. This can be explained by the nanoparticle growth occurring in the coalescence regime where coalescence of Pt clusters occurs as the energy released due to Pt atom - Pt cluster bond finally exceeds the Pt cluster - surface binding energy, and the Pt clusters start coalescing to from bigger particles [42]. This effect is more pronounced after $45 \mathrm{~s}$ of Pt sputtering where the Pt nanoparticle size increases and the density size drops further. As the Pt 
nanoparticle size further increases, the nanoparticle agglomerates create worm like structures and increase in overall size drastically reducing the particle density. This later stage nano-island growth is in the agglomeration regime. Figure 2-15 shows the HRTEM images for $38.8^{\circ} \mathrm{TA}$ for different sputtering times.

Another interesting nanoparticle characteristic that can be used to gain further understanding of its growth mechanism is the saturation number density. For $16.2^{\circ} \operatorname{target}$ angle, (Figure 2-12 (b)), as the deposition time is increased, it can be seen that the number density does not increase beyond $7 \times 10^{12} / \mathrm{cm}^{2}$ before saturation. This saturation of $\mathrm{Pt}$ number density is another indication of higher energy $\mathrm{Pt}$ atoms/clusters arriving at the substrate at this low target angle. For higher energy Pt atoms/cluster arriving at the surface, the average migration radius (indication of distance nanoparticles/clusters can migrate to and from their mean position on the substrate surface) for a certain-sized Pt nanoparticle/cluster is larger and thus, the inter-particle distance where coalescence is likely to occur and result in larger particles (leading to decrease in nanoparticle number density) is also larger. Therefore, the jamming limit (surface coverage of Pt nanoparticles - defined as the limit of packing in the process of random sequential adsorption in two dimensions [44]) would be smaller for lower target angles and would ultimately lead to lower saturation of nanoparticle number densities. 


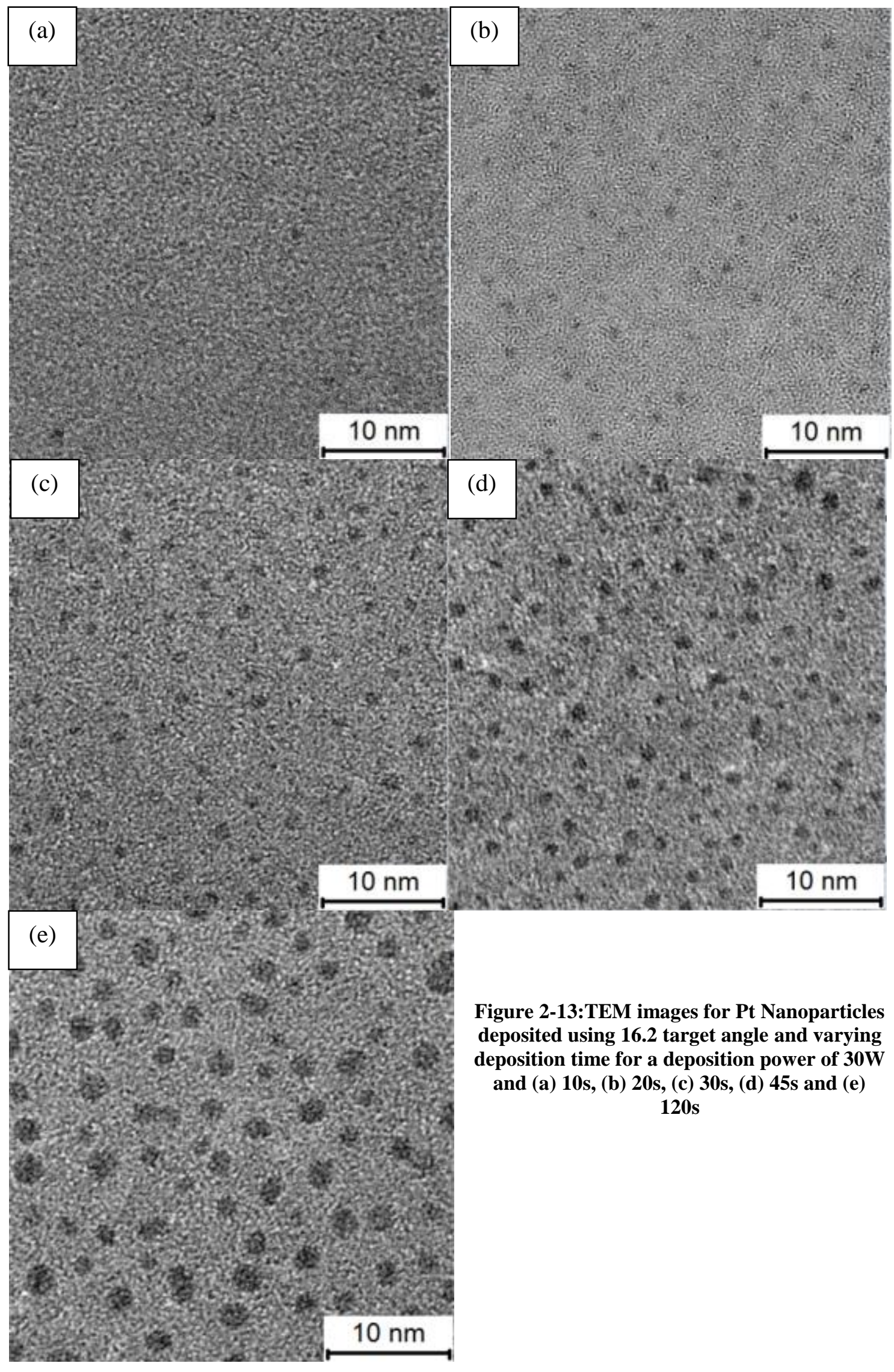



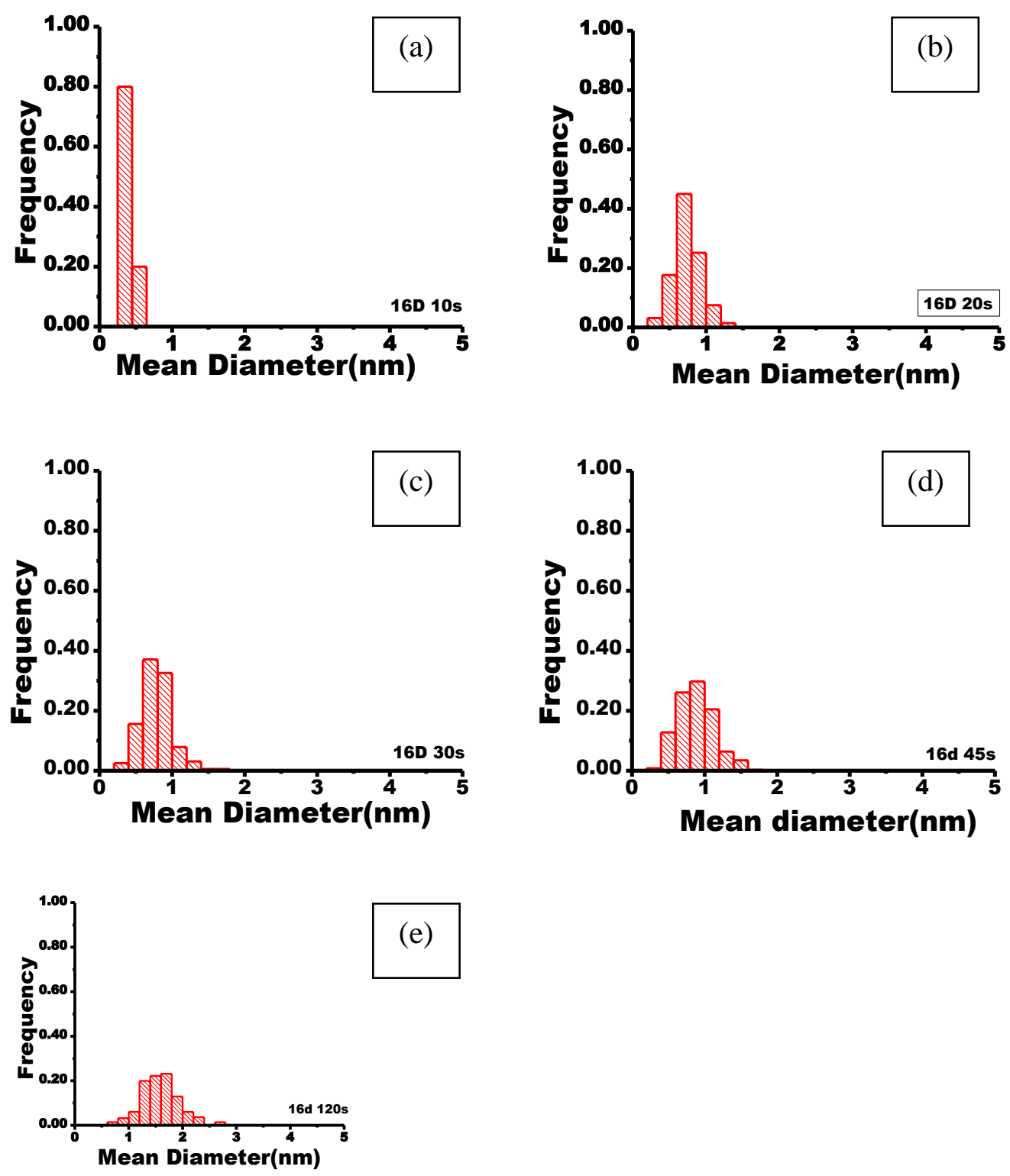

(e)

Figure 2-14: Size distribution for Pt Nanoparticles deposited using 16.2 target angle and varying deposition time for a deposition power of $30 \mathrm{~W}$ and (a) 10s, (b) 20s, (c) 30s, (d) 45s and (e) 120s. 
It is also important to note that the relative size distribution of nanoparticles grown beyond the nucleation regime would also be smaller. This is the case for $120 \mathrm{~s}$ sputtered $\mathrm{Pt}$ nanoparticles at $16.2^{\circ}$ target angle where the error bar for Pt nanoparticle size is $\sim \pm 22.15 \%$ as compared to $\sim \pm 40.25 \%$ for particles obtained after $120 \mathrm{~s}$ of sputtering at a higher angle $\left(38.8^{\circ}\right)$. This is a result of larger migration radius: Since the arriving high energy Pt atoms/clusters would have be more likely to diffuse on the surface and eventually stabilize, this would ultimately lead to narrow nanoparticle size distributions past the nucleation regime when compared to deposition conditions where the nanoparticles/clusters on the surface have lower average migration radius. For $38.8^{\circ}$ target angle, the ability to attain maximum particle number density of $1.1 \times 10^{13} / \mathrm{cm}^{2}$ with near complete surface nucleation was possible because of this characteristic behavior of low energy incident atoms. To our knowledge, this Pt nanoparticle number density is the highest number density reported in literature $[14,19,21,22,28,42]$. This higher value of number density saturation for high target angle sputtering (lower energy of incoming Pt atom/clusters due to dominant thermalization process) agrees with the explanation provided for the aforementioned contrary phenomenon observed for Pt nanoparticles sputtered at $16.2^{\circ}$ target angle. Figure 2-15,13 shows the HRTEM image and size distribution for 38.8 degree target angle. As mentioned before, this higher jamming limit (surface coverage) is a result of smaller migration radius as the arriving low energy $\mathrm{Pt}$ atoms/clusters would have a lower probability of diffusion on the surface and this would ultimately widen nanoparticle size distributions past the nucleation regime when compared to deposition conditions at lower target angles. 

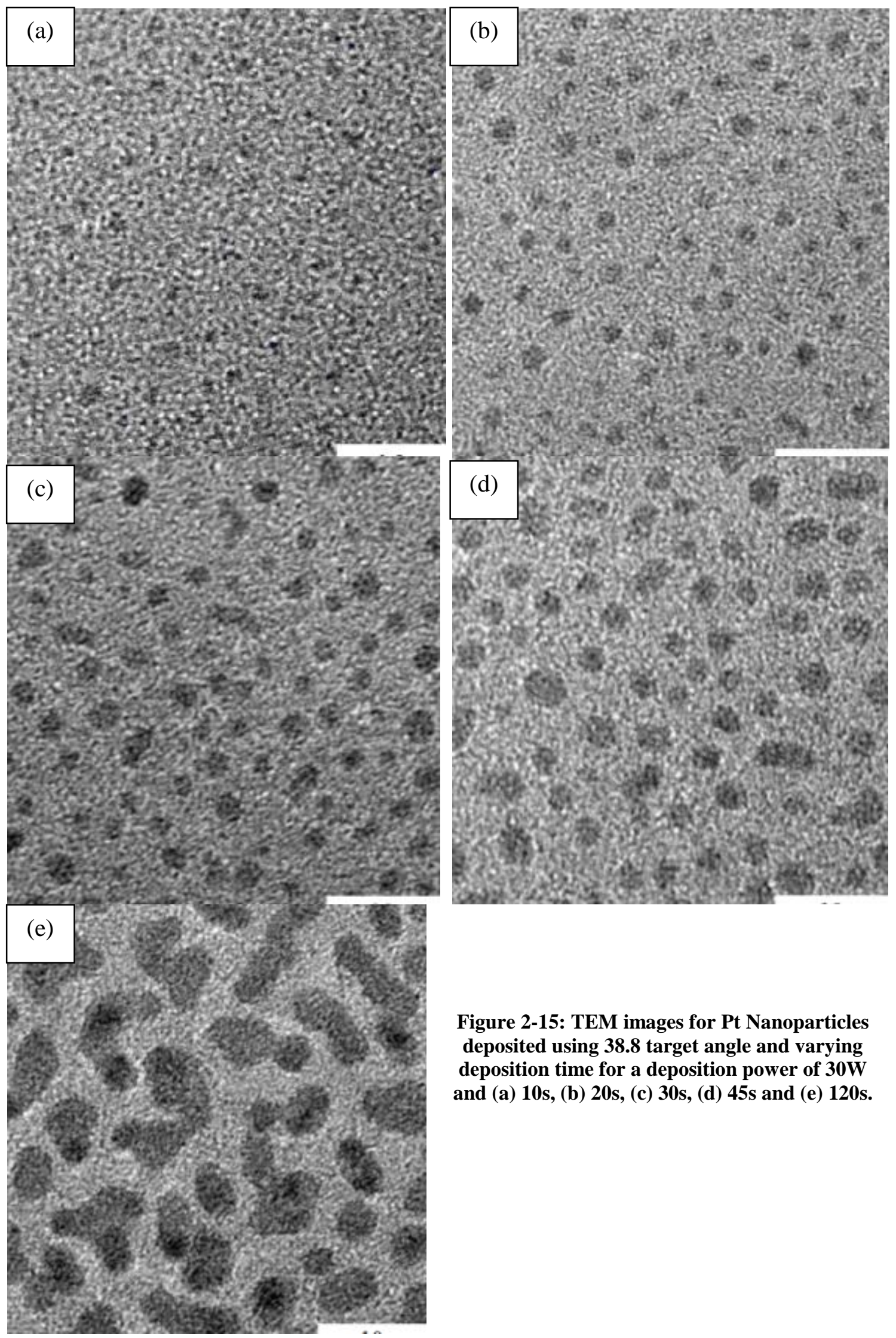

Figure 2-15: TEM images for Pt Nanoparticles deposited using 38.8 target angle and varying deposition time for a deposition power of $30 \mathrm{~W}$ and (a) 10s, (b) 20s, (c) 30s, (d) 45s and (e) 120s. 

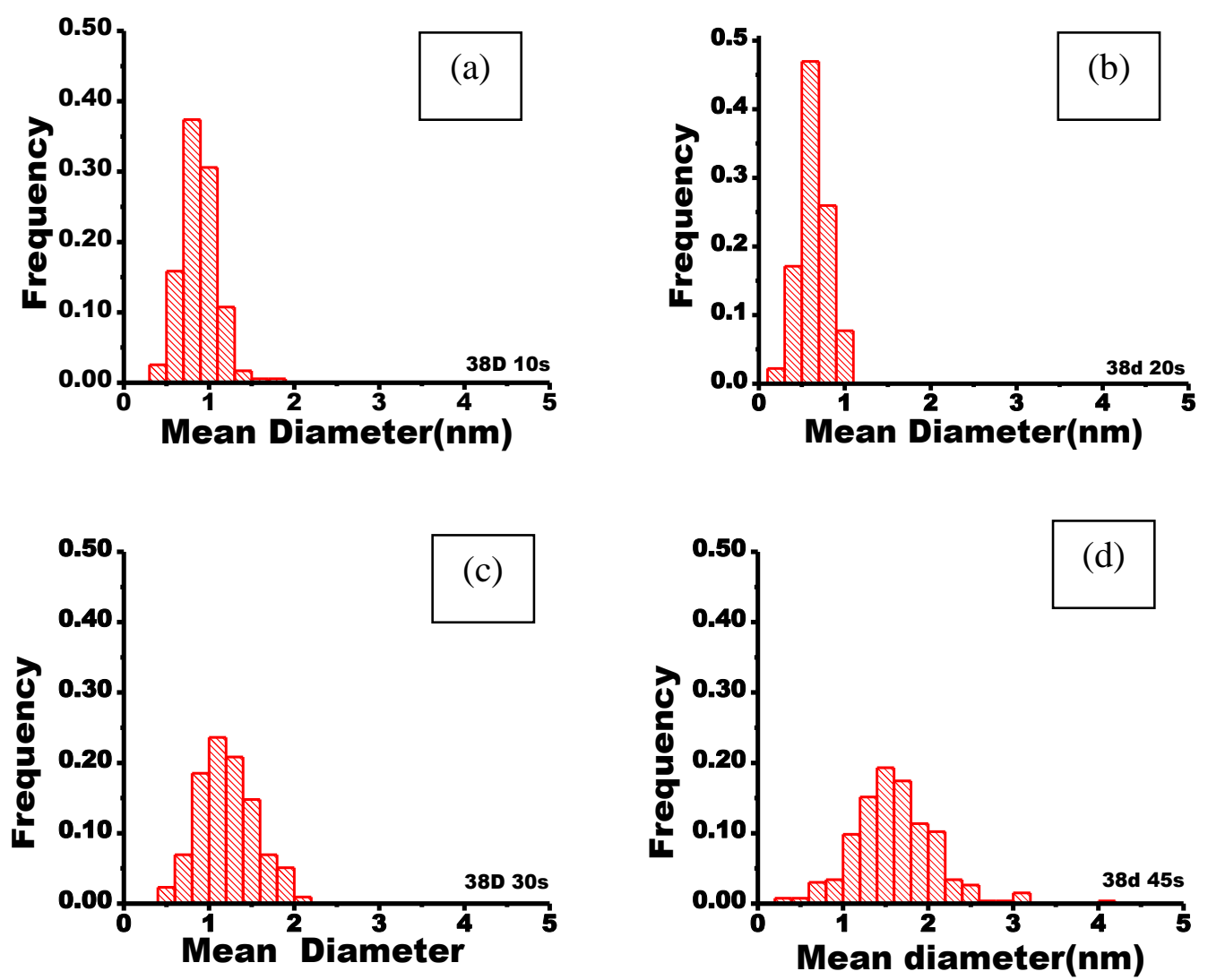

Figure 2-16:Size distribution for Pt Nanoparticles deposited using 38.8 target angle and varying deposition time for a deposition power of $30 \mathrm{~W}$ and (a) 10s, (b) 20s, (c) 30s and (d) 45s. 


\subsubsection{Substrate location variation in deposition plume}

To study the effect of sample location in the substrate holder, two locations : one in the center of the substrate holder and one in the edge was chosen to observe the variation in size and density of the Pt NP. At 16.3 deg target angle, both samples located at the center and edge of the substrate holder, are outside the main deposition flux and much farther away from the focal beam Figure 2-5. The metal atoms arriving at these locations are in the region of deposition flux with similar metal cluster size, and thus there is minimum size variation between the center and the edge. But, as it is obvious that the sample at the edge location keeps revolving (due to substrate holder rotation), which falls periodically in a higher density region of metal atoms with higher number of thermalized atoms and thus they have a higher nanoparticle density compared to the center location. It is important to note that, though the mean particle diameter was similar for both locations, there was a large deviation in the density (Figure 2-17). This potentially means non-uniform interparticle distance and thus drastically varying self-capacitance for each particle which is not desired for device applications [1]. When the target angle is increased to $23.8 \mathrm{deg}$, the focal beam of the flux lies just outside the substrate holder. Here the density of metal atoms taking part in nanoparticle formation is higher when compared to $16.3 \mathrm{deg}$. Since, the focal beam is near the substrate holder, the cluster size plays an important role at these locations. As earlier, the edge sample reaches the high density region near the focal beam periodically due to substrate rotation thereby showing higher density compared to the center sample. At this angle (23.8deg), the beam is stationed such that the flux taking part in the deposition has a minimum density variation across the substrate holder with least 
variation in size, which can be seen in Figure 2-17, where the densities of center and edge sample are closest.

Now, for the final scenario where the target angle is changed to $38.8 \mathrm{deg}$, the focal beam hits the substrate just outside the center sample and the edge sample encounters this focal beam with high density metal atoms periodically. At this stage, for the deposition time of 20 s, a high atom density flux leads to nucleation in early stages and rapid particle growth, which results in an increase in particle size and decrease in density compared to the sample at the center (Figure 2-17). On the other hand, the center sample sees a lower density flux resulting in slow nucleation and maximum density occupation. Though this gives highest density at the center location, it still can't be used due to a wide size distribution and varying density across the substrate. However, considering placing the samples only at the center, this high particle density can have various applications in optics and electrochemistry.
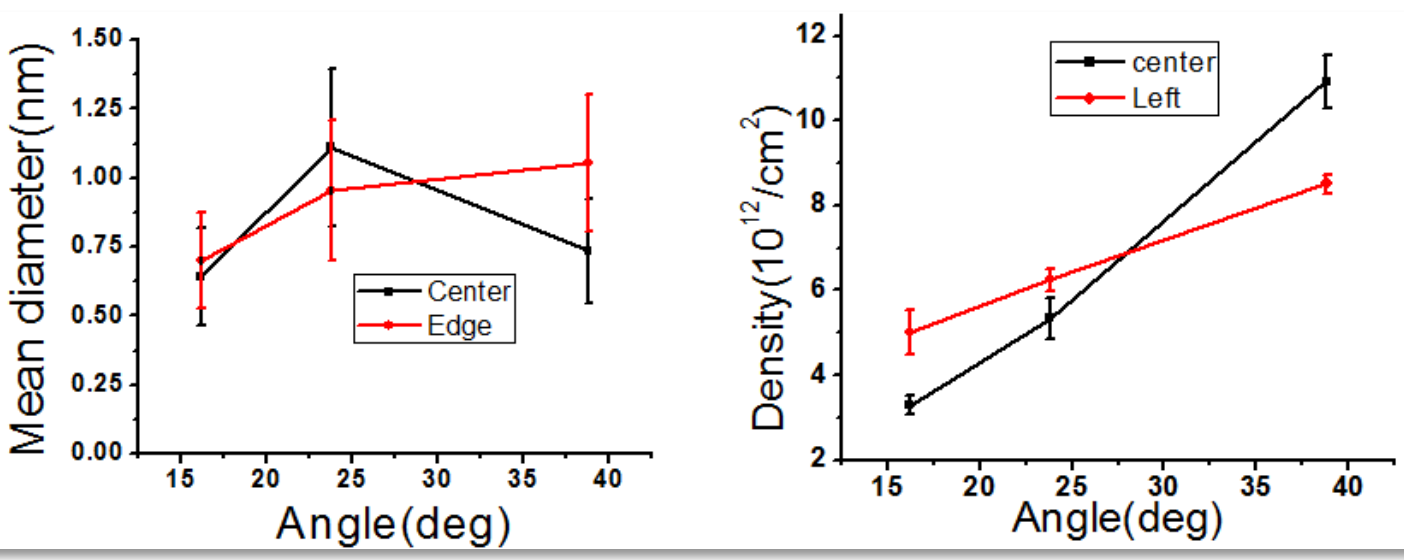

Figure 2-17: Plot for samples at different substrate location: (a) mean diameter plot and (b) Density plot. 


\subsubsection{Sputtering power dependent growth}

Along with sputtering time and target angle, the sputtering power also plays an important role in determining sputtered Pt nanoparticle characteristics. Figure 2-18 shows the size and density with respect to change in deposition power. Deposition time of $10 \mathrm{~s}$ was kept constant with power varying from $20 \mathrm{~W}$ to $60 \mathrm{~W}$ at a target angle of $23.8^{\circ}$. This deposition time was chosen to observe all particle growth regimes with increasing power.

The growth behavior of Pt nanoparticles with respect to change in power can be explained based on the aforementioned thermalization process. As change in power is directly related to the number of Ar atoms getting ionized, when the power increases, the ionization of the Ar gas increases, increasing the in-flight sputtered metal atom density in the deposition flux. Thus, with increase in power at a fixed Ar gas pressure; there will be more energetic atoms in the flux accelerating the thermalization effects within the deposition flux. As discussed previously, the energy associated with the incident metal atoms can potentially exhibit different processes for nanoparticle formation based on the incident metal atom energy. 

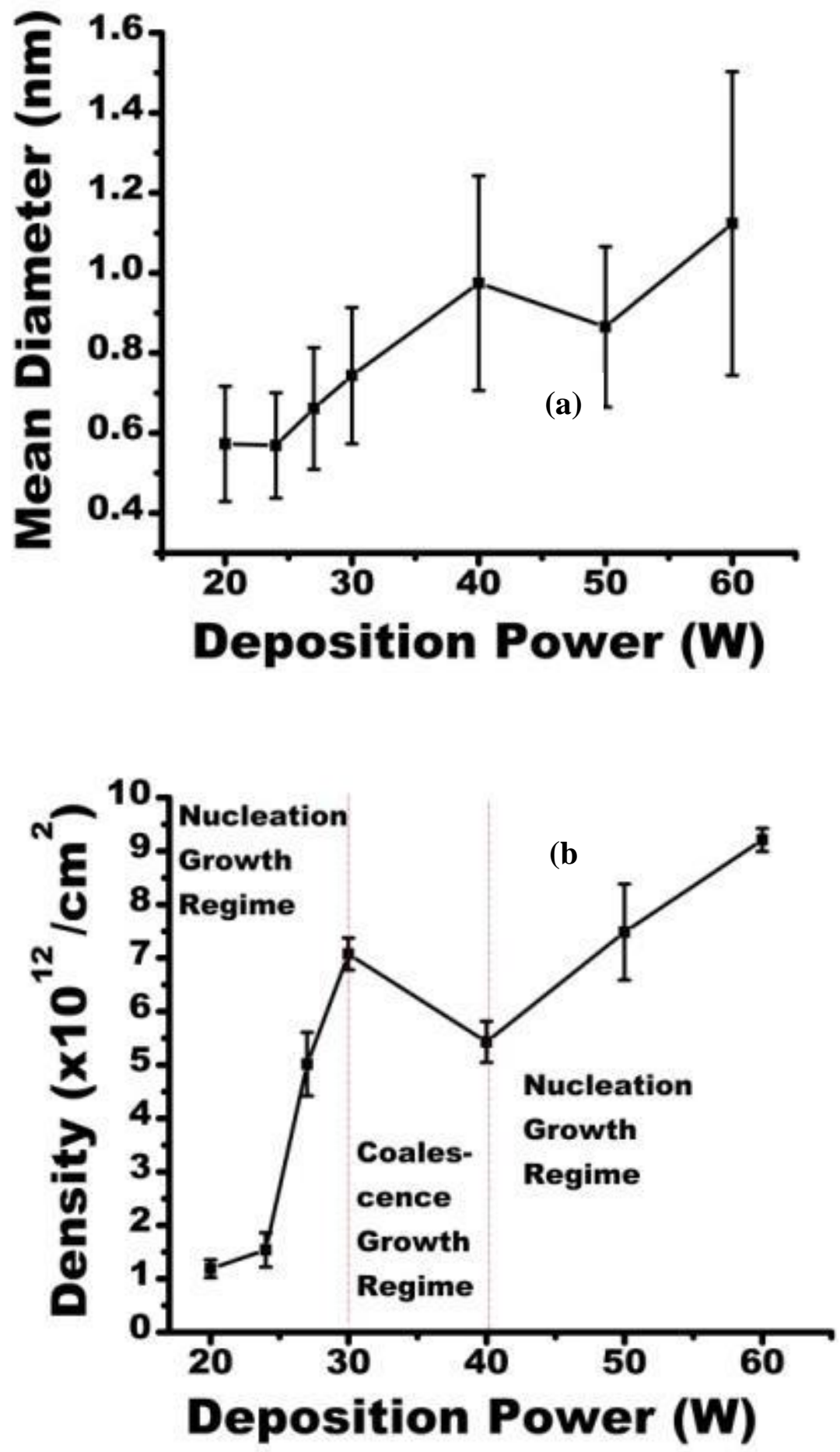

Figure 2-18: (a) Mean Diameter and (b) density plot vs. Deposition Power (at Deposition time $=10 \mathrm{~s}$ and $\mathrm{TA}=23.8 \mathrm{deg})$ 


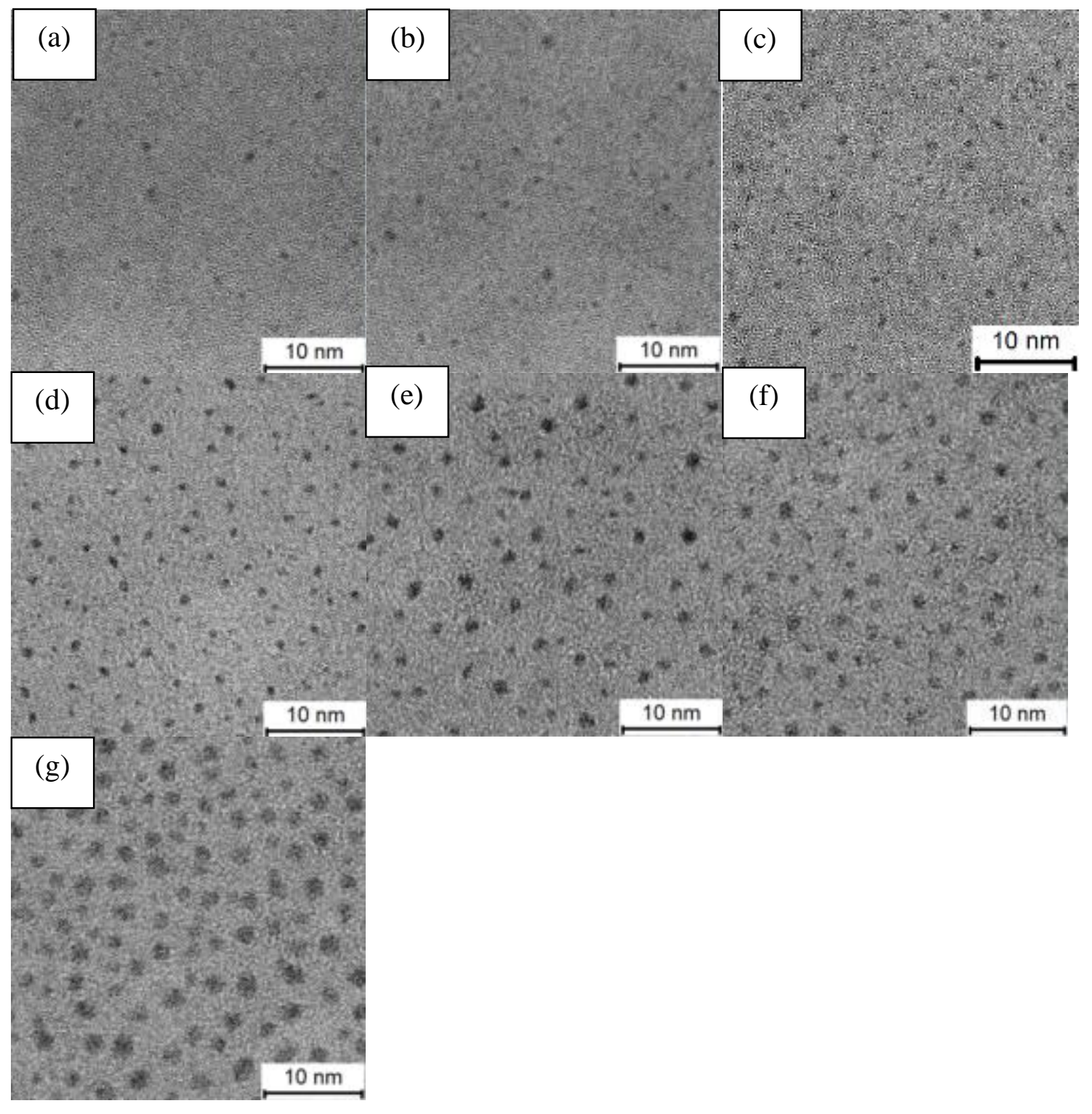

Figure 2-19:TEM images for Pt Nanoparticles deposited using varying power at deposition time of $10 \mathrm{~s}$ and target angle of $23.8^{\circ}$ for (a) $20 \mathrm{~W}$, (b) $24 \mathrm{~W}$, (c) $27 \mathrm{~W}$, (d) $30 \mathrm{~W}$, (e) $40 \mathrm{~W}$, (f) $50 \mathrm{~W}$ and (g) $60 \mathrm{~W}$. 
Table 2-4: Pt Nanoparticle mean-diameter and its standard deviation with power variation

\begin{tabular}{|c|c|c|}
\hline $\begin{array}{l}\text { Power } \\
(\mathrm{W})\end{array}$ & $\begin{array}{l}\text { Diameter } \\
(\mathbf{n m})\end{array}$ & $\begin{array}{l}\text { Std.Dev } \\
\text { (nm) }\end{array}$ \\
\hline 20 & 0.57 & 0.14 \\
\hline 24 & 0.56 & 0.13 \\
\hline 27 & 0.66 & 0.15 \\
\hline 30 & 0.74 & 0.17 \\
\hline 40 & 0.97 & 0.26 \\
\hline 50 & 0.86 & 0.20 \\
\hline 60 & 1.12 & 0.37 \\
\hline
\end{tabular}

Table 2-4 shows average nanoparticle diameter and the standard deviation at different sputtering powers. From the size distribution plots of Figure 2-13, the narrowest distribution (with standard deviation $\sim 0.13 \mathrm{~nm}$ ) is observed for low powers $(20 \mathrm{~W}-30 \mathrm{~W}$ ) as initially particles are just nucleating and growing without any coalescence process. As the power increases $(30 \mathrm{~W}-40 \mathrm{~W})$, they coalesce and the density of the particles reduces, opening new nucleation sites and thus increasing the size and size distribution.

At even higher powers, $(50 \mathrm{~W}-60 \mathrm{~W})$, the metal atom density is higher and more thermalized atoms take part in the nanoparticle formation at the substrate. In this regime, since the number of metal atoms arriving at the substrate is higher, the rate of deposition increases such that the nucleation sites fill out in a very short time and thus, the incoming low energy atom/cluster is likely to arrive atop an existing particle increasing the size 
without coalescence thereby retaining the high number density characteristic seen in $20 \mathrm{~W}$ $-30 \mathrm{~W}$ regime.

Transformation from coalescence to nucleation and growth regime just by changing the power from $40 \mathrm{~W}$ to $50 \mathrm{~W}$ is an interesting phenomenon. Since thermalization is dominant in the $50 \mathrm{~W}$ regime, due to incoming atoms just filling in nucleation sites without coalescence, the diameter is smaller and still has relatively narrower distribution at $50 \mathrm{~W}$ when compared to $40 \mathrm{~W}$ and then broadens at $60 \mathrm{~W}$ because the larger number of incoming thermalized atoms (number of $\mathrm{Pt}$ atoms sputtered is directly proportional to the sputter power) sit on new nucleation sites on the $\mathrm{Al}_{2} \mathrm{O}_{3}$ substrate or existing particle without any preference over nucleation centers. It was interesting to observe that within a window of $0.8 \mathrm{~nm}$ mean particle diameter difference, it was possible to tune the density varying from $1 \times 10^{12} / \mathrm{cm}^{2}$ to $9 \times 10^{12} / \mathrm{cm}^{2}$ simply by changing the deposition power. 

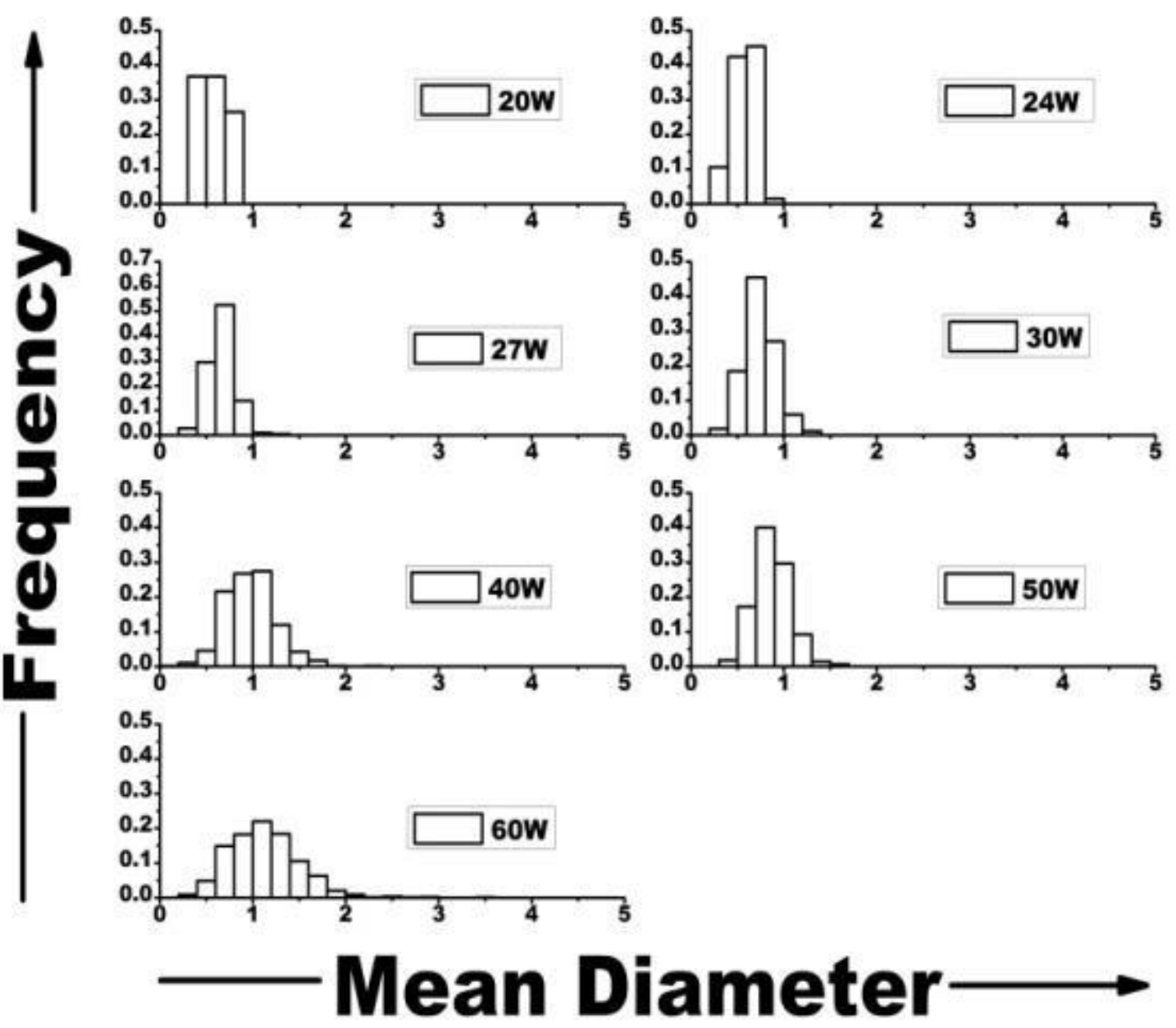

Figure 2-20: Particle size distribution for $20 \mathrm{~W}, 24 \mathrm{~W}, 27 \mathrm{~W}, 30 \mathrm{~W}, 40 \mathrm{~W}, 50 \mathrm{~W}$ and $60 \mathrm{~W}$ with a deposition time of $10 \mathrm{~s}(\mathrm{TA}=\mathbf{2 3 . 8})$ 


\subsubsection{Development of size dependent nanoparticle crystallinity}

The knowledge of crystallinity and surface faceting of metal nanoparticles is essential for application purposes - especially for catalytic applications. [47-49]. To this end, we observe evolution of size-dependent Pt nanoparticle crystalline structure with sputter times at a fixed sputtering power $(30 \mathrm{~W})$. It is important to know the size domains where these supported Pt nanoparticles make the transition from non-crystalline Pt clusters to single crystal Pt nanoparticles and later towards polycrystalline Pt nano-islands. Previously, it has been theoretically predicted that, at very small Pt nanoparticle sizes $(<1$ $\mathrm{nm})$, the surface-to-volume ratio of Pt nanoparticles in the cluster is too large to support a crystalline structure and thus the structure collapses to a more disordered structure [50]. The HRTEM, i.e., large objective--aperture bright field electron phase-contrast images of Pt nanoparticles sputtered on the Holey-carbon grids coated with $\mathrm{Al}_{2} \mathrm{O}_{3}$ films at different deposition times at $30 \mathrm{~W}$ sputtering power are shown in Figure 2-21. No sign of crystallinity was observed for resultant Pt nanoparticles formed after $10 \mathrm{~s}$ and $20 \mathrm{~s} \mathrm{of} \mathrm{Pt}$ sputtering. However, signs of nanoparticle crystallinity surfaced starting at $30 \mathrm{~s} \mathrm{Pt}$ deposition. As reported previously in [30], out of about 298 particles in the $30 \mathrm{~s}$ deposition, single $\{111\}$ or $\{200\}$ lattice fringes were identified in 29 particles, and cross-fringes in only about 5 particles. The fraction of particles with identified lattice fringes in the longer depositions was greater, i.e., between $40 \%$ and $50 \%$ for 45 s deposition. In our HRTEM image of particles on $\mathrm{Al}_{2} \mathrm{O}_{3}$ starting from $30 \mathrm{~s}$ of Pt sputtering (Figure 2-22), the spacing between the electron diffraction fringes was approximately $2.2 \AA$. 


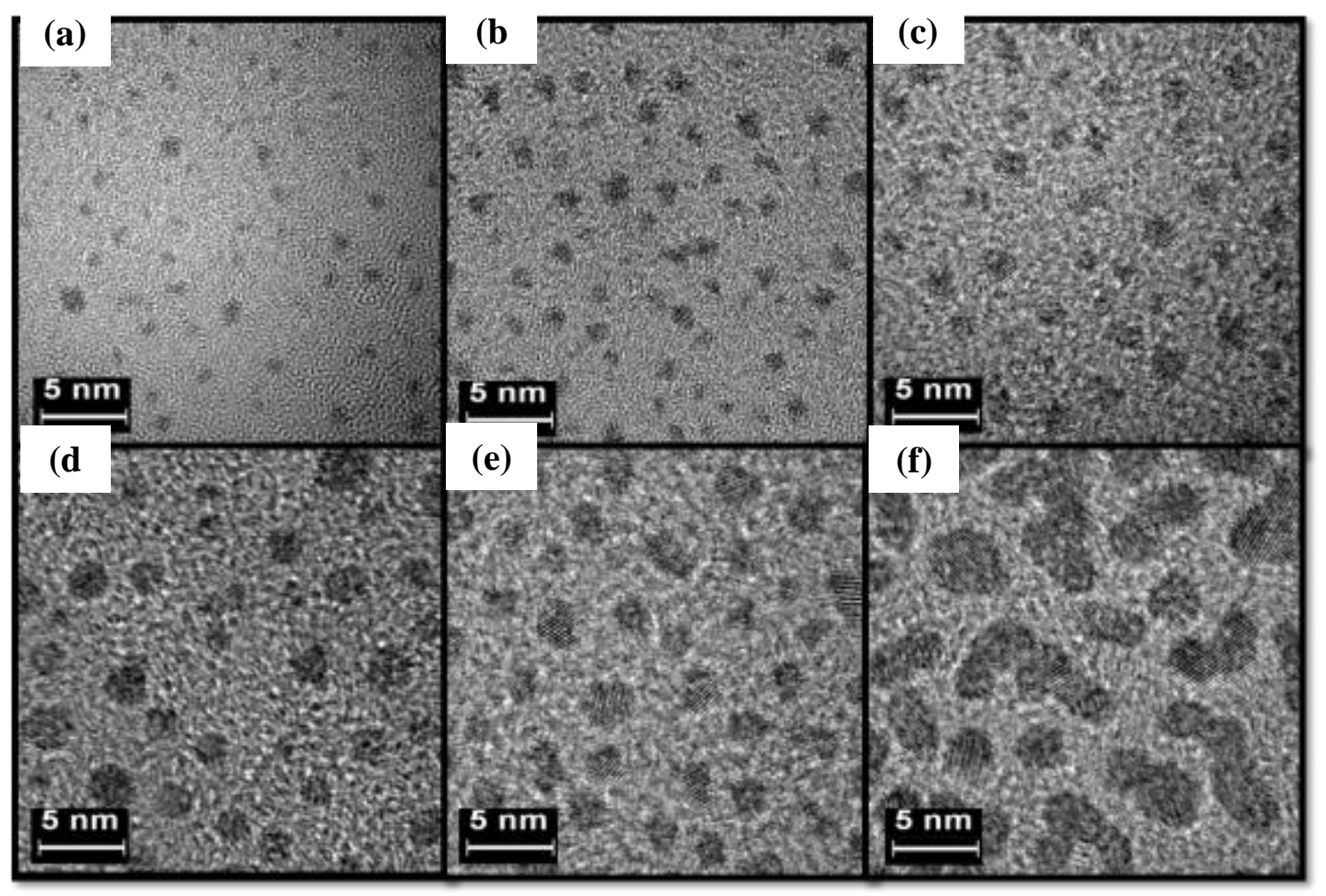

Figure 2-21:HRTEM images of Pt nanoparticles sputtered for various durations: (a) $10 \mathrm{~s}$, (b) $20 \mathrm{~s}$, (c) $30 \mathrm{~s}$, (d) $45 \mathrm{~s}$, (e) $60 \mathrm{~s}$, and (f) $120 \mathrm{~s}$ with a deposition power of $30 \mathrm{~W}$ and $\mathrm{TA}=23.8$. 
This fringe spacing corresponds to a $\{111\}$ lattice plane spacing for a FCC Pt crystal. At longer deposition times (starting at $60 \mathrm{~s}$ Pt deposition), the formation of grain boundaries arising from the agglomeration of Pt nanoparticles was observed. More detailed analysis of Pt nanoparticle size dependent crystallinity and its influence over certain catalytic processes is provided in [30]. As mentioned before, formation of crystal structure within sputtered Pt nanoparticles was observed starting at $30 \mathrm{~s}$ Pt sputtering. This sputtering time coincides with the transformation of Pt nanoparticle growth mechanism from 2-D growth to 3-D growth as discussed earlier based on the power law in Section 2.4.1. Based on the development of crystalline characteristics within these nanoparticles at different sputtering times, it can be concluded that during the nucleation phase, the Pt clusters are too small to form well-ordered lattice structures due to small size and large surface-tovolume ratio. However, the rise of single crystalline nanoparticles can be seen starting from the coalescence regime of nanoparticle growth. As the growth regime switches to the agglomeration regime, observation of grain boundaries and polycrystalline domains were observed hinting at nanoparticle growth through the process of agglomeration. Thus, it can be speculated that as the Pt clusters start forming more ordered near spherical structures, a crystalline order is established within the nanoparticle leading to the observation of crystallinity of the sputtered Pt nanoparticles. 


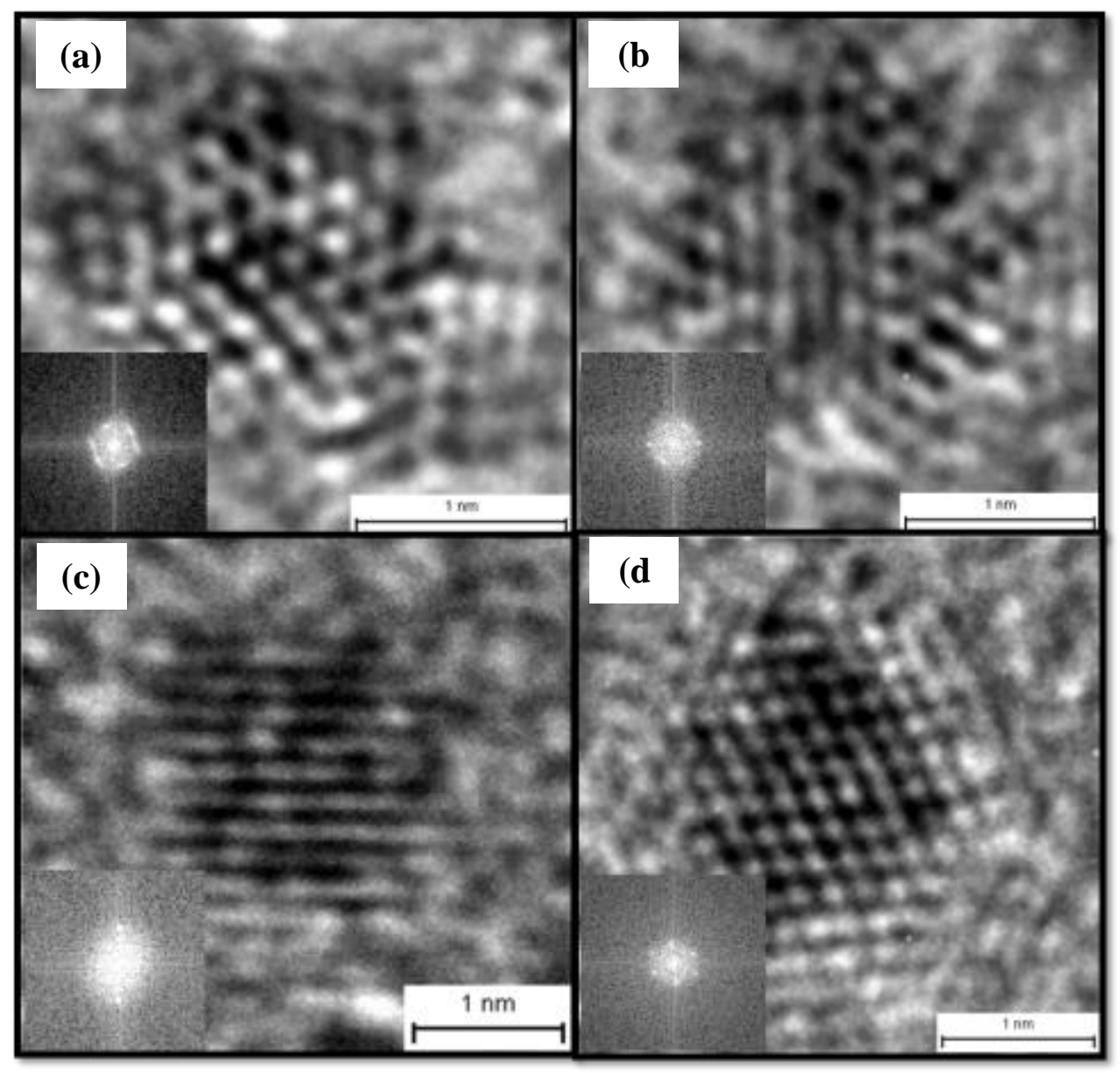

Figure 2-22: HRTEM images of Pt nanoparticles prepared by sputtering onto $\mathrm{Al}_{2} \mathrm{O}_{3}$ alongside their corresponding FFT patterns (inset) for (a, b) $30 \mathrm{~s}$ and (c, d) $45 \mathrm{~s} \mathrm{Pt}$ sputtering. The corresponding FFT patterns represent an FCC crystal along the $\langle 110\rangle$ zone axis 


\subsubsection{Longer Deposition time}

To fully understand the nanoparticle growth beyond the nanoparticle regime, longer deposition conditions were implemented to see the growth of Pt as a continuous layer of thin film. For these experiments the target angle and the deposition power were set constant at $23.8 \mathrm{deg}$ and $30 \mathrm{~W}$ respectively. The deposition time was varied to see the growth mechanism effect when extended into the agglomeration region as described in Section 2.4.1. For this study 2 min, 5 min, 10 min and 20 min deposition times were performed on $\mathrm{Al}_{2} \mathrm{O}_{3}$ coated TEM grids to analyze and characterize the growth dynamics. Figure 2-23 shows the HRTEM images for 2 minute and 5 minute deposition time. It is evident that the growth is in the agglomeration region where the Pt nanoparticles have coagulated to form $\sim 3 \mathrm{~nm}$ islands. Typically for $\mathrm{Al}_{2} \mathrm{O}_{3}$, there are nucleation sites in the substrate where particle gets bonded. With longer deposition time the particle tends to grow around these sites making bigger particles, like discussed in section 3.1.1. With 2 minute depositions, interparticle distance gets smaller and particle tends to agglomerate to form island structures. From TEM images, there is an estimation of four to five number of Pt nanoparticles agglomerated (Figure 2-23(a)). When deposition time is further increased to 5 minutes, we no longer have nanoparticles but a more radical nano-dendrimer like structures connected to each other (Figure 2-23(b)). The discreteness observed in the island for 2 minute deposition is no longer observed in the 5 minute deposition film and we start to see a transition to continuous Pt metal thin film.

For 5 minute deposition, the incoming metal atoms tend to partly stick to the Pt particles and diffuse to the surface. So, particles grow in size and a film grows around the particles leaving empty spaces or voids around the agglomerated particles. For 10 minute, 
this process continues and size of the voids decreases leading to the formation of a continuous film at 20 minute deposition. This process of attaining a continuous thin film is observed in Figure 2-23 (c-d).

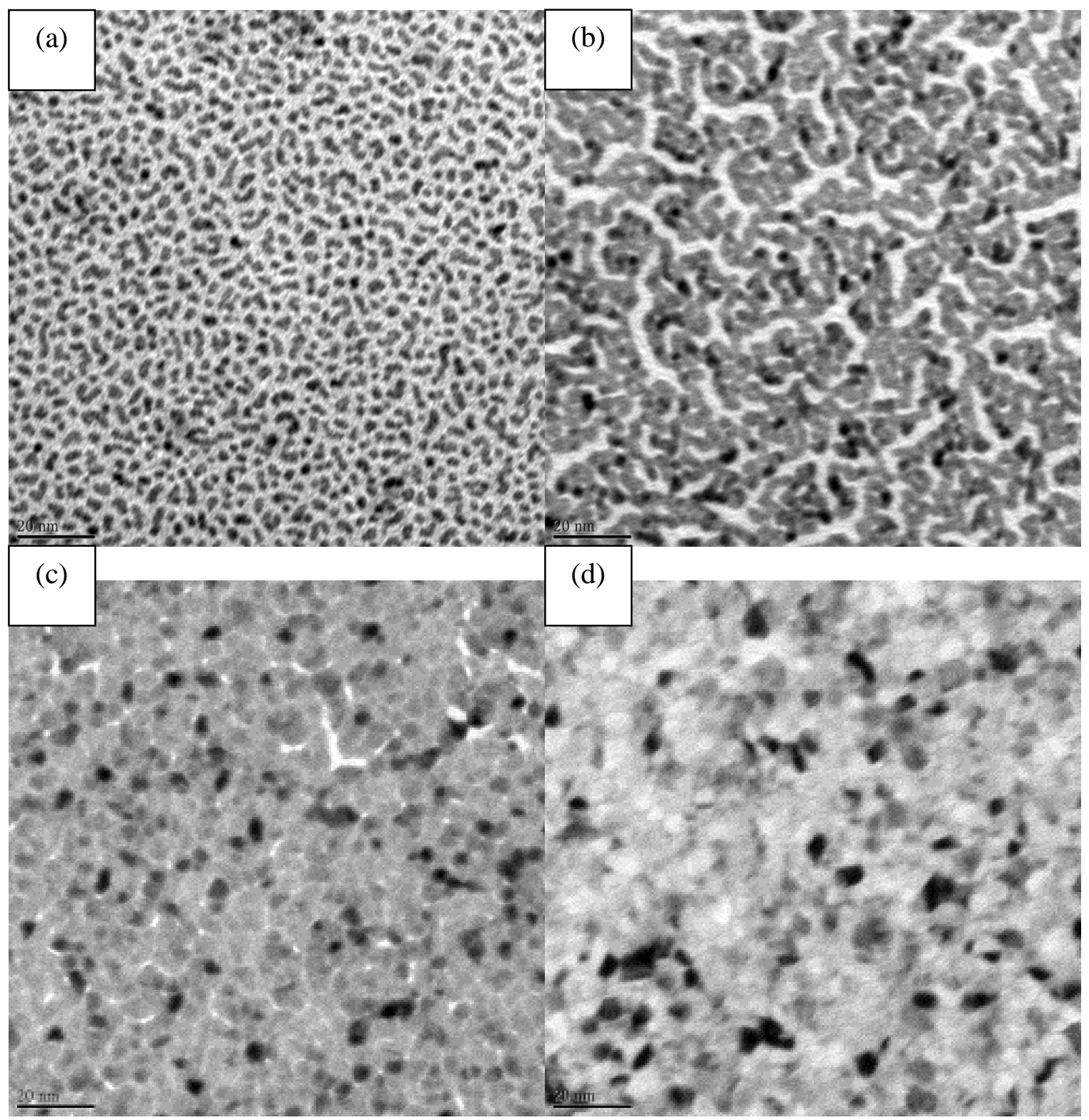

Figure 2-23:HRTEM images for (a)2 $\mathrm{min}$, (b)5 $\mathrm{min}$, (c)10 $\mathrm{min}$ and (d)20 $\mathrm{min}$ deposition using 30W deposition power and 23.8 degree target angle 


\subsection{References}

[1]. Yun, M., B. Ramalingam, and S. Gangopadhyay, Room temperature observation of size dependent single electron tunneling in a sub-2 $\mathrm{nm}$ size tunable Pt nanoparticle embedded metal-oxide-semiconductor structure. Nanotechnology, 2011. 22(46): p. 465201. [1] Y. Volokitin, J. Sinzig, L. J. de Jongh, G. Schmid, M. N. Vargaftik, and I. I. Moiseevi, "Quantum-size effects in the thermodynamic properties of metallic nanoparticles," Nature, vol. 384, pp. 621-623, 1996.

[2] S. Mandal, J. Wang, R. E. Winans, L. Jensen, and A. Sen, "Quantum Size Effects in the Optical Properties of Ligand Stabilized Aluminum Nanoclusters," The Journal of Physical Chemistry C, 2013.

[3] T. Klar, M. Perner, S. Grosse, G. von Plessen, W. Spirki, and J. Feldmann, "Nearfield optical studies of surface plasmons in single metal nanoparticles," in Quantum Electronics Conference, 1998. IQEC 98. Technical Digest. Summaries of papers presented at the International, 1998, p. 93.

[4] C. A. Foss Jr, G. L. Hornyak, J. A. Stockert, and C. R. Martin, "Templatesynthesized nanoscopic gold particles: optical spectra and the effects of particle size and shape," The Journal of Physical Chemistry, vol. 98, pp. 2963-2971, 1994.

[5] C. L. Haynes and R. P. Van Duyne, "Nanosphere lithography: A versatile nanofabrication tool for studies of size-dependent nanoparticle optics," The Journal of Physical Chemistry B, vol. 105, pp. 5599-5611, 2001. 
[6] C. J. Murphy, T. K. Sau, A. M. Gole, C. J. Orendorff, J. Gao, L. Gou, S. E. Hunyadi, and T. Li, "Anisotropic metal nanoparticles: synthesis, assembly, and optical applications," The Journal of Physical Chemistry B, vol. 109, pp. 13857-13870, 2005.

[7] S. H. Joo, S. J. Choi, I. Oh, J. Kwak, Z. Liu, O. Terasaki, and R. Ryoo, "Ordered nanoporous arrays of carbon supporting high dispersions of platinum nanoparticles," Nature, vol. 412, pp. 169-172, 2001.

[8] K. Sasaki and N. Nafarizal, "Enhancement of Ti + density in high-pressure magnetron sputtering plasmas," Journal of Physics D: Applied Physics, vol. 43, p. 124012, 2010.

[9] J. R. C. Jeff, M. Yun, B. Ramalingam, B. Lee, V. Misra, G. Triplett, and S. Gangopadhyay, "Charge storage characteristics of ultra-small Pt nanoparticle embedded GaAs based non-volatile memory," Applied Physics Letters, vol. 99, pp. 072104-3, 2011.

[10] M.Yun, B.Ramalingam, H.Zheng, and S.Gangopadhyay, "Controllable Memory Window Behavior of Size Tunable Pt Nanoparticle Embedded Organic Based Non-volatile Memory Transistors," MRS Online Proceedings Library, vol. 1359, 2011.

[11] Y. Minseong, D. W. Mueller, M. Hossain, V. Misra, and S. Gangopadhyay, "Sub2 nm Size-Tunable High-Density Pt Nanoparticle Embedded Nonvolatile Memory," Electron Device Letters, IEEE, vol. 30, pp. 1362-1364, 2009.

[12] M. Yun, B. Ramalingam, and S. Gangopadhyay, "Room temperature observation of size dependent single electron tunneling in a sub-2 nm size tunable Pt nanoparticle 
embedded metal-oxide-semiconductor structure," Nanotechnology, vol. 22, p. 465201, 2011.

[13] M. Yun, B. Ramalingam, and S. Gangopadhyay, "Multi-Layer Pt Nanoparticle Embedded High Density Non-Volatile Memory Devices," Journal of The Electrochemical Society, vol. 159, pp. H393-H399, 2012.

[14] J. Dufourcq, S. Bodnar, G. Gay, D. Lafond, P. Mur, G. Molas, J. P. Nieto, L. Vandroux, L. Jodin, F. Gustavo, and T. Baron, "High density platinum nanocrystals for non-volatile memory applications," Applied Physics Letters, vol. 92, pp. 073102-3, 2008.

[15] J. Dufourcq, P. Mur, M. J. Gordon, S. Minoret, R. Coppard, and T. Baron, "Metallic nano-crystals for flash memories," Materials Science and Engineering: C, vol. 27, pp. 1496-1499, 2007.

[16] S. Wang, X. Wang, and S. P. Jiang, "Self-assembly of mixed Pt and Au nanoparticles on PDDA-functionalized graphene as effective electrocatalysts for formic acid oxidation of fuel cells," Physical Chemistry Chemical Physics, vol. 13, pp. 6883-6891, 2011.

[17] S. Wakamatsu, J.-i. Nakada, S. Fujii, U. Akiba, and M. Fujihira, "Self-assembled nanostructure of Au nanoparticles on a self-assembled monolayer," Ultramicroscopy, vol. 105, pp. 26-31, 2005.

[18] P. Calandra, G. Calogero, A. Sinopoli, and P. G. Gucciardi, "Metal Nanoparticles and Carbon-Based Nanostructures as Advanced Materials for Cathode Application in DyeSensitized Solar Cells," International Journal of Photoenergy, vol. 2010, 2010. 
[19] M. Hirasawa, H. Shirakawa, H. Hamamura, Y. Egashira, and H. Komiyama, "Growth mechanism of nanoparticles prepared by radio frequency sputtering," Journal of Applied Physics, vol. 82, pp. 1404-1407, 1997.

[20] J. J. Brege, C. E. Hamilton, C. A. Crouse, and A. R. Barron, "Ultrasmall Copper Nanoparticles from a Hydrophobically Immobilized Surfactant Template," Nano Letters, vol. 9, pp. 2239-2242, 2009.

[21] A. R. Canário, E. A. Sanchez, Y. Bandurin, and V. A. Esaulov, "Growth of Ag nanostructures on TiO2(1 1 0)," Surface Science, vol. 547, pp. L887-L894, 2003.

[22] J. Fu and Y. Zhao, "Au nanoparticle based localized surface plasmon resonance substrates fabricated by dynamic shadowing growth," Nanotechnology, vol. 21, p. 175303, 2010.

[23] Z. Liu, C. Lee, V. Narayanan, G. Pei, and E. C. Kan, "Metal nanocrystal memories. I. Device design and fabrication," Electron Devices, IEEE Transactions on, vol. 49, pp. 1606-1613, 2002.

[24] S. P. Park, S. S. Kim, J. H. Kim, C. N. Whang, and S. Im, "Optical and luminescence characteristics of thermally evaporated pentacene films on $\mathrm{Si}$," Applied Physics Letters, vol. 80, pp. 2872-2874, 2002.

[25] Z.-Q. Tian, B. Ren, and D.-Y. Wu, "Surface-Enhanced Raman Scattering: From Noble to Transition Metals and from Rough Surfaces to Ordered Nanostructures," The Journal of Physical Chemistry B, vol. 106, pp. 9463-9483, 2002. 
[26] R. Yu, H. Song, X.-F. Zhang, and P. Yang, "Thermal Wetting of Platinum Nanocrystals on Silica Surface," The Journal of Physical Chemistry B, vol. 109, pp. 6940$6943,2005$.

[27] J. Zuo, "Deposition of Ag nanostructures on $\mathrm{TiO} 2$ thin films by RF magnetron sputtering," Applied Surface Science, vol. 256, pp. 7096-7101, 2010.

[28] P. Brault, A.-L. Thomann, and C. Andreazza-Vignolle, "Percolative growth of palladium ultrathin films deposited by plasma sputtering," Surface Science, vol. 406, pp. L597-L602, 1998.

[29] W.-N. Wang, W.-J. An, B. Ramalingam, S. Mukherjee, D. M. Niedzwiedzki, S. Gangopadhyay, and P. Biswas, "Size and Structure Matter: Enhanced CO2 Photoreduction Efficiency by Size-resolved Ultrafine Pt Nanoparticles on TiO2 Single Crystals," Journal of the American Chemical Society, 2012.

[30] S. Mukherjee, B. Ramalingam, L. Griggs, S. Hamm, G. A. Baker, P. Fraundorf, S. Sengupta, and S. Gangopadhyay, "Ultrafine sputter-deposited Pt nanoparticles for triiodide reduction in dye-sensitized solar cells: impact of nanoparticle size, crystallinity and surface coverage on catalytic activity," Nanotechnology, p. In Print, 2012.

[31] W.-J. An, W.-N. Wang, B. Ramalingam, S. Mukherjee, B. Daubayev, S. Gangopadhyay, and P. Biswas, "Enhanced Water Photolysis with Pt Metal Nanoparticles on Single Crystal TiO2 Surfaces," Langmuir, vol. 28, pp. 7528-7534, 2012/05/15 2012.

[32] Y. Minseong, R. Balavinayagam, and G. Shubhra, "Room temperature observation of size dependent single electron tunneling in a sub-2 $\mathrm{nm}$ size tunable Pt nanoparticle 
embedded metal-oxide-semiconductor structure," Nanotechnology, vol. 22, p. 465201, 2011.

[33] B. E. Roustom, G. Fóti, and C. Comninellis, "Preparation of gold nanoparticles by heat treatment of sputter deposited gold on boron-doped diamond film electrode," Electrochemistry Communications, vol. 7, pp. 398-405, 2005.

C.-L. Sun, L.-C. Chen, M.-C. Su, L.-S. Hong, O. Chyan, C.-Y. Hsu, K.-H. Chen, T.-F. Chang, and L. Chang, "Ultrafine Platinum Nanoparticles Uniformly Dispersed on Arrayed CNx Nanotubes with High Electrochemical Activity," Chemistry of Materials, vol. 17, pp. 3749-3753, 2005/07/01 2005.

D. Tonini, C. Greggio, G. Keppel, F. Laviano, M. Musiani, G. Torzo, and V. Palmieri, "Morphology of Niobium Films Sputtered at Different Target-Substrate Angle," in Proceeding of 11th Workshop on RF Superconductivity, Lubek (Germany), 2003.

[36] C. Buzea, G. Beydaghyan, C. Elliott, and K. Robbie, "Control of power law scaling in the growth of silicon nanocolumn pseudo-regular arrays deposited by glancing angle deposition," Nanotechnology, vol. 16, p. 1986, 2005.

[37] K. Robbie and M. Brett, "Sculptured thin films and glancing angle deposition: Growth mechanics and applications," Journal of Vacuum Science \& Technology A: Vacuum, Surfaces, and Films, vol. 15, pp. 1460-1465, 1997.

[38] K. V. Aeken and et al., "The metal flux from a rotating cylindrical magnetron: a Monte Carlo simulation," Journal of Physics D: Applied Physics, vol. 41, p. 205307, 2008. 
[39] K. Meyer, I. K. Schuller, and C. M. Falco, "Thermalization of sputtered atoms," Journal of Applied Physics, vol. 52, pp. 5803-5805, 1981.

[40] R. E. Somekh, "The thermalization of energetic atoms during the sputtering process," Journal of Vacuum Science \& Technology A: Vacuum, Surfaces, and Films, vol. 2, pp. 1285-1291, 1984.

[41] J. A. Venables, G. D. T. Spiller, and M. Hanbucken, "Nucleation and growth of thin films," Reports on Progress in Physics, vol. 47, p. 399, 1984.

[42] P. Andreazza, C. Andreazza-Vignolle, J. P. Rozenbaum, A. L. Thomann, and P. Brault, "Nucleation and initial growth of platinum islands by plasma sputter deposition," Surface and Coatings Technology, vol. 151-152, pp. 122-127, 2002.

[43] R. d. C. Team, "R: A language and environment for statistical computing," ed. Vienna, Austria: R foundation for statistical computing, 2013.

[44] D. Beysens, C. M. Knobler, and H. Schaffar, "Scaling in the growth of aggregates on a surface," Physical Review B, vol. 41, p. 9814, 1990.

[45] J. A. Venables, "Atomic processes in crystal growth," Surface Science, vol. 299300, pp. 798-817, 1994.

[46] C. Zhou, J. Wu, T. J. D. Kumar, N. Balakrishnan, R. C. Forrey, and H. Cheng, "Growth Pathway of Pt Clusters on $\alpha$-Al2O3(0001) Surface," The Journal of Physical Chemistry C, vol. 111, pp. 13786-13793, 2007/09/01 2007.

[47] K. Zhou and Y. Li, "Catalysis Based on Nanocrystals with Well-Defined Facets," Angewandte Chemie International Edition, vol. 51, pp. 602-613, 2012. 
[48] Z. Quan, Y. Wang, and J. Fang, "High-Index Faceted Noble Metal Nanocrystals," Accounts of Chemical Research, 2012.

[49] A. Rabis, P. Rodriguez, and T. J. Schmidt, "Electrocatalysis for Polymer Electrolyte Fuel Cells: Recent Achievements and Future Challenges," ACS Catalysis, vol. 2, pp. 864890, 2012/05/04 2012.

[50] Y. Sun, L. Zhuang, J. Lu, X. Hong, and P. Liu, "Collapse in Crystalline Structure and Decline in Catalytic Activity of Pt Nanoparticles on Reducing Particle Size to $1 \mathrm{~nm}$," Journal of the American Chemical Society, vol. 129, pp. 15465-15467, 2007.

[51] A. Dato, V. Radmilovic, Z. Lee, J. Phillips, and M. Frenklach, "Substrate-Free GasPhase Synthesis of Graphene Sheets," Nano Letters, vol. 8, pp. 2012-2016, 2008/07/01 2008.

[52] E. Yoo, T. Okata, T. Akita, M. Kohyama, J. Nakamura, and I. Honma, "Enhanced Electrocatalytic Activity of Pt Subnanoclusters on Graphene Nanosheet Surface," Nano Letters, vol. 9, pp. 2255-2259, 2009/06/10 2009.

[53] P. A. Pandey, G. R. Bell, J. P. Rourke, A. M. Sanchez, M. D. Elkin, B. J. Hickey, and N. R. Wilson, "Physical Vapor Deposition of Metal Nanoparticles on Chemically Modified Graphene: Observations on Metal-Graphene Interactions," Small, vol. 7, pp. 3202-3210, 2011.

[54] R. F. Egerton, P. Li, and M. Malac, "Radiation damage in the TEM and SEM," Micron, vol. 35, pp. 399-409, 8// 2004. 


\section{CHAPTER 3: Layer By Layer Charging In Multilevel Pt Nanoparticle Embedded Non Volatile Memory MOSCAP}

\subsection{Introduction}

Non-Volatile Memory (NVM) devices were first introduced in 1960's followed by extensive commercialization. These devices were based on a so-called floating gate design. Even at present state of the NVM roadmap, the stacked floating gate device continues to be the industry standard NVM implementation used for both standalone and embedded memories. Typically, for a solid state Floating gate NVM device, information is stored in the form of charge retention in a floating gate. Changing the number of charges in the floating gate affects the threshold voltage of the device. Consequently a change in charge can be determined by measuring this threshold voltage. Basic operation of a NVM involves either increasing or decreasing the stored charges in the floating gate, which is commonly referred to as writing and erasing operations. The commonly used phenomena to modify this associated charge in the floating gate is through either Direct tunneling or Fowler Nordheim Tunneling (FN).

Technological developments for non-volatile flash memory fabrication have focused on device area reduction and scaling down the tunneling and capping oxide thicknesses [1]. These desired design goals have imposed stringent constraints on the fabrication materials and methods with respect to current industrial-scale practices. Most commercially available flash memory devices employ Polycrystalline Silicon (pSi) or Silicon Nitride ( $\mathrm{SiN})$ as floating gate materials, which are infeasible for use as ultra-thin 
tunneling oxides due to the presence of shallow traps in the Silicon-Oxide-Nitride-OxideSilicon (SONOS) structure [2-4]. Poor charge retention properties in stacked nitride layers further cuts the number of stored electrons at each node in half, which results ultimately in a thickness scaling limitation in designing viable devices [1]. Moreover, storing charge on a single node (eg. $\mathrm{SiN}$ ) makes the memory structure prone to failure. One weak spot in the tunnel oxide is enough to create a fatal charge leakage path compromising long term reliability and retention properties of the memory architecture. The solution to the problem is to use distributed charge storage in a way that the scaling limitations are alleviated. Since the ITRS (International technology roadmap for semiconductors) forecasts charge trapping as the solution to continue NAND flash scaling, it has become essential to identify advanced materials capable of enhancing retention properties, ideally at the nanoscale $[5$, 6]. Nanocrystal related memories are one particular aspect related to the solution of this problem.

Unlike stacked Floating gate Poly-Si memory where charges are stored in a continuous layer, the nanoparticle based memory system uses layer/layers of discrete nanoparticles (made of metal or semiconductor) to store charges. This facilitates the device structure to use thinner tunneling oxide without sacrificing the endurance and retention characteristics. This is an important advantage as thinner tunneling oxides will lead to lowering operating voltages and increasing operating speeds.

Research has shown that discrete metal nanoparticles (MNPs) offer improved charge retention characteristics with respect to continuous charge trap layers due to the coulomb blockade effect and their ability to isolate the nodes electrically from their neighbors [6-12]. Additionally, metal nanoparticles are preferred over semiconductor 
nanoparticles for scaling down operational non-volatile memory devices[13, 14]. They have larger work functions, higher density of states than a semiconductor, and tunable barrier height due to various available metals[10, 13]. For semiconductor nanoparticles, at sub- $10 \mathrm{~nm}$ the bandgap increases compared to the bulk due to carrier confinement. Due to this the overall depth of the potential well of nanoparticles reduces and causes poor retention[15]. And as current trends require Effective oxide thickness(EOT) to be scaled for device miniaturization, metal nanoparticles are being embedded with high-k dielectrics for non-volatile memory applications[16]. These devices potentially exhibit smaller operating voltages, faster program/erase operations, better data endurance and long retention characteristics[16]. Specially, sub-2nm nanoparticles has shown benefit of Coulomb blockade and quantum confinement which are essential for improving charge retention characteristics for NVM devices.[17-22]

Various kinds of memory devices have been produced using embedded MNPs to improve device characteristics, especially multiple floating gates for enhanced charge storage $[10,12,21,23]$. Although an increase in memory window is observed in these devices with multiple layers, none report distinct charging steps in the large memory windows [24]. These steps will occur only after the preceding MNP layer achieves complete charge saturation for a range of bias. Upon surpassing a further threshold bias, determined by the MNP Coulomb charging energy and tunneling layer thickness, the second layer starts charging, thereby increasing the memory window. This process results in a characteristic step-like response in the flat-band voltage $\left(\mathrm{V}_{\mathrm{FB}}\right)$ vs. gate voltage plots, facilitating the identification of layer-by-layer charging and feasibility of multi-level memory architectures. 
The Coulomb charging energy of a MNP depends on the total capacitance coupled to the MNP, which is dependent on particle size and inter-particle distance between neighboring MNPs [25, 26]. Precise control of the charging energy of discrete islands depends on optimization of these properties for a layer of MNPs sandwiched between dielectric layers. In this chapter, we apply the particle deposition technique described in chapter 3 to produce a finely tuned multi-layer memory device and demonstrate controlled sequential layer charging, evidenced by step-like behavior in the $V_{\text {FB }}$ plots, through proper engineering of MNP Coulomb charging energy. The simplicity of the direct sputter deposition technique and its compatibility to standard CMOS processes makes the approach highly attractive for use in non-volatile memory devices.

\subsubsection{Basic Programming Mechanisms}

In charge-trapping memories, the charge needed to program the device has to be injected into the floating gate, i.e the nanoparticle layer. In order to change the charge or data content of NVM's, two major mechanisms have been shown to be viable: Fowler Nordheim tunneling (F-N) through thin oxides $(<12 \mathrm{~nm})$ [27] and channel hot-electron injection (CHE) [28]. Since our main devices are associated with tunneling oxides less than $12 \mathrm{~nm}$ we will only describe FN-tunneling (Figure 3-1).For nanoparticle based floating gate devices, under thermal equilibrium conditions, the floating gate is isolated from both the control gate and the substrate by the dielectrics. However, when a large bias is applied to the control gate, the two insulating layers will undergo band bending, where the thinner tunneling oxide will undergo a severe band bending and charges in the substrate will tunnel through the very thin tunneling oxide and be trapped by the floating gate, which in this case is the discrete layer of nanoparticles. As the barrier width decreases, the probability of a 
particle penetrating it rises exponentially. In sufficiently thin oxides (below $15 \AA$ ), direct quantum mechanical tunneling through the barrier can occur. Also, under oxide band bending, electrons can more readily penetrate a barrier giving rise to F-N tunneling or field emission. F-N tunneling can occur even in thicker oxides under sufficient band bending. The bending of the energy bands of the tunneling oxide and the gate oxide are different due to the thickness difference and thus the tunneling only occurs from substrate to PtNP through the tunneling oxide. Because the oxide layer above the floating gate is thicker (such that tunneling is not prominent), the charges remain trapped at the floating gate inducing shifts in flat band voltage.

\subsubsection{Basic Erasing Mechanisms}

In order to reprogram an NVM, it first has to be erased. The electrons that are injected into the floating gate are trapped by the high gate to oxide energy barrier of $3.2 \mathrm{eV}$. Since the potential-energy barrier at the oxide-silicon interface is greater than $3.0 \mathrm{eV}$, the rate of spontaneous emission of electrons from the oxide over this barrier is negligibly small. The net negative charge which remains on the floating gate shifts the $\mathrm{V}_{\mathrm{T}}$ to a positive value. Thus, again, FN tunneling is used to erase an NVM. One of the methods for erasing a floating gate, is by applying a large negative voltage at the control gate. The energy band structure will be influenced as shown in Figure 3-1(b). The applied gate voltage creates the electric field which results in a potential barrier. This barrier provides a path for the electrons to tunnel from the floating gate to the substrate through the thin gate oxide (Figure 3-1(b)). 

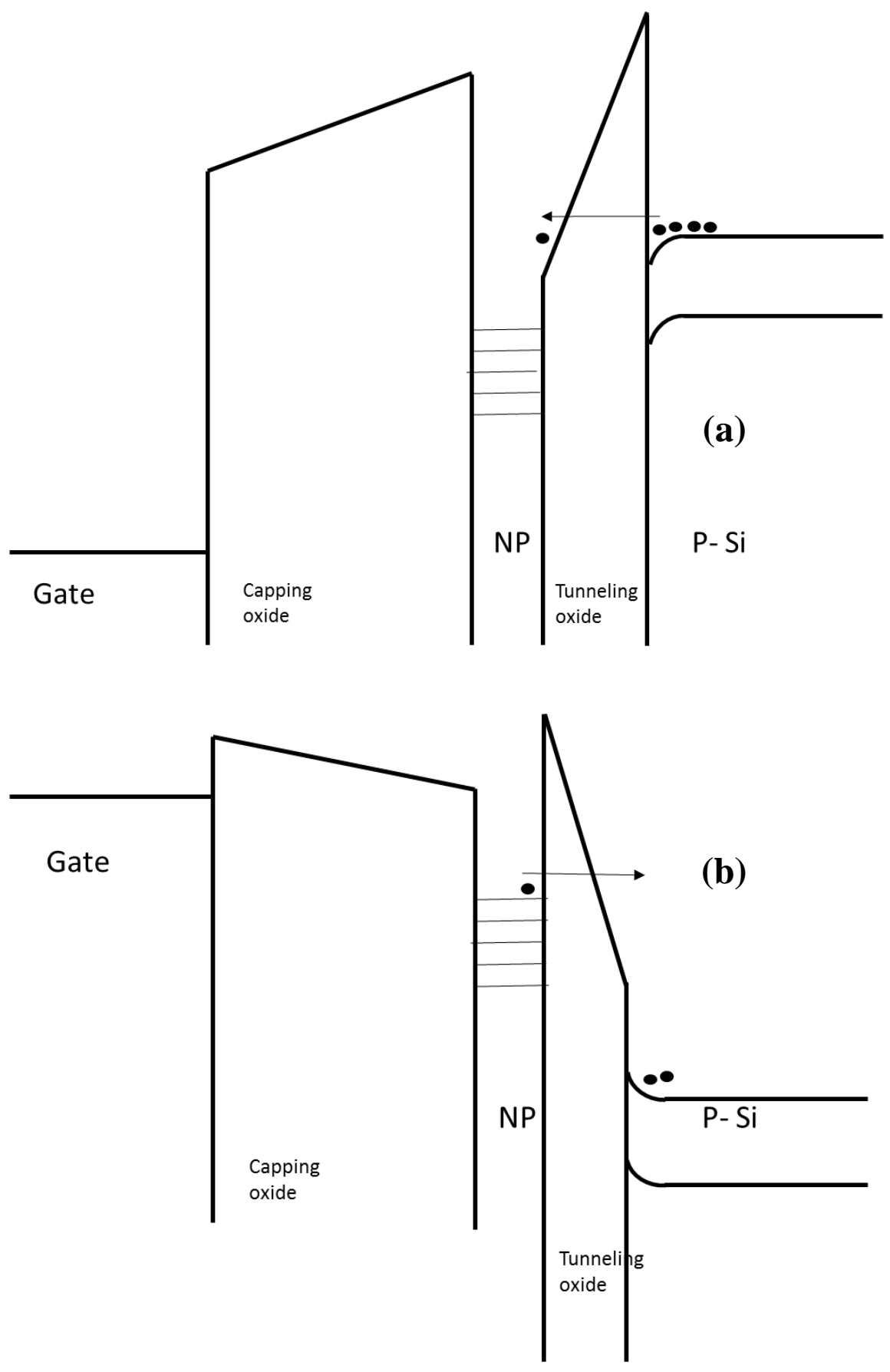

Figure 3-1: Energy band diagram FN tunneling for (a) Programming and (b) Erasing 


\subsubsection{Retention Characteristics}

When a nonvolatile memory cell can no longer hold the charge in the floating gate or metal NP, it is said to have affected its retention capability. Retention is a measure of the time that a nonvolatile memory cell can retain the charge whether it is biased or not. In nanoparticle discrete memories, the stored charge can leak away from the floating gate through the gate oxide or through tunneling oxide. This leakage can be caused by mobile ions and oxide defects and results in a shift of the threshold voltage of the memory device. Different charge loss mechanisms have been described [29, 30], namely, charge loss due to thermionic emission(at room temperature), charge loss due to electron detrapping, and charge loss due to contaminations such as positive mobile ions. To improve the retention characteristics of the memory cell, various improvements to the quality of the gate oxide, tunneling oxide and size and structure of the floating gate metal NP become very important. Retention can be quantified by measuring or estimating the time it takes for the floating gate NP to discharge when it is intended to keep the information stored. Typically, retention can be improved significantly by modifying the Coulomb charging and Quantum confinement energy of the floating gate used.

\subsubsection{Characteristic charging energies}

Charging a nanoparticle with electrons are often associated with two kinds of energies: Coulomb charging energy and Quantum confinement energy, where the Coulomb charging energy arises from the self-capacitance of the NP and the Quantum confinement is linked to the spatial confinement of electrons[1]. 
Coulomb charging energy is based on electrostatic capacitive charging energy, where for every addition charge $d q$ transported to a conductor, work has to be done against the field of already present charges. Thus charging a capacitor with a charge q required

$$
E=(q)^{2} / 2 C
$$

Where $\mathrm{C}$ is the total capacitance coupled to the NP, and this changes according to the NP size, density, tunneling and blocking oxide thickness and number of NP layers in the system.

For ultra-small MNPs, self-capacitance $(C)$ is calculated by:

$$
C=4 \pi \varepsilon_{\text {medium }} a\left[1+\frac{a}{2 d}+\frac{\left(\frac{a}{2 d}\right)^{2}}{1-\left(\frac{a}{2 d}\right)^{2}}\right]
$$

where $\varepsilon_{\text {medium }}$ is the permittivity of the surrounding medium $\left(8 \varepsilon_{0}\right.$ for $\left.\mathrm{Al}_{2} \mathrm{O}_{3}\right), a$ is the MNP radius, and $d$ is the distance between the center of the MNP and the substrate surface[26].

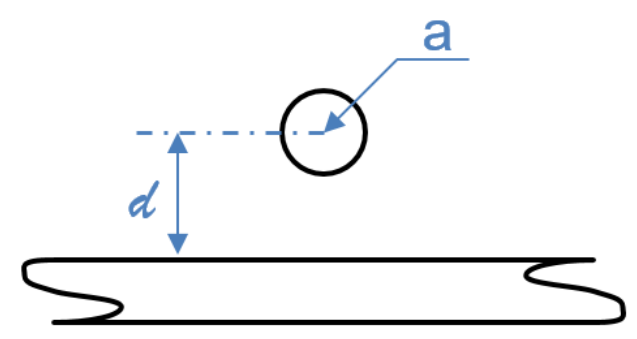

The corresponding Coulomb charging energy $(E)$ is given as:

$$
E=(n q)^{2} / 2 C
$$


where $q$ is the elementary charge. Note that varying the particle number density modifies the interparticle distance $(l)$ and, thus, its Coulomb charging energy. The calculated Coulomb charging energy values for PtNPs used in this work are summarized in Table 3-3. For $0.52 \pm 0.12 \mathrm{~nm} \mathrm{PtNP}\left(23.8^{\circ}\right.$ target angle and $5 \mathrm{~s}$ sputtering time), $E$ was calculated to be $336 \mathrm{meV}$, which is the highest reported so far with MNPs and has also allowed for room temperature observation of single electron tunneling [19, 33]. Furthermore, since Coulomb charging energy corresponds to the energy required to add or remove one electron from the PtNP, a high value for $E$ indicates the ability to hold a charge strongly improving the device retention properties.

On the other hand, Quantum confinement energy is based on the energy level spacing, where with decreasing NP sie the energy level spacing of electron states increases indirectly proportional to the NP size[1].

$$
\text { Energy level Spacing } \frac{d E f}{d N}=\frac{2}{3} \frac{E f}{N}
$$

Where $\mathrm{E} f$ is the Fermi-energy of the bulk material and $\mathrm{N}$ is the number of atoms available in the NP. Quantum confinement energy for different sized Pt NP is shown in Table 3-1.

Table 3-1: Quantum confinement energy for different Pt NP
$\begin{array}{cc}\text { NP size } & \text { QCE(meV) } \\ 0.5 & 663.3333 \\ 0.7 & 234.1177 \\ 1.11 & 57.68116 \\ 0.65 & 284.2857\end{array}$


The sum of Coulomb charging energy and the Quantum confinement energy defines the energy required to charge/discharge the nanoparticle in consideration.

\subsection{Experimental Section}

\subsubsection{Device Preparation}

Figure 3-6(a) shows the schematic of the device structure. The samples were prepared on Shiraki-cleaned P-type Silicon wafers (Float zone grown) with 5-10 $\Omega^{-1}-\mathrm{cm}^{-1}$ resistivity. Tunneling and interparticle $\mathrm{Al}_{2} \mathrm{O}_{3}$ dielectric layers were deposited by atomic layer deposition (ALD, Cambridge Nanotech) at $300 \quad{ }^{\circ} \mathrm{C}$ using water and Trimethylaluminum (TMA) precursors with a deposition rate of $0.8 \AA$ Ácycle. Since hydroxyl-terminated surfaces are essential for complete TMA reaction coverage and defect-free $\mathrm{Al}_{2} \mathrm{O}_{3}$ formation[31], water was pulsed for twenty cycles (0.02 s pulses with 8 s purge interval) prior to flowing any TMA vapor. This was followed by repetitive cycles of $0.015 \mathrm{~s}$ TMA and $0.02 \mathrm{~s} \mathrm{H}_{2} \mathrm{O}$ with $8 \mathrm{~s}$ purge interval. The numbers of ALD cycles required for each thickness are provided in Table 3-2, with specific thicknesses measured using a J.A. Woollam ellipsometer (VASE).

For ellipsometry measurement, a $50 \mathrm{~nm} \mathrm{Al}_{2} \mathrm{O}_{3}$ (actual $53.28 \pm 0.056 \mathrm{~nm}$ ) control was first fabricated and used to determine the optical constants for the ALD process. These measured optical constants were than kept fixed to that of standard alumina to determine the thickness of $\mathrm{Al}_{2} \mathrm{O}_{3}$ less than $20 \mathrm{~nm}$ using the Cauchy model. After the tunneling layer deposition, the first Platinum nanoparticle (PtNP) layer was deposited using a tilted target configuration with varying target angle and $30 \mathrm{~W}$ deposition power as detailed previously[32]. This was followed by depositing an interparticle $\mathrm{Al}_{2} \mathrm{O}_{3}$ layer at $100^{\circ} \mathrm{C}$. The 
second layer PtNP was then deposited with different deposition parameters. This was succeeded by a $15 \mathrm{~nm} \mathrm{Al} \mathrm{O}_{3}$ capping layer and $1 \mathrm{hr}$ Hydrogen anneal at $260{ }^{\circ} \mathrm{C}$ [21]. Finally, a $150 \mathrm{~nm}$ Titanium gate was deposited using a Kurt J. Lesker Axxis electron beam evaporator.

Table 3-2: Thickness and ALD process parameters for each $\mathrm{Al}_{2} \mathrm{O}_{3}$ layer

\begin{tabular}{|c|c|c|c|}
\hline \multirow{2}{*}{ Type of Layer } & \multicolumn{1}{|c|}{$\begin{array}{l}\text { Thickness } \\
(\mathbf{n m})\end{array}$} & $\begin{array}{l}\text { \# } \text { of } \\
\text { Cycles }\end{array}$ & Temperature \\
\hline \multirow{2}{*}{ Tunneling } & $1.9 \pm 0.001$ & 25 & $300^{\circ} \mathrm{C}$ \\
\cline { 2 - 4 } & $3.18 \pm 0.008$ & 35 & $300^{\circ} \mathrm{C}$ \\
\hline Interparticle & $3.22 \pm 0.003$ & 35 & $100^{\circ} \mathrm{C}$ \\
\hline Capping & $14.91 \pm 0.0071$ & 162 & $100^{\circ} \mathrm{C}$ \\
\hline
\end{tabular}

Different combinations of Pt nanoparticle size and number density were chosen from our previous publications for the first and second PtNP layers (Table 3-3) [21]. For each configuration, control samples were fabricated either without PtNP or with only a single PtNP layer deposited on different thicknesses of $\mathrm{Al}_{2} \mathrm{O}_{3}$ to simulate the multi-layer structures without either the first or second PtNP layer and demonstrate that the step-like $V_{\mathrm{FB}}$ charging effects are unique to the multi-layer device configurations. 
Table 3-3: Pt Nanoparticle Size and Density Configuration at Different Layers

\begin{tabular}{|c|c|c|c|c|c|c|}
\hline $\begin{array}{l}\text { Layer } \\
\text { Type }\end{array}$ & $\begin{array}{l}\text { Target } \\
\text { Angle }\end{array}$ & $\begin{array}{c}\text { Depo } \\
\text { sition } \\
\text { Time } \\
\text { (s) }\end{array}$ & Mean Diameter (nm) & $\begin{array}{l}\text { Number } \\
\text { Density }\end{array}$ & $\begin{array}{l}\text { Interparticle } \\
\text { Distance (nm) }\end{array}$ & $\begin{array}{c}\text { Theoret } \\
\text { ical } \\
\text { Coulom } \\
\text { b } \\
\text { Chargin } \\
\text { g } \\
\text { Energy } \\
\text { (meV) }\end{array}$ \\
\hline First & $23.8^{\circ}$ & 5 & $0.52 \pm 0.12$ & $1.7 \times 10^{12} / \mathrm{cm}^{2}$ & $4.65 \pm 2.09$ & 336 \\
\hline First & $38.8^{\circ}$ & 20 & $0.7 \pm 0.19$ & $10 \times 10^{12} / \mathrm{cm}^{2}$ & $1.92 \pm 0.54$ & 238 \\
\hline Second & $23.8^{\circ}$ & 20 & $1.11 \pm 0.28$ & $5.4 \times 10^{12} / \mathrm{cm}^{2}$ & $2.75 \pm 1.05$ & 147 \\
\hline
\end{tabular}

\subsubsection{Electrical characterization:}

Capacitance-Voltage (CV) measurements were performed at room temperature in a 4200SCS Keithley semiconductor characterization instrument. CV measurements were taken at $1 \mathrm{MHZ}$ in a voltage sweep range up to $\pm 18 \mathrm{~V}$. The voltage was swept initially with $\pm 1 \mathrm{~V}$ to demonstrate the absence of initial PtNP charging in the dielectric stack. A shielded probe station from Signatone micromanipulators was used as a testing station.

\subsubsection{Particle deposition and TEM characterization:}

For transmission electron microscopy (TEM) image analysis of the multi-layer stack, the same device stack was prepared without the Ti electrode on Carbon grids (500 mesh $\mathrm{Cu}$ ) and Silicon substrates. The Carbon grids were used for analysis using the plane view images and the Silicon substrates were used to prepare for cross-sectional TEM imaging. The low electron density of amorphous $\mathrm{Al}_{2} \mathrm{O}_{3}$ was favorable to characterize the size and density of these particles using Top-view TEM viewing. The Fork and tube method was implemented for sample preparation of the cross sectional sample, details of which are mentioned in [20]. Further characterization involved acquiring the Pt nanoparticles size and distribution for plane view image from JEOL 1400 TEM and for 
cross sectional image from Technai F20 high resolution TEM. EDS spectrum was also obtained using a Technai F20 TEM with EDS capability.

Figure 3-2(a) shows the plane view TEM image of a single-layer of uniformly distributed $1.08 \mathrm{~nm}$ Pt nanoparticles where the density was found to be $6.6 \times 10^{12} \mathrm{~cm}^{-2}$. The resulting nanoparticles are spherical in shape having near uniform particle size and a good separation of $2.75 \mathrm{~nm}$ from each other. Figure 3-2 (b),(c) and (d) shows the plane view image for double, triple and quadruple layers of Pt nanoparticles separated by $3 \mathrm{~nm}$ $\mathrm{Al}_{2} \mathrm{O}_{3}$ spacer layer, where the density of $\mathrm{Pt}$ nanoparticle increases with number of layers without a change in mean diameter. Though, Pt nanoparticle density variations can be determined from the image analysis, the actual density prediction (calculated by multiplying number of layers to density of a single layer Pt nanoparticle) in a device stack could not be compared with the observed density using image analysis. Here, since the plane view TEM image is a top view of the multilayer, there will be instances where the particle might lie under another particle from a consecutive/alternative layer. Since TEM is based on electron density, the projected image will show such overlapping particles as one which will account for a lower density value from image analysis. Thus for multi-layer stacked on Si-based devices, the density will multiply with increasing layers unlike what was calculated from image analysis using top view TEM images. 

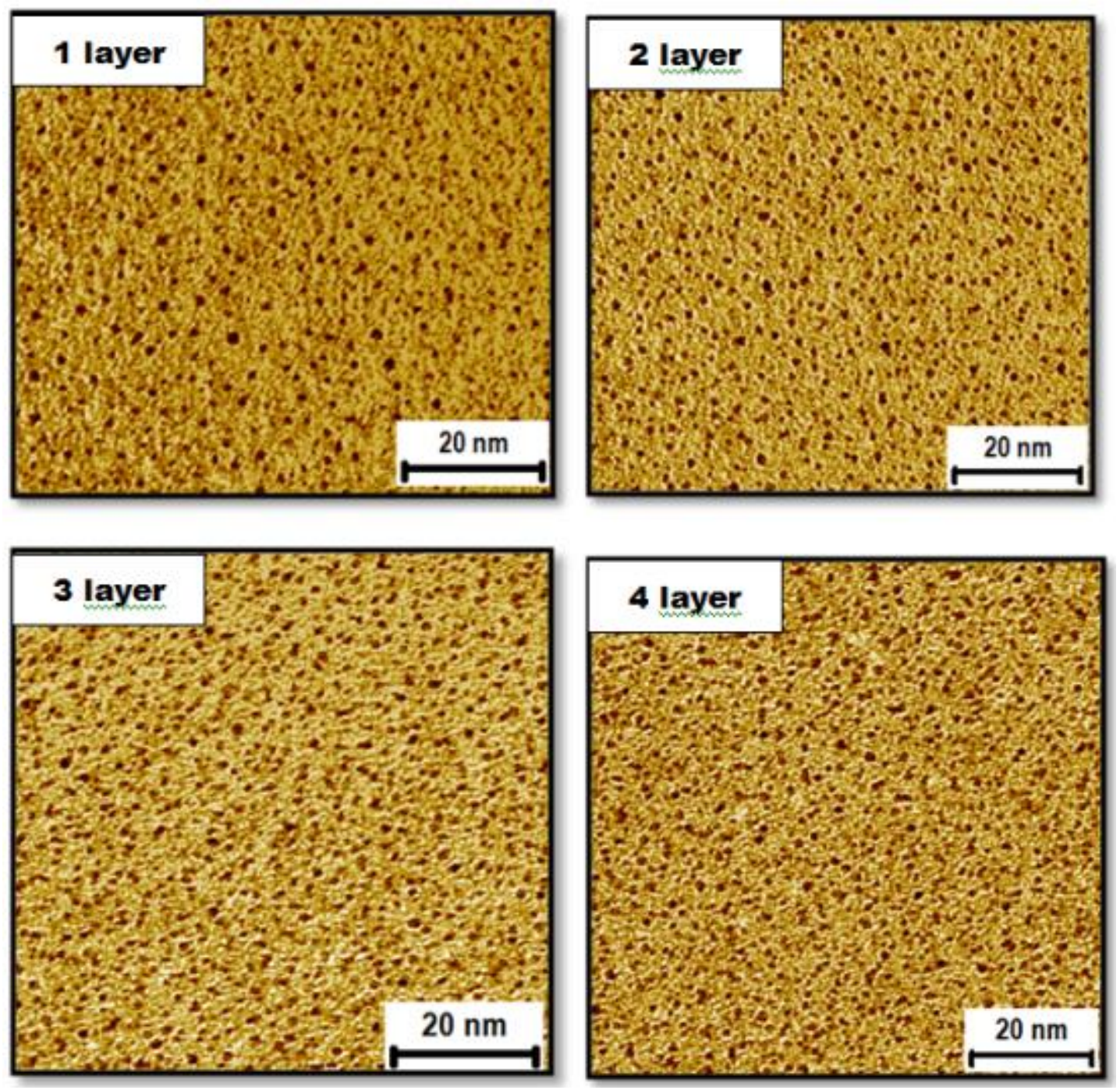

Figure 3-2: (a) Plane view TEM image of a single-layer of uniformly distributed $1.08 \mathrm{~nm} \mathrm{Pt}$ nanoparticles. The inset of (a) shows particle size distribution over 5 images after statistical postimage analysis (b) Plane view TEM for double layer Pt nanoparticles (c) Plane view TEM for triple layer Pt nanoparticles. (d) Plane view TEM for quadruple layer Pt nanoparticles 

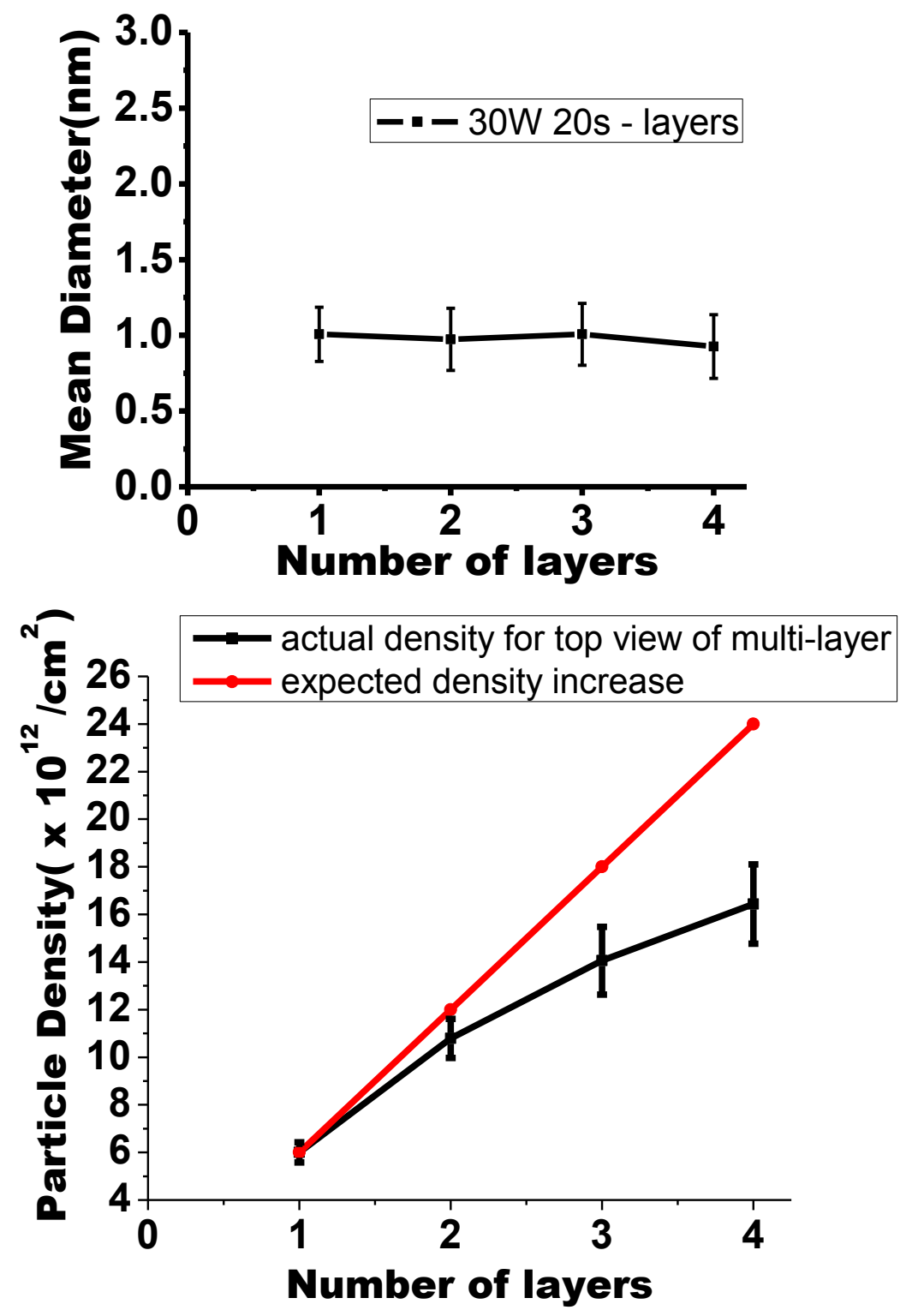

Figure 3-3 Plots from image analysis of multiple layers showing (a) Mean Diameter vs. Number of Layers and (b) Particle Density vs. Number of Layers. 
Figure 3-3 shows the plots for mean particle diameter and particle density for different number of Pt NP layers from the image analysis. It was found that the mean diameter is almost constant with varying number of layers but the density increases with increase in number of layers. Thus, from the observed increase in particle density without any change in mean diameter, we can derive that the Pt nanoparticles are not coagulating during the multilayer fabrication process and hence it retains its size when incorporated in multiple layers separated by a $3 \mathrm{~nm} \mathrm{Al}_{2} \mathrm{O}_{3}$ layer.

The existence of Pt nanoparticles was further examined using EDS spectrum to determine the Pt density variation with multilayer stacking. Figure 3-4(a-d) shows the elemental mapping for these grids using EDS analysis. It is clear that the intensity peak for Pt increases as we increase the number of layers. Figure 3-4(e) shows the integrated intensity plot for Pt at the M-shell and L-shell excitation levels. The consistent increase in the intensity also proves the existence of Pt NP layers. 

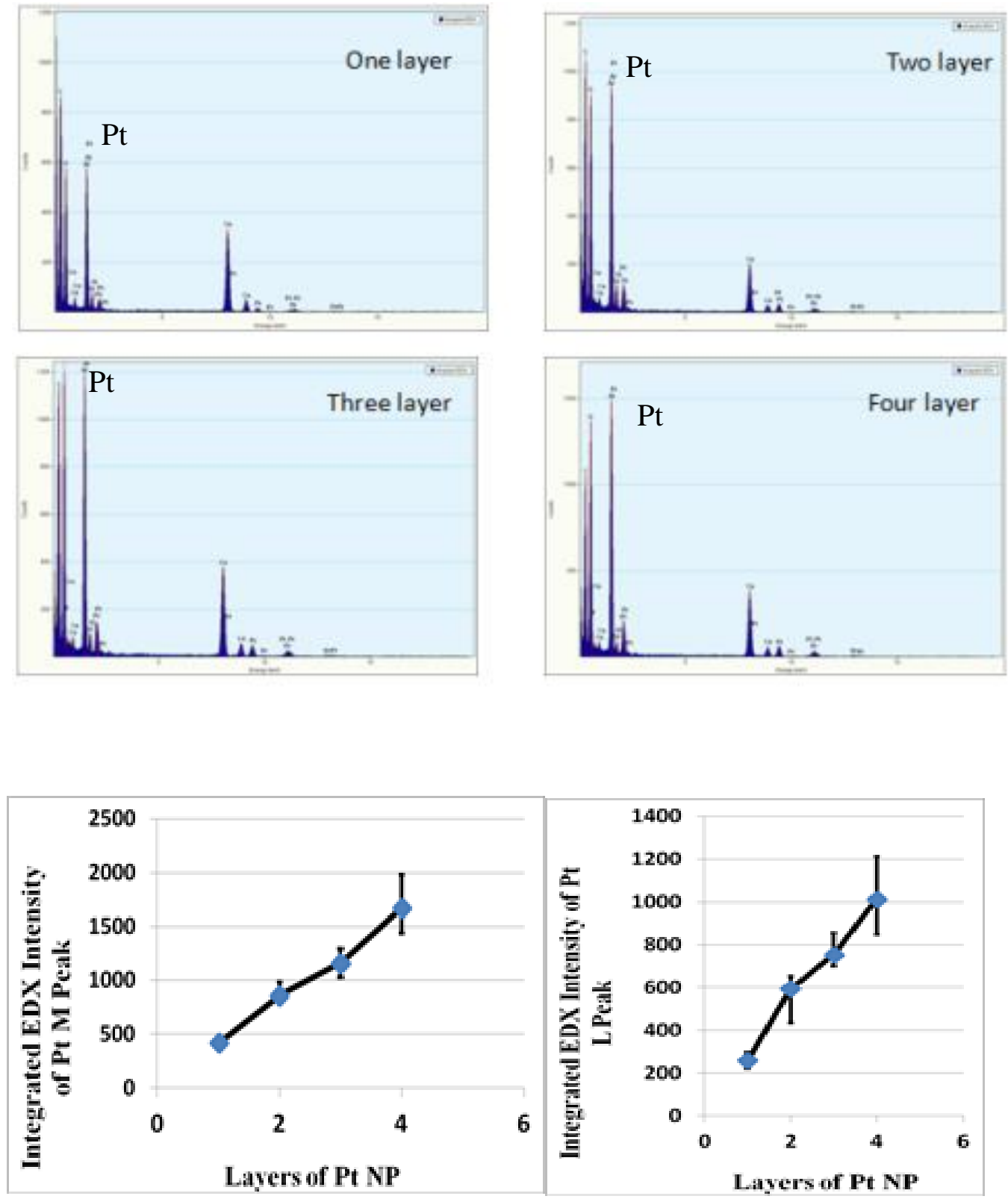

Figure 3-4: EDS Spectrum of (a) single-, (b) double-, (c) triple-, and (d) quad-layer Pt nanoparticle samples. (e) Integrated intensity of $\mathrm{Pt} M$ peak (f) Integrated intensity of $\mathrm{Pt} L$ peak 
Figure 3-5 shows the cross sectional high resolution TEM image of the multi-layer Pt nanoparticles. A major problem faced during this TEM imaging was the coalescence and collapsing of multi-layer Pt nanoparticles due to the electron dose. Thus a process was adopted where the image was taken as soon as the beam was introduced to the specimen; this resulted in images having less contrast but without any beam-induced coalescence. The inset of Figure 3-5 (b) shows the size distribution of these nanoparticles. The mean diameter is around $\sim 1.1 \mathrm{~nm}$ which is close to the mean of $1.08 \mathrm{~nm}$ from plane view image. The inter-particle layer analysis also showed a mean value of $3.14 \mathrm{~nm}$ proving the presence of multi-layer Pt nanoparticles which were analyzed by measuring vertical distances between nanoparticles. Thus, any beam induced coalescence was avoided during TEM characterization.

This work on quad-layer stacks showed the survival of PtNPs when stacked on different numbers of layers with a $3 \mathrm{~nm}$ oxide separation layer [20]. Although different NP size and density configurations were used in these experiments, we assumed that PtNP size and density were not affected through addition of multiple layers provided there was at least $3 \mathrm{~nm}$ separation layer between different PtNP layers. 


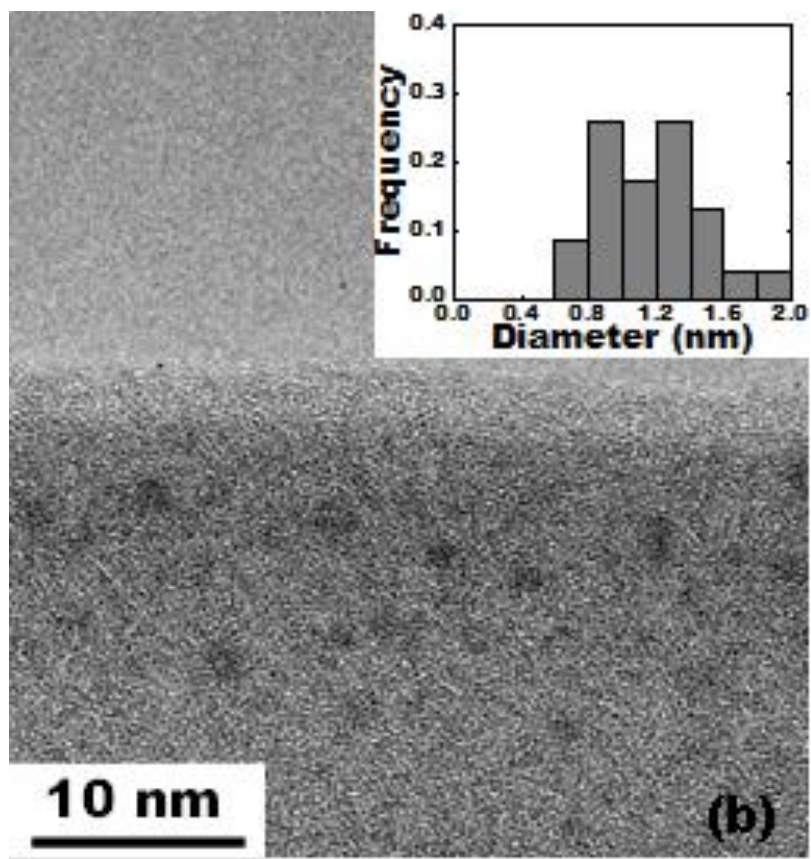

Figure 3-5: Cross-sectional TEM of quad layer sample. Inset shows the particle size distribution after statistical post-image analysis 


\subsection{Results and Discussions}

\subsubsection{Pt Nanoparticle Configuration}

The tilted target configuration allows tunable variation in the in-flight metal atom density interacting with the substrate by changing the focal point of the deposition flux with time. The change in flux modifies the metal thermalization at the surface, which in turn affects the nanoparticle size and number density[32]. The type of process governing the MNP growth can be alternated by using either high energy for MNP coalescence or low energy for random nucleation. Through precise tuning of each of the deposition parameters, ultra-fine MNPs can be produced with specific average size, particle number density, and homogenous size distribution [32]. Figure 3-6(b-d) shows the plane view TEM images for PtNP deposited by tilted target configuration at tilt angle/deposition times of $23.8^{\circ} / 5 \mathrm{~s}(0.52 \pm 0.12 \mathrm{~nm}), 23.8^{\circ} / 20 \mathrm{~s}(1.11 \pm 0.28 \mathrm{~nm})$, and $38.8^{\circ} / 20 \mathrm{~s}(0.7 \pm 0.19 \mathrm{~nm})$, respectively. The $0.52 \pm 0.12 \mathrm{~nm}$ PtNPs (Figure 1b) were used for the primary MNP layer. The second MNP layer was deposited with a different particle size and number density following Figure 3-6 (c,d) as mentioned in Table 3-3. 

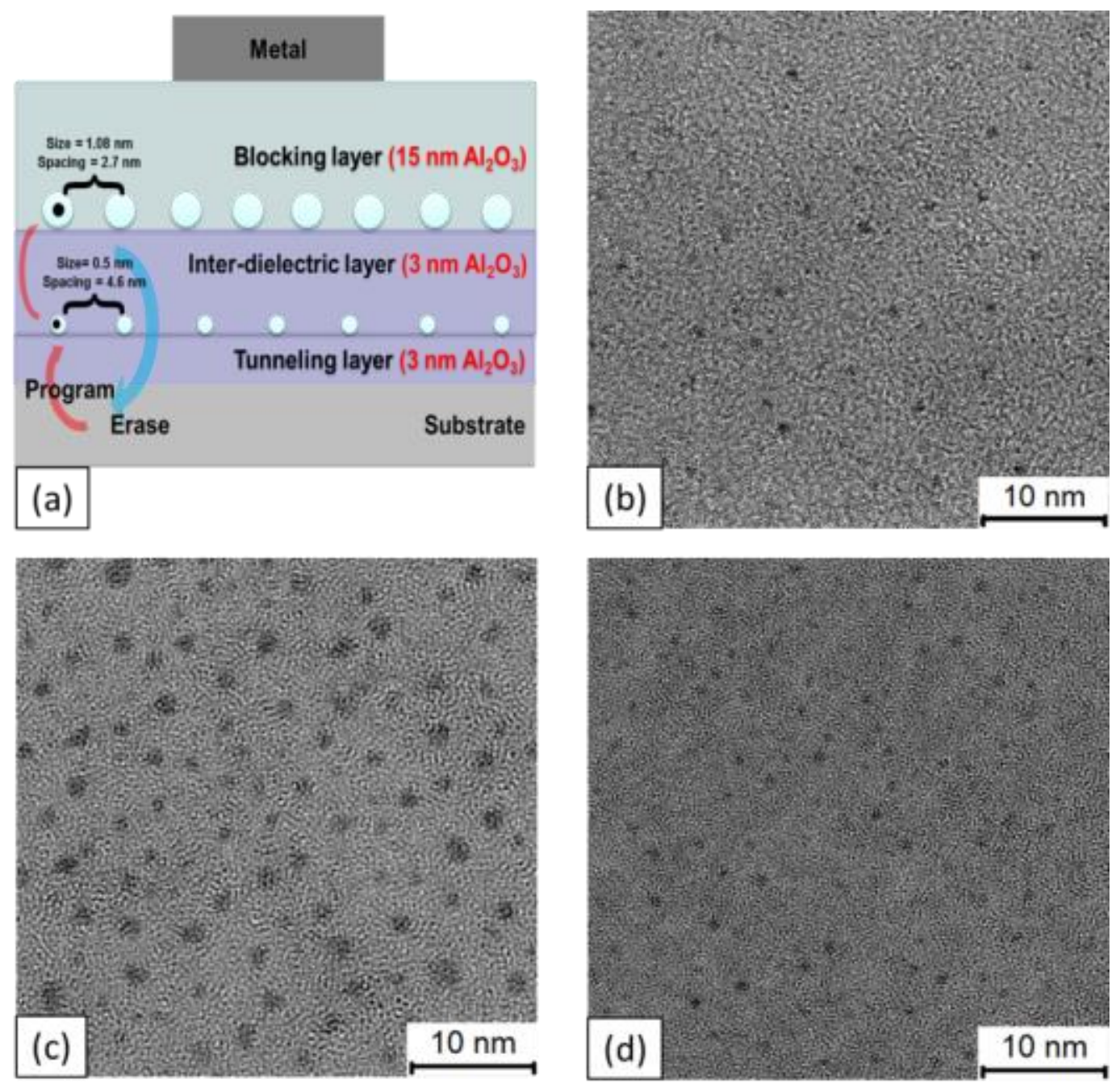

Figure 3-6:(a) Schematic of double-layer NVM MOSCAP device designed for controlled charging. Plane view HRTEM for (b) $0.52 \pm 0.12 \mathrm{~nm}$ PtNP $\left(23.8^{\circ} / 5 \mathrm{~s}\right)$, (c) $1.11 \pm 0.28 \mathrm{~nm} \operatorname{PtNP}\left(23.8^{\circ} / 20 \mathrm{~s}\right)$, and (d) $0.7 \pm 0.19 \mathrm{~nm}$ PtNP $\left(16.2^{\circ} / 20 \mathrm{~s}\right)$ 


\subsubsection{Demonstrating Controlled Nanoparticle Layer Charging through Capacitance-Voltage Measurement}

Figure 3-7 shows the Capacitance-Voltage (CV) measurements for single- and double-layer PtNP-embedded NVM MOSCAPs. In order to demonstrate controlled charging of each PtNP layer in the double-layer device and, thus, multi-bit feasibility, it is necessary to observe a step effect in $\Delta \mathrm{V}_{\mathrm{FB}}$ (i.e. the memory window) in the final device stack. Figure 3-7 (a) shows the negligible hysteresis of a control MOSCAP without PtNP, ensuring that $\mathrm{ALD}$-grown $\mathrm{Al}_{2} \mathrm{O}_{3}$ does not contribute significantly to MNP charging or hysteresis. All other PtNP-embedded devices were first scanned in a narrow range of $\pm 1 \mathrm{~V}$ to identify the minimum hysteresis region, indicating only $0.08 \mathrm{~V}$ charging of the PtNPs with electrons [21]. The counter-clockwise hysteresis loops indicate electron injection from substrate to PtNP under positive voltage for programming operation by FowlerNordheim Tunneling [34]. Figure 3-7(b) shows the CV for the MOSCAP embedded with only one layer of PtNP with $3 \mathrm{~nm}$ tunneling and $15 \mathrm{~nm}$ capping layers, fabricated to observe saturation of the memory window with respect to the bias. Saturation of $\Delta \mathrm{V}_{\mathrm{FB}}$ occurred beyond a program voltage of $\pm 7 \mathrm{~V}$, which can be explained by the limited electron capturing capacity of the $0.52 \pm 0.12 \mathrm{~nm}$ PtNP layer as a result of its low number density. Using the relationship $N_{t}=\left(C_{o x} \Delta \mathrm{V}_{\mathrm{FB}}\right) / q$, where $N_{t}$ is the trapped charge, $C_{o x}$ is the accumulation capacitance density of the oxide, and $q$ the elementary charge, the corresponding electron charge density was calculated to be $3.08 \times 10^{12} / \mathrm{cm}^{2}$. Recalling the PtNP number density of $1.72 \times 10^{12} / \mathrm{cm}^{2}$ (Table 3-3), the above charge density corresponds to charging of $\sim 1.79$ electrons per PtNP. This value is known to be a slight overestimation since the PtNP number density is undoubtedly underestimated as a result of the inherent 
limitations to TEM characterization of the PtNPs[19, 32]. Thus, the electron charging per PtNP is estimated to be about one.

To simulate the activity of the second PtNP layer in a dual-layer PtNP-embedded MOSCAP, a device was fabricated with similar configuration to the dual-layer device but without the first PtNP layer. A $3 \mathrm{~nm}$ tunnel oxide described in Table 3-2 followed by a 3 $\mathrm{nm}$ interparticle was deposited resulting in a total of $6 \mathrm{~nm}$ tunneling oxide. PtNP with $\sim 1.11$ $\pm 0.28 \mathrm{~nm}$ diameter and $5.4 \times 10^{12} / \mathrm{cm}^{2}$ particle number density were deposited over this thicker tunneling oxide $(\sim 6.4 \mathrm{~nm})$ Figure 3-7 (c) shows the CV characterization for this device. Minimum initial charging was observed for this device even up to a programming voltage of $\pm 15 \mathrm{~V}$. Above $15 \mathrm{~V}$, PtNP charging began and a distinct memory window was observed. The saturation of the memory window could not be reached for this device as the thin capping oxide thickness of $15 \mathrm{~nm}$ limited the measurements to a maximum bias of $18 \mathrm{~V}$, beyond which device breakdown would occur. These observations lead to an expectation of controlled charging of the PtNP layer when these two PtNP layer configurations are combined to form the final double-layer NVM MOSCAP.

Figure 3-7 (d) shows the CV measurements for the final double-layer MOSCAP device fabricated combining the devices from Figure 3-7 (b) and (c) (i.e. $3 \mathrm{~nm}$ tunneling $\mathrm{Al}_{2} \mathrm{O}_{3}$ layer, $0.52 \pm 0.12 \mathrm{~nm}$ primary PtNP layer, $3 \mathrm{~nm}$ interparticle $\mathrm{Al}_{2} \mathrm{O}_{3}$ layer, $1.11 \pm$ $0.28 \mathrm{~nm}$ second PtNP layer, and $15 \mathrm{~nm}$ blocking $\mathrm{Al}_{2} \mathrm{O}_{3}$ layer). This device stack was probed over a voltage sweep range of $\pm 18 \mathrm{~V}$. There was an apparent increase in memory window associated with increase in bias, shifting the $\mathrm{V}_{\mathrm{FB}}$ in both directions and saturating over a voltage range of 7 to $14 \mathrm{~V}$ in both sweep directions. The near constant flat band voltage from $7 \mathrm{~V}$ up to a bias of $14 \mathrm{~V}$ indicates complete charging of the first PtNP layer 
at $7 \mathrm{~V}$ with a charge density of $4.2 \times 10^{12} / \mathrm{cm}^{2}$. $\mathrm{V}_{\mathrm{FB}}$ then shifts again beyond this range, exhibiting an increase in memory window (see also Figure 3-7 (e-f)). This shows that for a $6 \mathrm{~nm}$ tunneling oxide, there is no direct charging from the substrate to the second layer PtNPs until a $14 \mathrm{~V}$ bias is applied. Figure 3-7 (e) and (f) show the gate voltage dependence of the flat band voltage as extracted from the $\mathrm{CV}$ measurements for the program and erase operation and the total calculated memory window, respectively, which illustrate the distinct, controllable layer charging more explicitly than the raw CV measurements themselves. 

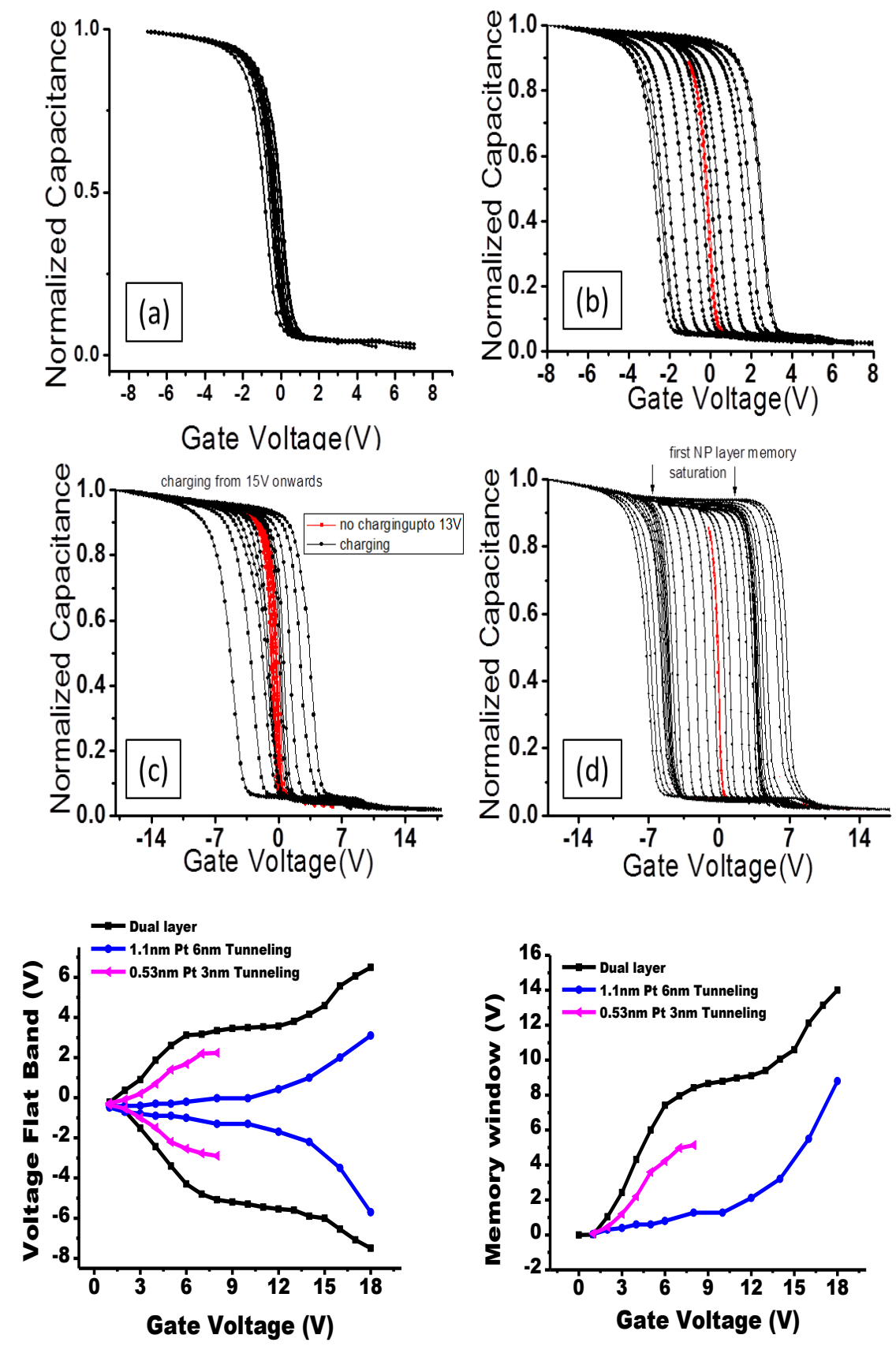

Figure 3-7: (a-d) CV characteristics for NVM MOSCAP with (a) no PtNP layer (control), (b) a single $0.52 \pm 0.12 \mathrm{~nm}$ PtNP layer over a $3 \mathrm{~nm}$ tunneling oxide layer, (c) a single $1.11 \pm 0.28 \mathrm{~nm}$ PtNP layer over a $6 \mathrm{~nm}$ tunneling oxide layer, (d) the final double-layer device $(3 \mathrm{~nm}$ tunneling layer $\mid 0.52 \pm 0.12$

$\mathrm{nm}$ PtNP layer | $3 \mathrm{~nm}$ separation oxide | $1.11 \pm 0.28 \mathrm{~nm}$ PtNP layer); (e) Flat band voltage as a function of gate voltage during program and erase conditions; and (f) Plot illustrating the step effect in the memory window as a function of gate voltage. 


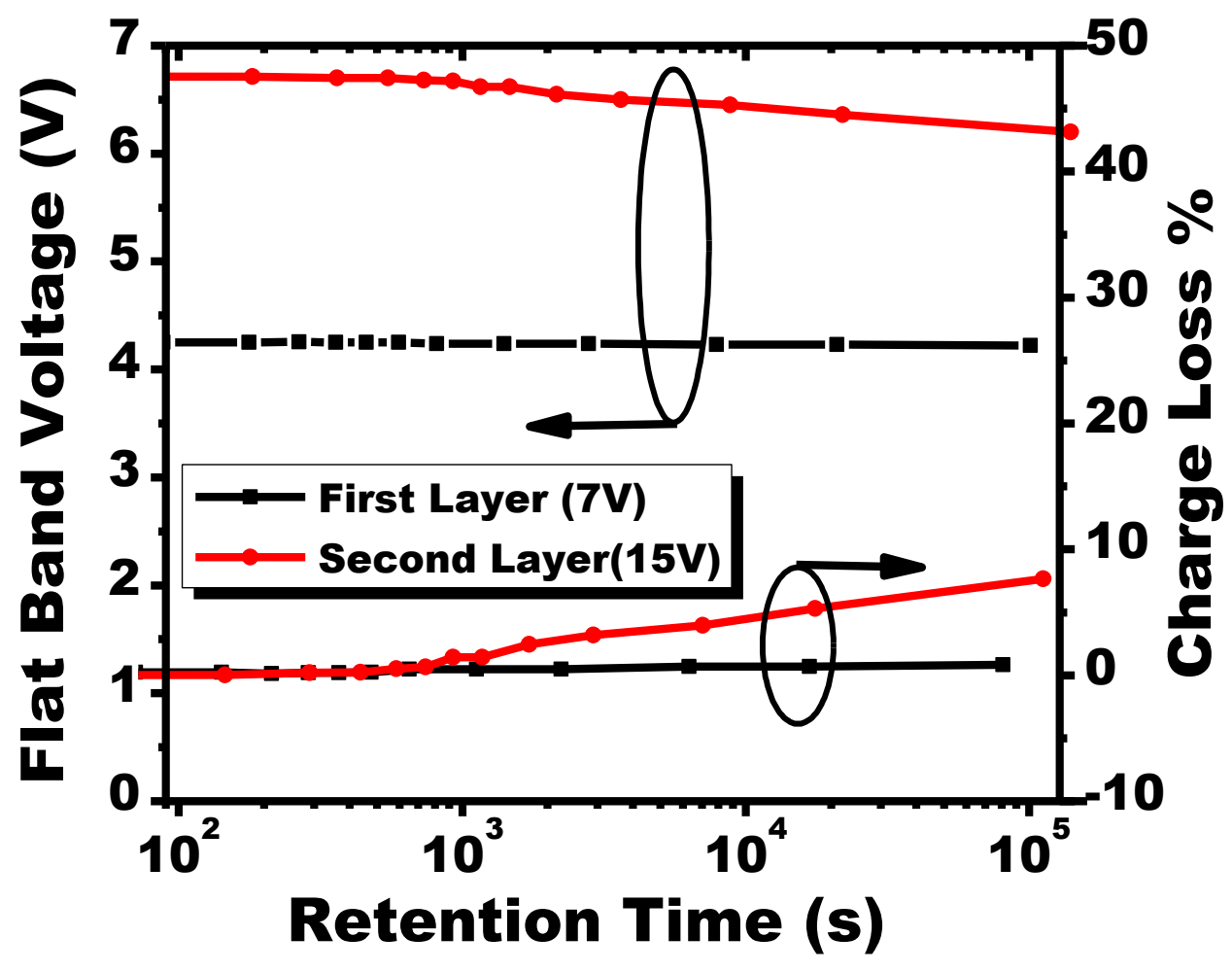

Figure 3-8 Retention characteristics for first layer charging and second layer charging with $7 \mathrm{~V}$ and $15 \mathrm{~V}$ respectively. The left axis denotes change in Flat Band and the right axis denotes the total charge loss in percentage. 
In order to check device reliability in terms of oxide leakage and NP configuration, we performed charge retention measurements for the dual layer device at $7 \mathrm{~V}$ and $15 \mathrm{~V}$ programming voltage. Figure 3-8 shows that whole device charge retention (both first and second layer charging) is extremely solid with less than $9 \%$ charge loss for over $10^{5} \mathrm{~s}$. Meanwhile, the charge retention for the first layer only is greater than $98 \%$ due to the higher Coulomb charging energy for $0.52 \pm 0.12 \mathrm{~nm}$ PtNPs. Thus, our devices can be used in multi-level architecture with good control over layer-by-layer charging, large memory window, and longer retention times.

\subsubsection{Effect of thinner tunneling layer and larger diameter first layer PtNP}

As mentioned above, the controlled charging phenomenon requires a precise, optimized nanoparticle configuration and device structure. Figure 3-9 shows the CV measurements of alternate double-layer device configurations with (a) a $2 \mathrm{~nm}$ tunneling oxide layer with the rest of the device configuration identical to that shown in Figure 3-9 (d) and (b) $0.7 \pm 0.19 \mathrm{~nm}$ diameter PtNPs with a smaller interparticle distance of $1.92 \mathrm{~nm}$ as the initial layer with the rest of the device configuration identical to that shown in Figure 3-7(d). From Figure 3-9 (a), it can be seen that devices with a $2 \mathrm{~nm}$ tunneling oxide layer resulted in a $0.89 \mathrm{~V}$ initial charging even with a voltage sweep of $\pm 1 \mathrm{~V}$, indicating uncontrolled electron tunneling and a charge leaky device. This hinders the ability to exhibit controlled layer-by-layer charging and a huge memory window of $11 \mathrm{~V}$ is obtained. Similarly, increasing the PtNP diameter from $0.52 \pm 0.12 \mathrm{~nm}$ to $0.7 \pm 0.19 \mathrm{~nm}$ gives a corresponding smaller interparticle distance of $1.92 \mathrm{~nm}$ and the Coulomb charging energy 
is reduced to $238 \mathrm{meV}$ (Figure 3-9 (b)). In turn, the reduced Coulomb charging energy decreases the charge storage capability and there is charge leakage back to the substrate, indicated by the change in slope of the CV curves shown in the black focus circles in Figure 3-9 (b)[20]. We also observe an initial charging of $1.32 \mathrm{~V}$ for a sweep voltage of $\pm 1 \mathrm{~V}$. Again, the charge leakage at the PtNPs makes it difficult to observe controlled, step-like charging behavior and the device exhibits large memory windows showing complete charging with tunneled charges.

The fact that these configurations do not exhibit layer-by-layer charging does not eliminate the possibility that they can. For example, using a $2 \mathrm{~nm}$ tunneling layer might require the MNPs in the first layer to have a higher Coulomb blockade effect to avoid charge leakage. Similarly, for larger size MNP with lower Coulomb charging energy, we might be able to exhibit layer-by-layer charging phenomena with thicker tunneling oxide. Obviously, when changing these parameters, the second layer MNP size and interparticle distance should also be modified in order to achieve proper device configuration for layerby-layer charging. 

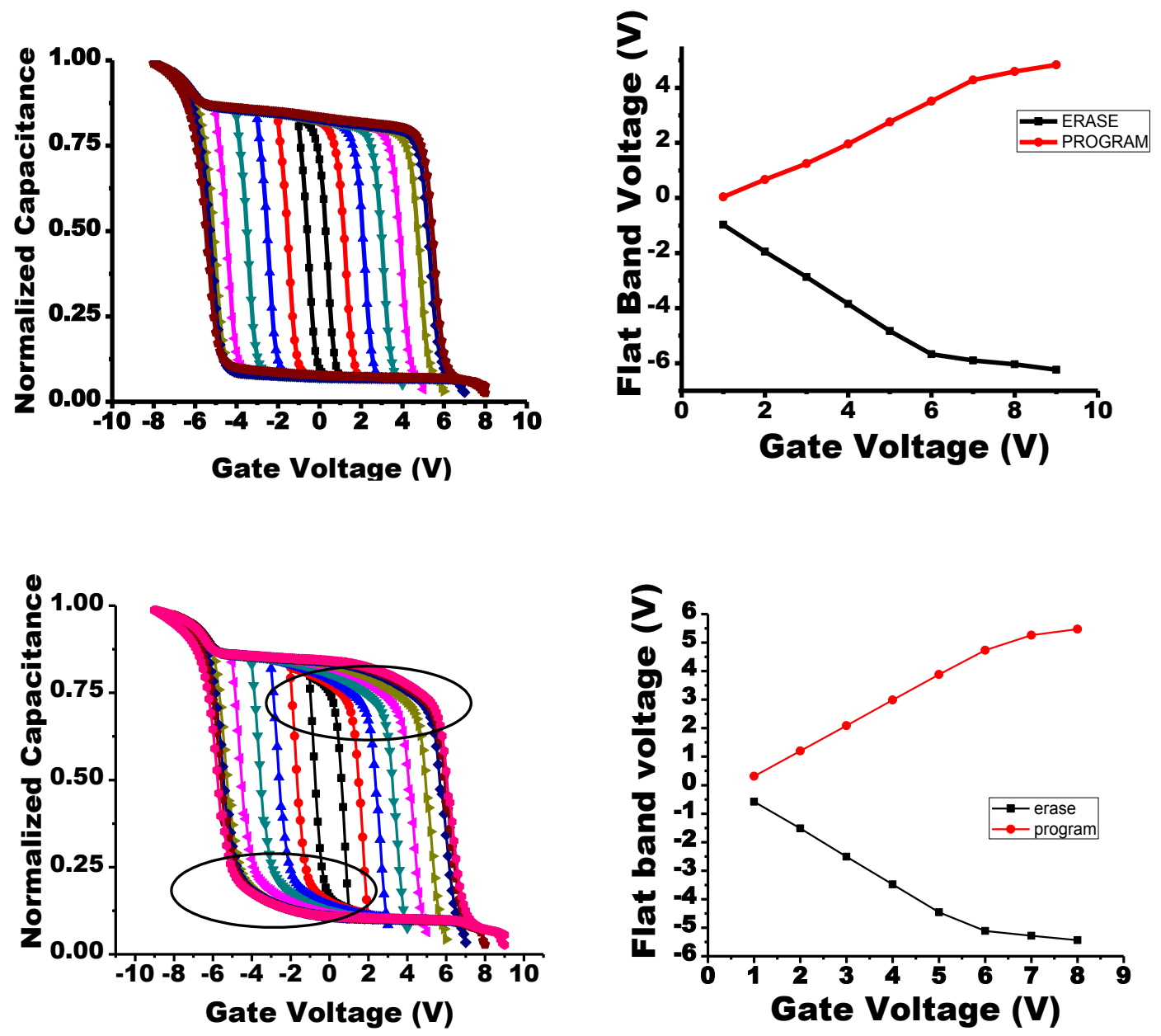

Figure 3-9: MOSCAP NVM with (a) $2 \mathrm{~nm}$ tunneling, $0.52 \pm 0.12 \mathrm{~nm}$ PtNP first layer, $3 \mathrm{~nm}$ interparticle layer thickness, $1.11 \pm 0.28 \mathrm{~nm}$ Second layer PtNP and $15 \mathrm{~nm}$ capping oxide (inset: extracted Flat band voltage vs. gate voltage for erase and programming mode); (b) $3 \mathrm{~nm}$ tunneling, $0.7 \pm 0.196 \mathrm{~nm}$ PtNP second layer, $3 \mathrm{~nm}$ interparticle layer thickness, $1.11 \pm 0.28 \mathrm{~nm}$ second layer PtNP and $15 \mathrm{~nm}$ capping oxide. (inset: extracted Flat band voltage vs. gate voltage for erase and programming mode) 


\subsubsection{Effect of varying the second layer Pt NP density}

Figure 3-10 shows the CV characteristics of MOSCAP NVM where the structure comprises of $3 \mathrm{~nm}$ tunneling $\mathrm{Al}_{2} \mathrm{O}_{3}$, first NP layer of $5 \mathrm{sec} \mathrm{Pt} \mathrm{NP}(\mathrm{TA}=23.8)$, 3nm separation $\mathrm{Al}_{2} \mathrm{O}_{3}$ layer and a low density Pt NP layer using $20 \mathrm{~s}(\mathrm{TA}=16.2 \mathrm{deg})$. Though there was negligible hysteresis window for $+-2 \mathrm{~V}$ of programming bias, the $\mathrm{Vfb}$ was shifted to difference of $+2 \mathrm{~V}$ from the ideal $\mathrm{Vfb}$ value. This shift corresponds to a total of $1.6 \mathrm{e} 12 / \mathrm{cm} 2$ charges using the relation for $\mathrm{N}_{\mathrm{t}}$ (trapped charge) A lower number density of the second layer Pt NP corresponding to a higher inter-particle distance, thereby increased the coulomb blockade energy associated with the nanoparticle. Due to this, some of the Pt NPs trapped the electrons in the bulk oxide and developed a partial charge inside the dielectric. Removing this electron from the Pt NP (embedded in the dielectric) becomes impossible using potential voltages resulting in a shift of the net $V_{f b}$ of the device. Because of this charging, from Figure 3-10 (a) the first NP layer is already saturated with electrons and the $\mathrm{Vfb}$ remains almost constant until a programming voltage of $+-15 \mathrm{~V}$. Beyond this, the charging of second Pt NP layer is shown, but here we can expect the second layer to saturate at an early programming voltage due to a lower particle number density of the Pt NP layer which corresponds to a lower programming efficiency and charge storage capacity. Since in this device configuration the first layer Pt NP was similar to the one used for devices in Figure 3-9 and only second layer Pt NP was varied, a control sample for second layer Pt NP was fabricated and tested. Pt NP with $1 \mathrm{~nm}$ diameter and $4 \mathrm{e} 12 / \mathrm{cm} 2$ particle density (20sec Pt NP/TA=16.2) were characterized with a thicker tunneling oxide of $6.5 \mathrm{~nm}$ between the Pt NP and Si substrate. Figure 3-10 (b) shows the CV characterization for this device with control second layer Pt NP MOSCAP without the first layer. It was observed 
that there was no initial charging for this device even up to a programming voltage of +$16 \mathrm{~V}$. Beyond $16 \mathrm{~V}$ the NP layer started charging and a memory window was observed. The saturation of the memory window could not be reached. Figure 3-10 (c) shows the extracted $\mathrm{V}_{\mathrm{fb}}$ values with respect to Programming and erasing operation for the $\mathrm{CV}$ plot in Figure 3-10 (a). It is clear that the step effect is visible only in the erase operation as the first layer NP are getting charged from the charge in the Pt particle even under no bias. Due to this the NP are stored with electron and saturates up to $15 \mathrm{~V}$. Beyond this there is increase in Vfb in both directions. For Figure 3-10 (d) the step effect is more prominent but ambiguous due to the reason that, the memory window is nothing but the difference of $\mathrm{Vfb}$ at programming and erasing, and for this particular case the step is purely due to effect of controlled erasing operation. 

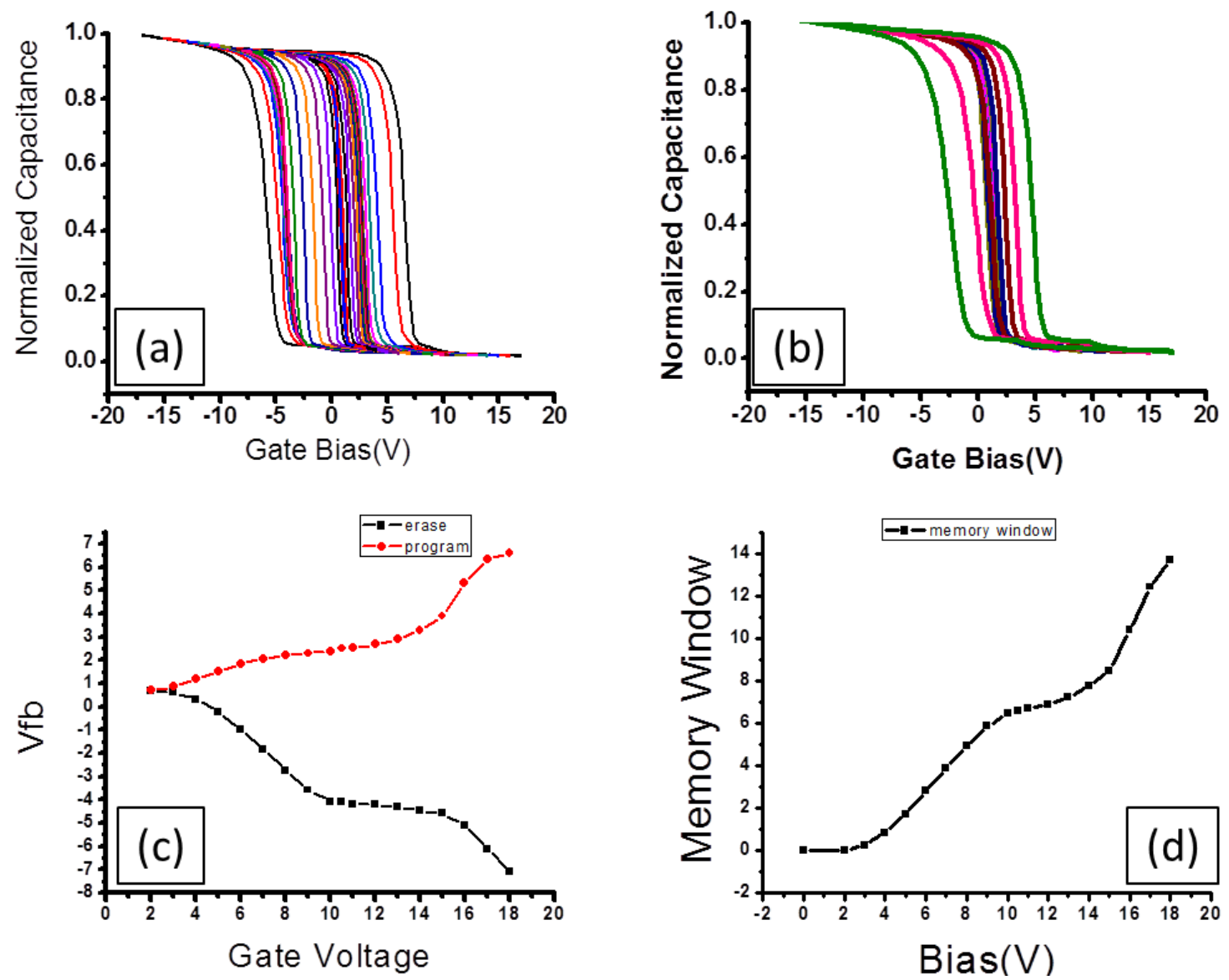

Figure 3-10: CV characteristics of MOSCAP NVM (a) dual layer Pt NP with 3nm tunneling $\mathrm{Al}_{2} \mathrm{O}_{3}$, first NP layer of 5 sec $\mathrm{Pt} N P(T A=23.8)$, 3nm separation $\mathrm{Al}_{2} \mathrm{O}_{3}$ layer and a low density $\mathrm{Pt}$ NP layer using 20s(TA=16.2 deg) and 15nm Capping oxide; (b) Second layer Pt NP (20s/TA=16.2) only with $7 \mathrm{~nm}$ tunneling and 15nm capping oxide; (c) Change in $V_{F B}$ for program and erase modes and; (d) Extracted memory window 


\subsubsection{Effect of a thicker separation layer}

Figure 3-11 shows the $\mathrm{CV}$ characteristics for a thicker separation of $4 \mathrm{~nm} \mathrm{Al}_{2} \mathrm{O}_{3}$ layer between the Pt NP. Figure 3-11(a) shows that due to an initial charging effect and a shift of $\mathrm{V}_{\mathrm{fb}}$ by $-0.4 \mathrm{~V}$, the initial charging was calculated to be $7.3 \mathrm{e} 11 / \mathrm{cm} 2$ but carried by holes (due to a negative shift) and the first Pt NP layer was partially charged with these holes. Thus from Figure 3-11 (b), we can see partial control over the erasing behavior, but due to depletion of charges, for a positive program voltage the majority of the electron population starts charging the first layer and saturation is not reached even with $+15 \mathrm{~V}$. At $15 \mathrm{~V}$ now the first layer Pt NP has enough energy to let the electron tunnel out from it to the second layer. Because of this effect, the device fails to exhibit a controlled charging or a step effect in its $V_{F B}$ plot. 

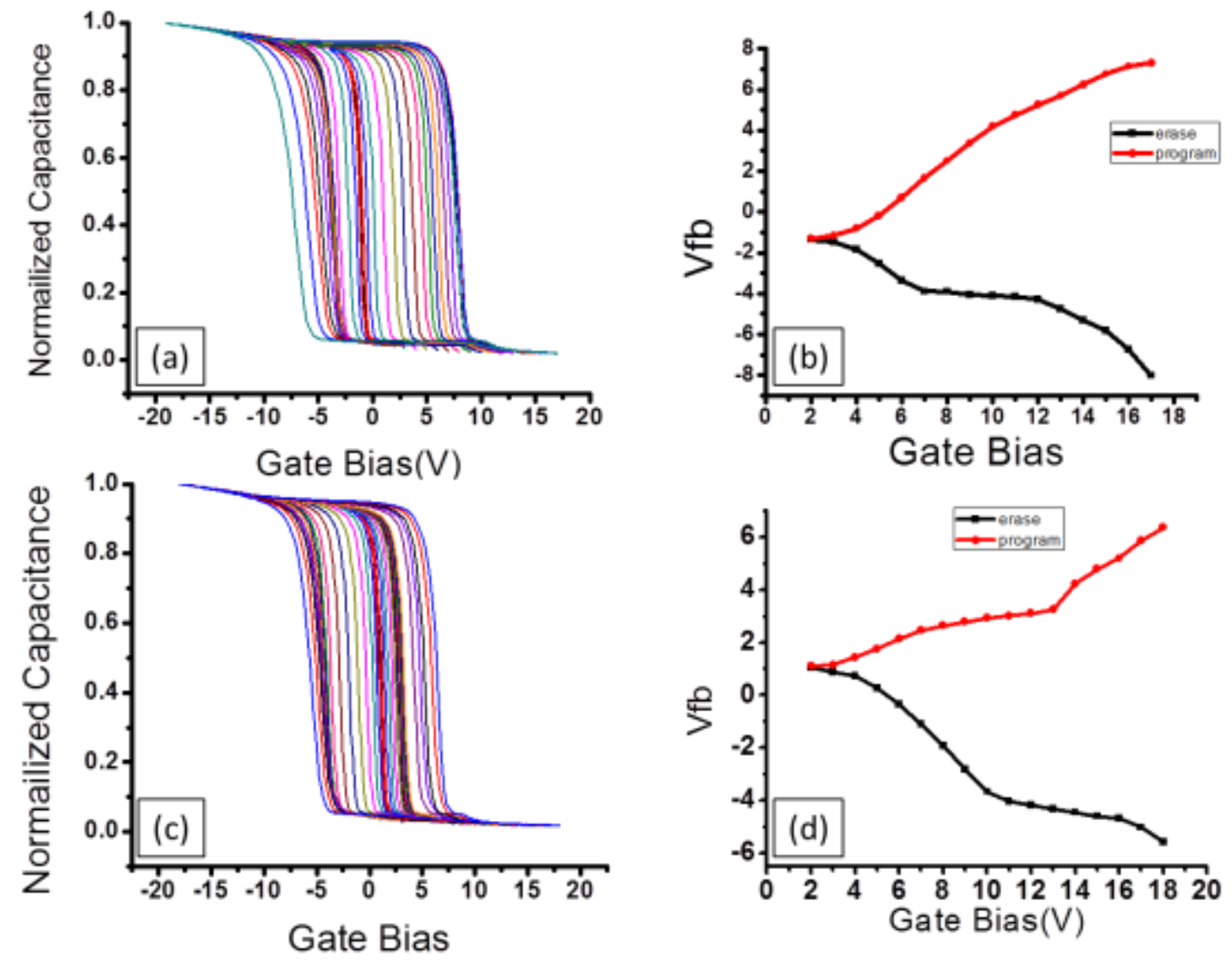

Figure 3-11: Shows the CV characteristics and erase programming efficiency for a NVM MOSCAP with (a-b)3nm Tunneling, 5 sec Pt NP(TA=23.8), 4nm Separation $\mathrm{Al}_{2} \mathrm{O}_{3}$ layer, 20 sec Pt NP(TA=23.8) and 15nm capping (c-d) 3nm Tunneling, 5 sec Pt NP(TA=23.8), 4nm Separation $\mathrm{Al}_{2} \mathrm{O}_{3}$ layer, 20 sec

Pt NP(TA=16.2) and 15nm capping 
Here, an inverse effect is observed where the $V_{F B}$ shifts to the right because of the small size Pt NP at the second layer. The small size Pt NP at second layer corresponds to a higher coulomb energy associated with it and thus traps the electron in the bulk oxide. These trapped electrons in the second layer exhibit coulomb repulsion making it difficult to charge the first Pt NP layer. Also, this trapped electron corresponds to a shift in Vfb exhibiting asymmetric charging. Though, charging becomes a challenge with smaller Pt NP in second layer, the erasing operations becomes feasible exhibiting first layer saturation at -8 to $-12 \mathrm{~V}$ and creating a step-wise effect

Although the configurations in Figure 3-9, Figure 3-10, Figure 3-11, do not necessarily exhibit the multi-bit modes (or controlled charging), they are essential to endorse the fact that it is important to precisely control the Pt NP configurations and the thickness of the tunneling and inter-particle dielectric to see controlled layer by layer charging of Pt NP with electrons.

\subsubsection{Avoiding lateral tunneling and Coulomb staircase}

Apart from the electron tunneling from the first layer to the second layer directly, there could be two other mechanisms leading to an increase in memory window. 1) Addition of extra electrons in the metal nanoparticle exhibiting coulomb blockade staircase

effect, and 2) Lateral tunneling of electrons within the same NP layer. In order to validate the claims in these experiments it is important to prove that these two mechanisms are not liable for the demonstrated effects. Firstly, For single layer Pt NP device, the electron can be continuously injected to the charge trapping Pt NP during the programming operation, however the flatband voltage can reach a saturation as long as the bias potential lower than 
the next level of Pt NP charging energy. For coulomb blockade staircase effect to take place the energy required at the $5 \mathrm{sec}$ Pt nanoparticle to add another electron from equation (ii) is calculated to be $1264 \mathrm{meV}$ (with $\mathrm{n}=2$ ). This high energy is clearly not provided through the $\varphi$ potential at the NP, thus this mechanism doesn't hold true for the configuration studied here. Moreover, as capacitance coupling depends on the number of trapped charges in the Pt NP, coulomb repulsion will not allow more than 1 electron per particle.

\subsection{References}

[1] ITRS. (2011. Available: http://www.itrs.net/reports.html

[2] H.-C. Chien, C.-H. Kao, J.-W. Chang, and T.-K. Tsai, "Two-bit SONOS type Flash using a band engineering in the nitride layer," Microelectronic Engineering, vol. 80, pp. 256-259, 2005.

[3] Y. J. Seo, K. C. Kim, H. D. Kim, M. S. Joo, H. M. An, and T. G. Kim, "Correlation between charge trap distribution and memory characteristics in metal/oxide/nitride/oxide/silicon devices with two different blocking oxides, <equation $><$ font $\quad$ face='verdana' $>$ Al $</$ font $\rangle\langle$ sub $>2</$ sub $><$ font face $=$ 'verdana' $>\mathrm{O}</$ font $>\langle$ sub $>3</$ sub $><$ /equation $>\quad$ and $\quad<$ equation $><$ font face='verdana' $>\mathrm{SiO}</$ font $><$ sub $>2</$ sub $></$ equation $>$," Applied Physics Letters, vol. 93, pp. 063508-063508-3, 2008.

[4] S. Suk-Kang, P. Il-Han, L. Chang Ju, L. Yong Kyu, J. D. Lee, B.-G. Park, C. Soo Doo, and K. Chung Woo, "Fabrication and program/erase characteristics of 30-nm SONOS nonvolatile memory devices," Nanotechnology, IEEE Transactions on, vol. 2, pp. 258-264, 2003. 
[5] H.-T. Lue, K.-Y. Hsieh, and C.-Y. Lu, "Overview of Advanced 3D Charge-trapping Flash Memory Devices," MRS Online Proceedings Library, vol. 1250, pp. null-null, 2010.

[6] S. Tiwari, F. Rana, H. Hanafi, A. Hartstein, E. F. Crabbe, and K. Chan, "A silicon nanocrystals based memory," Applied Physics Letters, vol. 68, pp. 1377-1379, 1996.

[7] H. I. Hanafi, S. Tiwari, and I. Khan, "Fast and long retention-time nano-crystal memory," Electron Devices, IEEE Transactions on, vol. 43, pp. 1553-1558, 1996.

[8] S. Tiwari, F. Rana, K. Chan, L. Shi, and H. Hanafi, "Single charge and confinement effects in nano-crystal memories," Applied Physics Letters, vol. 69, pp. 1232-1234, 1996.

[9] K. Ya-Chin, K. Tsu-Jae, and H. Chenming, "Charge-trap memory device fabricated by oxidation of $\mathrm{Si}<\mathrm{sub}>1-\mathrm{x} \quad</ \mathrm{sub}>\mathrm{Ge}<\mathrm{sub}>\mathrm{x}</$ sub $>$," Electron Devices, IEEE Transactions on, vol. 48, pp. 696-700, 2001.

[10] Z. Liu, C. Lee, V. Narayanan, G. Pei, and E. C. Kan, "Metal nanocrystal memories. I. Device design and fabrication," Electron Devices, IEEE Transactions on, vol. 49, pp. 1606-1613, 2002.

[11] Z. Liu, C. Lee, V. Narayanan, G. Pei, and E. C. Kan, "Metal nanocrystal memoriespart II: electrical characteristics," Electron Devices, IEEE Transactions on, vol. 49, pp. 1614-1622, 2002.

[12] J.-Y. Tseng, C.-W. Cheng, S.-Y. Wang, T.-B. Wu, K.-Y. Hsieh, and R. Liu, "Memory characteristics of Pt nanocrystals self-assembled from reduction of an embedded $\mathrm{PtO}\left[\mathrm{sub}_{\mathrm{x}}\right]$ ultrathin film in metal-oxide-semiconductor structures," Applied Physics Letters, vol. 85, pp. 2595-2597, 2004. 
[13] S. Tiwari, F. Rana, H. Hanafi, A. Hartstein, E. F. Crabbé, and K. Chan, "A silicon nanocrystals based memory," Applied Physics Letters, vol. 68, p. 1377, 1996.

[14] D. Zhao, Y. Zhu, R. Li, and J. Liu, "Simulation of a Ge-Si hetero-nanocrystal memory," Nanotechnology, IEEE Transactions on, vol. 5, pp. 37-41, 2006.

[15] S. Maikap, P. Tzeng, H. Lee, C. Wang, T. Tien, L. Lee, and M.-J. Tsai, "Physical and electrical characteristics of atomic layer deposited TiN nanocrystal memory capacitors," Applied Physics Letters, vol. 91, pp. 043114-043114-3, 2007.

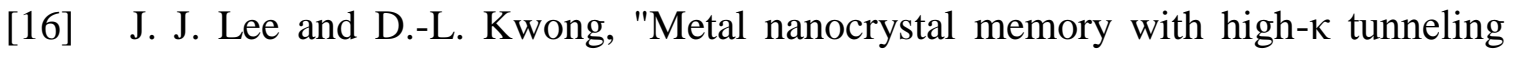
barrier for improved data retention," Electron Devices, IEEE Transactions on, vol. 52, pp. 507-511, 2005.

[17] H. Tsuji, N. Arai, T. Matsumoto, K. Ueno, Y. Gotoh, K. Adachi, H. Kotaki, and J. Ishikawa, "Silver nanoparticle formation in thin oxide layer on silicon by silver-negativeion implantation for Coulomb blockade at room temperature," Applied Surface Science, vol. 238, pp. 132-137, 2004.

[18] J. Welser, S. Tiwari, S. Rishton, K. Lee, and Y. Lee, "Room temperature operation of a quantum-dot flash memory," Electron Device Letters, IEEE, vol. 18, pp. 278-280, 1997.

[19] Y. Minseong, R. Balavinayagam, and G. Shubhra, "Room temperature observation of size dependent single electron tunneling in a sub-2 $\mathrm{nm}$ size tunable Pt nanoparticle embedded metal-oxide-semiconductor structure," Nanotechnology, vol. 22, p. 465201, 2011. 
[20] M. Yun, B. Ramalingam, and S. Gangopadhyay, "Multi-Layer Pt Nanoparticle Embedded High Density Non-Volatile Memory Devices," Journal of The Electrochemical Society, vol. 159, pp. H393-H399, 2012.

[21] Y. Minseong, D. W. Mueller, M. Hossain, V. Misra, and S. Gangopadhyay, "Sub2 nm Size-Tunable High-Density Pt Nanoparticle Embedded Nonvolatile Memory," Electron Device Letters, IEEE, vol. 30, pp. 1362-1364, 2009.

[22] J. R. C. Jeff, M. Yun, B. Ramalingam, B. Lee, V. Misra, G. Triplett, and S. Gangopadhyay, "Charge storage characteristics of ultra-small Pt nanoparticle embedded GaAs based non-volatile memory," Applied Physics Letters, vol. 99, pp. 072104-3, 2011.

[23] C.-C. Wang, Y.-K. Chiou, C.-H. Chang, J.-Y. Tseng, L.-J. Wu, C.-Y. Chen, and T.B. Wu, "Memory characteristics of $\mathrm{Au}$ nanocrystals embedded in metal-oxidesemiconductor structure by using atomic-layer-deposited Al 2 O 3 as control oxide," Journal of Physics D: Applied Physics, vol. 40, p. 1673, 2007.

[24] T. Z. Lu, M. Alexe, R. Scholz, V. Talelaev, and M. Zacharias, "Multilevel charge storage in silicon nanocrystal multilayers," Applied Physics Letters, vol. 87, p. 202110, 2005.

[25] F. D. K. a. G. S. M, " Transport in Nanostructures (Cambridge: Cambridge University Press)," 1997.

[26] M. Yun, B. Ramalingam, and S. Gangopadhyay, "Room temperature observation of size dependent single electron tunneling in a sub-2 $\mathrm{nm}$ size tunable Pt nanoparticle 
embedded metal-oxide-semiconductor structure," Nanotechnology, vol. 22, p. 465201, 2011.

[27] J. Yeargain and C. Kuo, "A high density floating-gate EEPROM cell," in Electron Devices Meeting, 1981 International, 1981, pp. 24-27.

[28] D. C. Guterman, I. H. Rimawi, T.-L. Chiu, R. D. Halvorson, and D. McElroy, "An electrically alterable nonvolatile memory cell using a floating-gate structure," Electron Devices, IEEE Transactions on, vol. 26, pp. 576-586, 1979.

[29] R. Shiner, J. Caywood, and B. Euzent, "Data retention in EPROMs," in Reliability Physics Symposium, 1980. 18th Annual, 1980, pp. 238-243.

[30] N. R. Mielke, "New EPROM data-loss mechanisms," in Reliability Physics Symposium, 1983. 21st Annual, 1983, pp. 106-113.

[31] R. L. Puurunen, "Surface chemistry of atomic layer deposition: A case study for the trimethylaluminum/water process," Journal of Applied Physics, vol. 97, p. 121301, 2005.

[32] B. Ramalingam, S. Mukherjee, C. J. Mathai, K. Gangopadhyay, and S. Gangopadhyay, "Sub-2 nm size and density tunable platinum nanoparticles using room temperature tilted-target sputtering," Nanotechnology, vol. 24, p. 205602, 2013.

[33] J. Herrmann, D. J. Bray, K. H. Müller, G. Wei, and L. F. Lindoy, "Tuning the Coulomb charging energy in cross-linked nanoparticle films," Physical Review B, vol. 76, p. $212201,2007$. 
[34] J. Dufourcq, S. Bodnar, G. Gay, D. Lafond, P. Mur, G. Molas, J. P. Nieto, L. Vandroux, L. Jodin, F. Gustavo, and T. Baron, "High density platinum nanocrystals for non-volatile memory applications," Applied Physics Letters, vol. 92, pp. 073102-3, 2008.

[35] A. Corporation, "Maxwell Student Version," 3.1.04 ed. Pittsburgh, PA: Ansoft Corporation, 2002.

[36] Wasshuber, C., About single-electron devices and circuits1998: Österr. Kunst-u. Kulturverl 


\section{CHAPTER 4: Neutron Detectors with integrated Pt NP and ${ }^{10} \mathrm{~B}$}

\subsection{INTRODUCTION}

Neutron detectors have applications in many fields as area monitors and personal dosimeters in health physics and homeland security applications. They are also used in biological applications-> to measure absorbed ionization radiation in human tissue for radiation, -> to measure neutron, gamma and beta exposure to monitor radiation levels in space and nuclear powered vessels, and $\rightarrow$ in commercial nuclear power plants for monitoring spent atomic fuel and in-core and out- core instrumentation. They also play an important role for detection of nuclear-based Weapons of Mass Destruction (WMD) using special nuclear materials (SNMs) in cargo containers at port of entries. SNM typically emits neutrons making the detection of these materials feasible. Due to the extremely important nature of neutron related applications, and their hazardous consequences to health and safety, it is important to detect neutrons using efficient systems. Neutron detectors are mainly classified as non-powered and powered. Although, non-powered detectors like thermo-luminescent[1], bubble[2] and track-etch[3] dosimeters have an advantage due to their cost and simplicity, they cannot be used for instantaneous real time neutron detection and thus one choses powered detectors for real time detection. The most commonly used powered neutron detector is a $\mathrm{He}^{3}$ proportional counter, where a gas filled tube is exposed to the ionizing particle of interest [4]. This leads to a gas ionization process and a current due to an avalanche process which is determined through the ionization energy of the incident particle. Since the ionization of gas molecules is dependent on the 
incident neutron, the kinetic energy of the neutron can be determined by estimating the number of ionized gas molecules in the chamber [4]. These detectors are often accompanied by bulky enclosures and high power batteries to power up the electronics and analyzers involved. This is where portable solid-state-device (SSD) based detectors come into play.

Any detection process requires a measureable change of energy when the detector encounters the detecting agent. This change in energy depicts a detectors efficiency to detect - for example, how fast it happens and the magnitude of the change with respect to the detecting-agent interaction. For traditional neutron interaction with materials, this change in energy is typically based on properties like ionization potential [4], but these changes are not sensitive and selective enough to be of practical use as real-time neutron detection schemes. Reliable energy transduction technology relies upon radiation-induced effects in the electrical structure of a device to produce a plausible signal $[5,6]$, however, the ionizing nature of neutrons significantly limits the probability of such reactions occurring locally, which are often undetected. For simple SSD based detectors, neutron reactions with inorganic semiconductors such as $\mathrm{Si}$ and GaAs do not have high enough cross sections to produce sensitive thermal neutron detectors [7] It is for this reason that two material isotopes of interest in neutron detection studies are ${ }^{6} \mathrm{Li}$ and ${ }^{10} \mathrm{~B}$ :which are capable of undergoing specialized capture reactions with low-energy neutrons that can convert the difficult-to-detect neutral particle (neutron) into energetic charged-particle. ${ }^{6} \mathrm{Li}$ has a capture cross-section of 940 Barns compared to 3840 Barns for ${ }^{10} \mathrm{~B}$, due to which typically ${ }^{10} \mathrm{~B}$ based compounds are preferred for neutron capture converter materials[8]. 
Thermal neutrons absorbed by ${ }^{10} \mathrm{~B}$ produce ${ }^{7} \mathrm{Li}(1.015 \mathrm{MeV})+{ }^{4} \mathrm{He}(1.777 \mathrm{MeV})$ with a branching ratio of $94 \%$ and ${ }^{7} \mathrm{Li}(0.84 \mathrm{MeV})+{ }^{4} \mathrm{He}(1.470 \mathrm{MeV})+0.482 \mathrm{MeV}$ gamma ray with a branching ratio of $6 \%$. The ${ }^{7} \mathrm{Li}$ and ${ }^{4} \mathrm{He}$ are charged particles with a traversing range of $2.7-3.0 \mu \mathrm{m}$ and $5.1-6.4 \mu \mathrm{m}$ in decaborane $\left(\mathrm{B}_{10} \mathrm{H}_{14}\right)$ respectively[7]. For solid state detectors, to make neutron detection feasible, a converter (or active sensing) layer comprised of ${ }^{10} \mathrm{~B}$ or ${ }^{6} \mathrm{Li}$ is usually incorporated within the active vicinity of the operating PN junction or MOSFET device to achieve desirable changes in the operating behavior upon neutron interaction and charged particle interaction [8-12]. A detection event occurs when a decelerating charged particle upon neutron capture creates electronhole pairs in (or close to) the converter region of the radiation sensor.

Figure 4-1shows the reaction mechanism for neutron capture for ${ }^{10} \mathrm{~B}$. As depicted in the figure, the energetic particles are produced at a phase difference of 180 degree to each other and these energetic particles create e-h pairs that contribute to sensing mechanisms. Typically, all solid state neutron detectors generate and separate these electron-hole pair through a hetero-structure geometry. The direct-conversion resistive or dielectric hetero-structures are consequently the most efficient architectures, which are often limited by lack of materials in which the majority constituent is a high-neutroncapture-cross-section isotope that also yields energetic particles upon neutron capture and is capable of generating and separating $\mathrm{e}-\mathrm{h}$ pairs. 

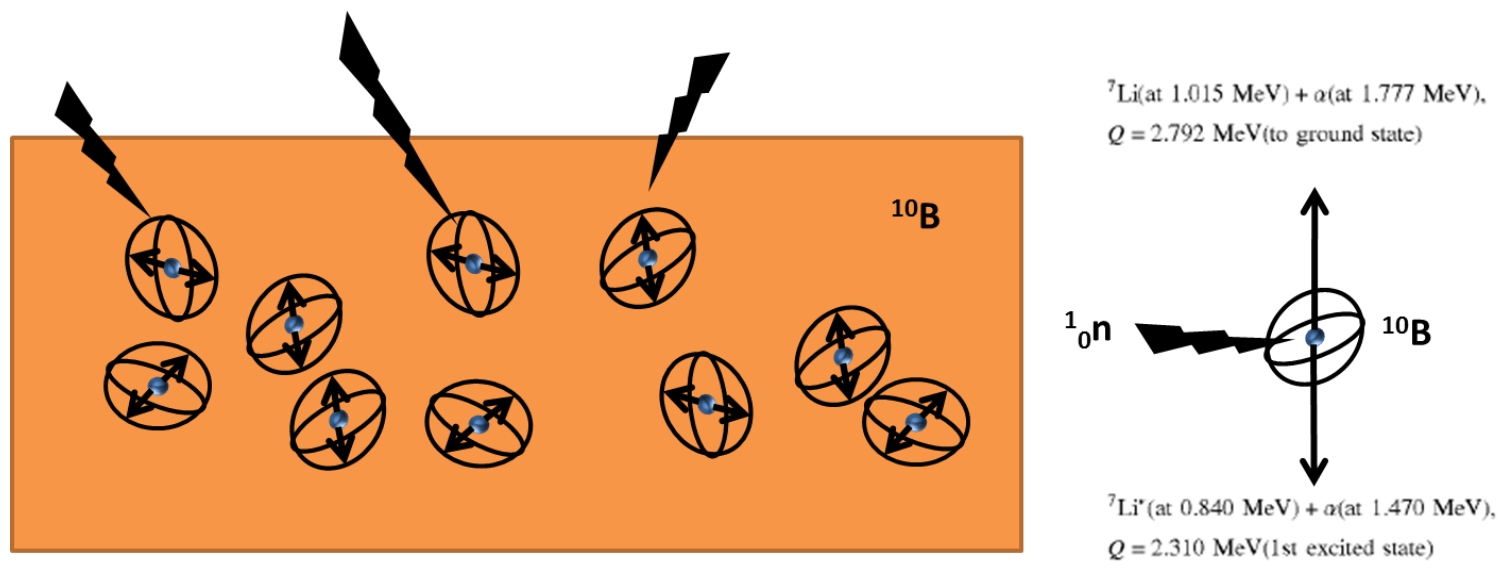

$$
{ }^{10} B+{ }_{0}^{1} n \rightarrow\left\{\begin{array}{cc}
6 \%: & { }^{7} \mathrm{Li}(\text { at } 1.015 \mathrm{MeV})+\alpha(\text { at } 1.777 \mathrm{MeV}), \\
& Q=2.792 \mathrm{MeV}(\text { to ground state }) \\
94 \%: & { }^{7} \mathrm{Li}^{*}(\text { at } 0.840 \mathrm{MeV})+\alpha(\text { at } 1.470 \mathrm{MeV}), \\
Q=2.310 \mathrm{MeV}(1 \text { st excited state })
\end{array}\right.
$$

Figure 4-1 (a) Interpretation of neutron interaction in a Boron enriched converter layer (b) reaction products in 180 degree phase 
This limitation for direct conversion hetero-structures has driven most of the recent research towards studying and optimizing PN junction and Schottky devices[8, 12]. Especially, these architectures are based on an indirect conversion where the neutron conversion material is placed in the vicinity of the device, so that the scattering or reaction capture products may create e-h pairs in the space charge region for a noticeable change in current characteristics. Due to the highly directional nature ( $180^{\circ}$ phase shifted) of neutroncapture reaction products, only a fraction of them take part in signal transduction limiting even the theoretical efficiency of detection $<10 \%$. Experimentally, only a maximum of 3.7\% detection efficiency has been observed. Currently, research led by McGregor et al. focuses on development of perforated and thin film coated semiconductor thermal neutron detectors, mostly as PIN diodes[10-12]. They use such converter materials to convert neutron capture into energetic components detectable through their PIN diode configuration. The presence of a depletion region in these devices allows them to operate as a neutron detector. The charge carriers created by the passing energetic particles are carried out of the depletion region by the electric field to create a current pulse proportional to the number of created charges. The sensitivity of these devices are increased by operating them in reverse bias with a larger depletion region, but their efficiency are still limited by the probability of energetic particles arriving at the junction (due to the directionality of reaction products). To improve the energetic particle interaction with their PN junctions, Mc Gregor et al etched trenches in their substrate to backfill it with ${ }^{10} \mathrm{~B}$ based neutron converter materials and used the neutron capture event to efficiently collect the energetic particle for a detection signal[10-12]. This technique, though still based on an indirect architecture, results in an intrinsic efficiency of 30\% for neutron detection, and thus 
is still limited by the capturing efficiency of the traversing energetics particles which are highly directional.

SRAM (Static Random Access Memory) detectors have also surfaced in recent years where the converter layer materials are back filled in a Silicon-On-Insulator (SOI)based SRAM cell. In these structures, upon neutron exposure, a soft error involving a change of state is caused which instantaneously changes the digital information in the memory cell [13-15]. For all these configurations, the converter layer is used as an indirect conversion layer where it is not a part of the device. Thus, these suffer from low detection efficiency due to an indirect architecture and irreversible radiation damage[16], making them unreliable and expensive $[8,9,11,12]$.

In this chapter, we would discuss development of a highly sensitive neutron detector with ${ }^{10} \mathrm{~B}$ enriched Pt Nanoparticles (Pt NP) embedded MOSCAP structures, where the neutron converter layer acts as a dielectric- making it an active part of the device, thereby defining it as a direct conversion architecture[8]. We explore new methods to separate and sustain charges in direct-conversion dielectric hetero-structures, which has been and continues to be a grand challenge. We address this challenge by developing a novel method to incorporate metal nanoparticles (NP) as charge trap centers embedded in the dielectric layer of the MOSCAP architectures. The NP and ${ }^{10} \mathrm{~B}$ based material serves towards both neutron capture (by ${ }^{10} \mathrm{~B}$ ) as well as hold generated charges (by Pt NP) for usable signal transduction. The ${ }^{7} \mathrm{Li}$ and ${ }^{4} \mathrm{He}$ are charged particles with a range of $2.7-3.0$ $\mu \mathrm{m}$ and $5.1-6.4 \mu \mathrm{m}$ in carborane, respectively. A detection event occurs when a decelerating charged particle generated upon neutron capture creates electron-hole pairs in or at the interface to the converter region of the device. Most importantly, the embedded 
Pt nanoparticles act as trap centers for any charge separation generated by primary reaction products of a neutron capture event. Similar analogies were observed in various applications of these NPs through several years[17]. Using these NP, we have seen phenomenal charge trapping and retention for MOSCAP devices due to the Coulomb blockade effect [18-21]. The unique properties of these NPs were utilized in highly sensitive and fast detection of explosive vapors through efficient charge transfer between $\mathrm{NP}$ and explosive molecule [22]. In another application, $\mathrm{TiO}_{2}$ nanowires decorated with $\mathrm{Pt}$ NPs exhibited extremely high $\mathrm{CO}_{2}$ photoreduction efficiency due to the fast electrontransfer rate in $\mathrm{TiO}_{2}$ single crystals and the efficient electron-hole separation by the Pt NPs [23]. In this work, we exploit these unique charge separation properties to capture charges generated through energetic reaction product interaction leading to detection of neutrons.

\subsection{Experimental details:}

\subsubsection{Device Fabrication}

For the different type of capping oxides (PS/DB and $\mathrm{Al}_{2} \mathrm{O}_{3}$ ), control samples were fabricated with no embedded Pt NP layers. Dual layer devices (similar to one in CHAPTER 3:) and control sample were fabricated with a polystyrene and decaborane mixture as capping oxides. Traditional $\mathrm{Si}$ and $\mathrm{Al}_{2} \mathrm{O}_{3}$ based MOSCAP with embedded Pt NP were also fabricated to compare and study detection schemes. The samples were prepared on modified Shiraki[24]-cleaned P-type Silicon wafers with 5-10 $\Omega-1-\mathrm{cm}-1$ resistivity terminated with a $0.9 \mathrm{~nm}$ chemical oxide. Tunneling $\mathrm{Al}_{2} \mathrm{O}_{3}$ dielectric layers were deposited by atomic layer deposition (ALD, Cambridge Nanotech) at $350{ }^{\circ} \mathrm{C}$ using water and Trimethylaluminum (TMA) precursors with a deposition rate of $0.8 \AA$ Acycle. The TMA 
precursors for the ALD system were purchased from Sigma-Aldrich. Since hydroxylterminated surfaces are essential for complete TMA reaction coverage and defect-free $\mathrm{Al}_{2} \mathrm{O} 3$ formation[25], water was pulsed for twenty cycles $(0.02 \mathrm{~s}$ pulses with $8 \mathrm{~s}$ purge interval) prior to flowing any TMA vapor. This was followed by 35 repetitive cycles of $0.015 \mathrm{~s}$ TMA and $0.02 \mathrm{~s} \mathrm{H}_{2} \mathrm{O}$ with $8 \mathrm{~s}$ purge interval. The 35 cycles lead to a total of $3.22 \mathrm{~nm}$ of tunneling oxide (thickness measured using ellipsometer a J.A. Woollam ellipsometer) 


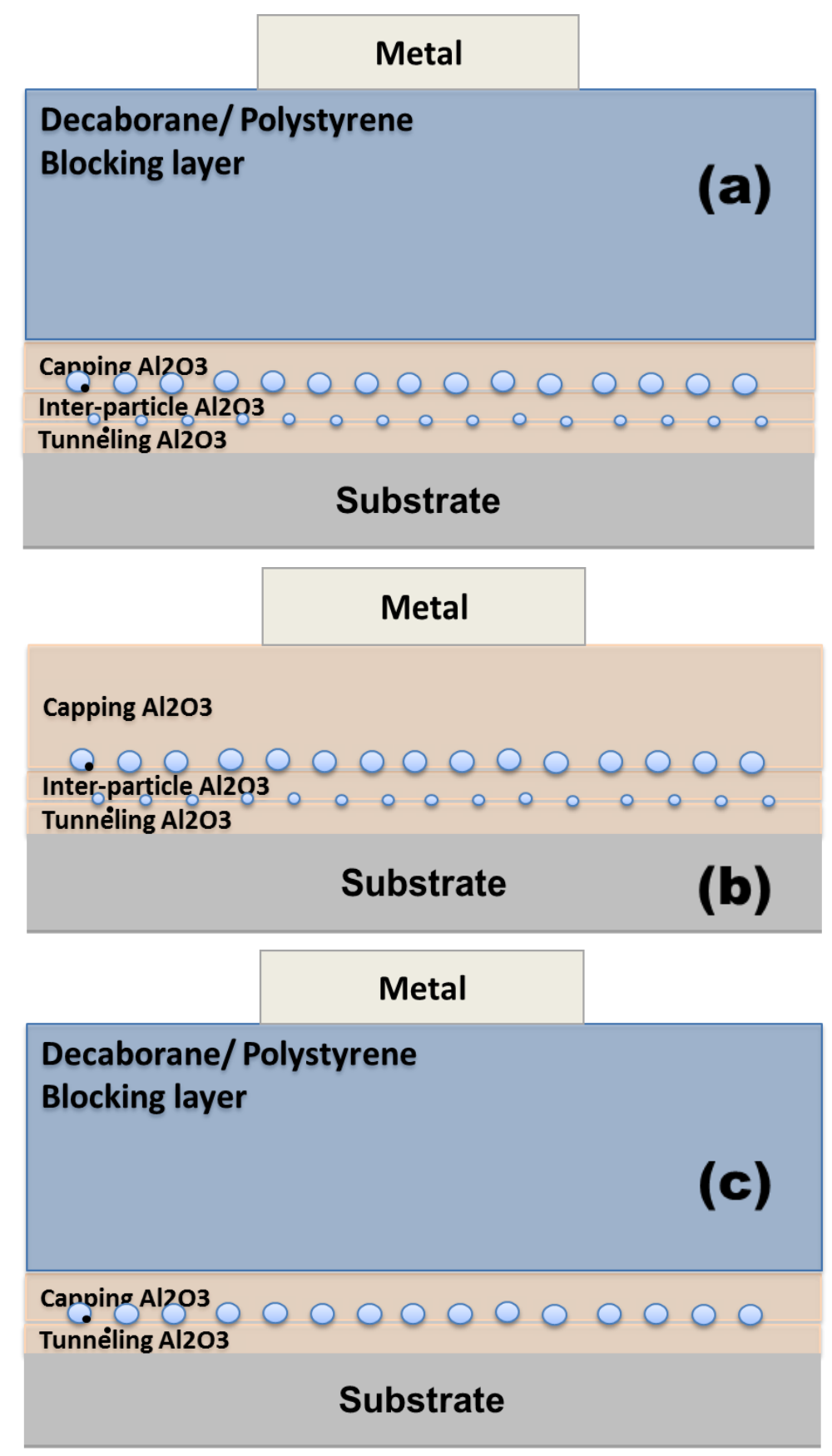

Figure 4-2: Device Schematic for Si based Pt NP embedded MOSCAP device (a)Dual layer Pt NP with ${ }^{10} B$ enriched Decaborane/Polystyrene capping layer (b) Dual Layer Pt NP embedded MOSCAP without Polystyrene/Decaborane layer, and (c)Single Layer Pt NP embedded MOSCAP with Polystyrene/Decaborane capping layer 
Table 4-1: Different Layers with thickness and method of deposition
Layer
Thickness/size
Method

\begin{tabular}{|l|l|l|}
\hline Bare Si substrate with & $0.9 \mathrm{~nm}$ & Chemical - Shiraki \\
\hline $\mathbf{S i O} 2$ & $3.2 \mathrm{~nm}$ & ALD \\
\hline Tunneling Al2O3 & $0.5 \mathrm{~nm}$ & Tilted Target Sputtering \\
\hline Pt NP & $3.2 \mathrm{~nm}$ & ALD \\
\hline Inter dielectric & $1.11 \mathrm{~nm}$ & Tilted Target Sputtering \\
\hline Pt NP & $3.2 \mathrm{~nm}$ & ALD \\
\hline Blocking Al2O3 & $122 \mathrm{~nm}$ & Spin coating \\
\hline $\begin{array}{l}\text { Capping } \\
\text { Polystyrene/Decaborane }\end{array}$ & & \\
\hline
\end{tabular}


After the tunneling layer deposition, for devices depicted in Figure 4-2 (a) and (b) the first Platinum nanoparticle (Pt NP) layer was deposited using a tilted target configuration with 23.8 degree target angle, $30 \mathrm{~W}$ deposition power and $5 \mathrm{~s}$ deposition time as detailed previously[17]. For dual layer devices (Fig 2(a-b)) the first Pt NP layer was followed by depositing a $3 \mathrm{~nm}$ inter particle $\mathrm{Al}_{2} \mathrm{O}_{3}$ layer at $100^{\circ} \mathrm{C}$, followed by the second $\mathrm{Pt} \mathrm{NP}$ layer deposited at 23.8/20s/30W. For capping oxides, two variations were made for a controlled experiment on understanding the interaction:

For traditional $\mathrm{Si} / \mathrm{Al}_{2} \mathrm{O}_{3}$ memory devices (Figure 4-2 (b)), where the devices were capped with a $15 \mathrm{~nm} \mathrm{Al}_{2} \mathrm{O}_{3}$ using $\mathrm{ALD}$, followed by $150 \mathrm{~nm}$ Ti electrode deposition. For the neutron detector MOSCAP structure, the devices were capped with an interfacial $3 \mathrm{~nm}$ ALD-deposited $\mathrm{Al}_{2} \mathrm{O}_{3}$ and spin coated with Polystyrene $\left(\left(\mathrm{C}_{8} \mathrm{H}_{8}\right)_{\mathrm{n}}\right) /$ Decaborane $\left(\mathrm{B}_{10} \mathrm{H}_{14}\right)$ to deposit the neutron converter region. Capping oxide for devices in Figure 4-2(a),(c).This was followed by 100nm Ti and 50nm Au electrode deposition using a Kurt J. Lesker Axxis electron beam evaporator equipped with a multi-pocket deposition system. For devices shown in Figure 4-2 (c), single layer Pt NP were deposited using $23.8^{\circ} / 20 \mathrm{~s}$ and $23.8^{\circ} / 45 \mathrm{~s}$ atop the $3 \mathrm{~nm}$ tunneling $\mathrm{Al}_{2} \mathrm{O}_{3}$ layer. These were followed by $3 \mathrm{~nm} \mathrm{Al}_{2} \mathrm{O}_{3}$ and $122 \mathrm{~nm}$ PS/DB layer and Ti/Au electrode deposition.

${ }^{10}$ B Neutron Converter Layer synthesis: $90 \mathrm{mg}$ Polystyrene and $250 \mathrm{mg}$ Decaborane was mixed in Toluene to form a Polystyrene-Decaborane mixture. This was then spin-coated on Si based memory stack to form the converter film. These films were then cured in vacuum for 12 hours (room temperature) for slow polymerization and the heated at 90C and 150C for 10 minutes in a Nitrogen filled glove box to remove any intrinsic solvent. The ellipsometry measured thickness of this film was $122.227 \mathrm{~nm}$. 


\subsubsection{Neutron Exposure}

Both the device types- traditional and Ps-DB based, were exposed to a wellcharacterized mixed field thermal neutron beam in the University of Missouri - Research Reactor (MURR). The gamma dose measured at the irradiation position is $64 \mathrm{cGy} / \mathrm{h}$ and the thermal neutron flux is $8.5 \times 10^{8} \mathrm{n} / \mathrm{cm}^{2} / \mathrm{s}$ at $0.023 \mathrm{eV}$. Figure $4-3$ shows the neutron source distribution plot for the beam created at MURR, the samples were strategically placed such that only the thermal component of the beam was used for irradiation, in this case the $0.0253 \mathrm{eV}$ peak was used. For detector response and analysis, these devices were exposed for different durations to control the number of incident neutrons. Au fission foils estimated

the different doses to be 1.19E10, 1.615E10, 3.0175E10 and 5.1E10 $\mathrm{n} / \mathrm{cm}^{2}$. Samples without any exposure were also retained for comparison and control. 


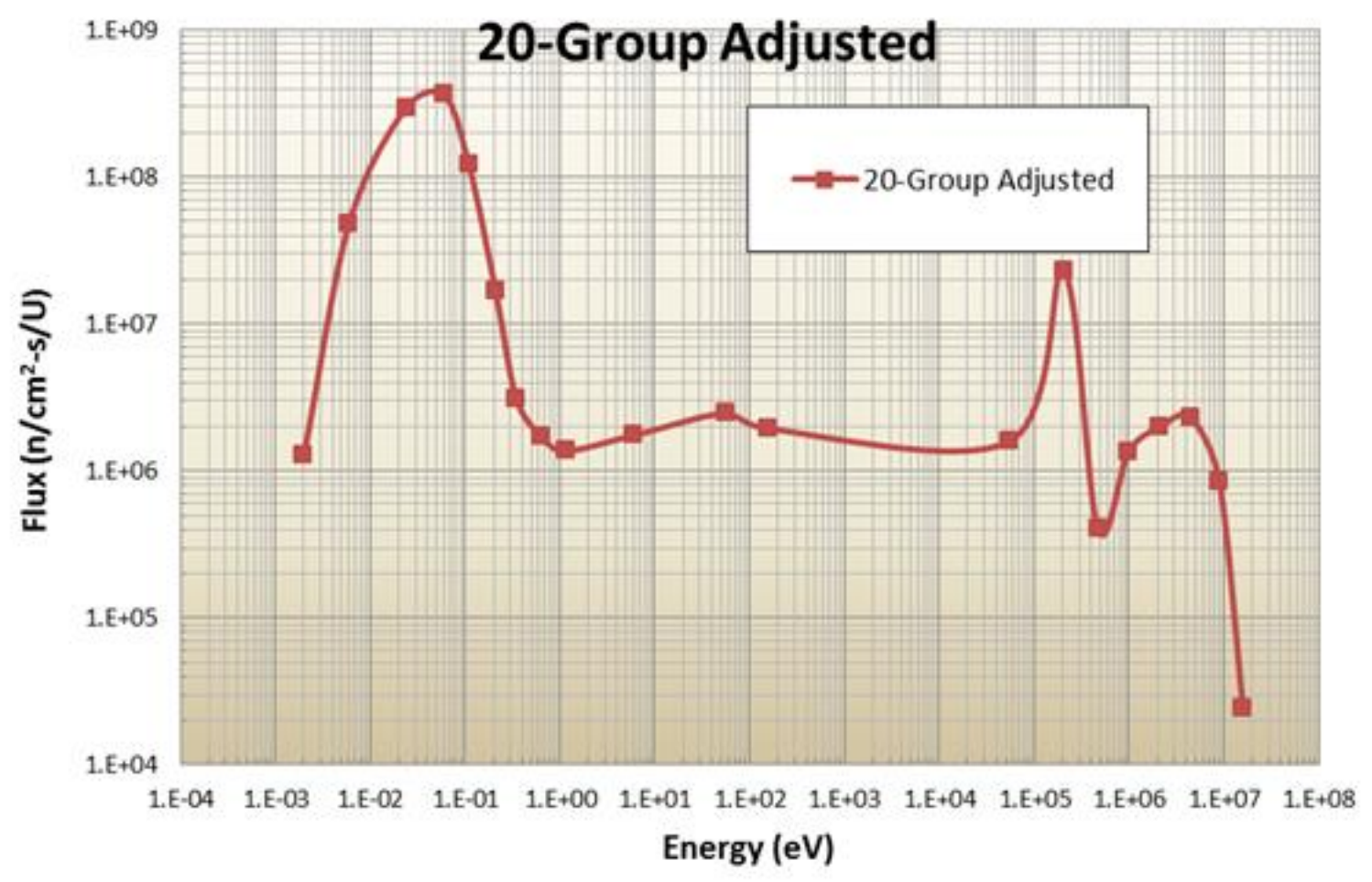

Figure 4-3: Unshielded neutron source distribution plot 


\subsubsection{Electrical characterization}

After neutron exposure, the devices were immediately measured for observable changes in their behavior. Capacitance-Voltage $(\mathrm{C}-\mathrm{V})$ measurements were performed at room temperature in a 4200SCS Keithley semiconductor characterization instrument. A shielded probe station from Signatone micromanipulators was used as a testing station.

\subsection{Results and discussion}

For proper understanding and a systemic study, the devices shown in Fig 5-2 are discussed in separate sections to emphasize the importance of their individual attributes towards device configuration.

\subsubsection{Neutron exposure on Samples with PS/DB capping layer and no embedded Pt NP:}

Figure 4-6 shows the capacitance voltage curves for the device with $122 \mathrm{~nm}$ Decaborane/ Polystyrene layer and no embedded Pt NP. Plots 4-4 (a) through (e) shows the $\mathrm{C}-\mathrm{V}$ plot at zero neutron exposure, 1.19E10, 1.615E10, 3.0175E10 and 5.1E10 neutrons $/ \mathrm{cm}^{2}$ respectively. Fig $4-4(f)$ shows the compiled plot for comparing the $\mathrm{C}-\mathrm{V}$ curves where it is evident that there is no change in the response for these devices. These devices were fabricated to analyze the importance and the role of Pt NP in the sensor system.

Though these devices didn't show a characteristic neutron response, it was important to study and understand the behavior of the newly synthesized dielectric material composed of $\mathrm{B}_{10} \mathrm{H}_{14}$ (Decaborane) using $\mathrm{C}-\mathrm{V}$. Conventionally, parameters derived from the $\mathrm{C}-\mathrm{V}$ analysis can classify the type of dielectric and identify the necessary properties 
pertaining to dielectric behavior. Especially, any irregularity in an ideal C-V curve for traditional Si-based MOSCAP (like flat band shift, stretched C-V, hysteresis etc.), will directly relate to its properties and its interface with the semiconductor[26]. For the PS/DB devices studied here, the ideal flat band for these devices with the metal-semiconductor work-function difference is at -0.35 , but the experimental flat-band is at $-2.7 \mathrm{~V}$ for most devices in Figure 4-6. This negative shift in flat band voltage for control sample denotes trapped charges in the dielectric which is purely contributed through the electron deficient PS/DB layer. According to Cotton's Advanced Inorganic Chemistry (pg 188, $5^{\text {th }}$ edition, 1988); boranes are electron deficient because there are not enough electrons to allow formation of conventional 2 electron bonds between all adjacent pairs of boron atoms[27]. This leads to covalent bonds that are not observed in carbon based alkane systems. For example, there are 3 center 2 electron B-B-B bonds and 2 center 2 electron bonds $\mathrm{B}-\mathrm{B}, \mathrm{B}-\mathrm{H}$ and $\mathrm{B}-\mathrm{H}_{2}$, leading to incredibly complex chemistry. In many of the boranes, the boron atoms are capable of acting as electron pair acceptors in an attempt to complete the octet. In the case of $\mathrm{B}_{10} \mathrm{H}_{14}$ there are 14 electron vacancies in a lewis structure to satisfy the octet rule. As such, boranes are often lewis acids which are electron acceptors. Since the dielectric is enriched with these acceptor atoms we see a negative shift in the Voltage flat band. Currently, for these devices a negative shift of -2.35 is observed corresponding to $1.9 \times 10^{11}$ holes $/ \mathrm{cm}^{2}$ as trapped charge density in the oxide.

For neutron detection, a measurable detection for MOSCAP can be through a shift in $\mathrm{C}-\mathrm{V}$ curve after neutron exposure, which will correspond to the charge trapping ability of a device. With trapped charges, the flat-band voltage of the device shifts such that the direction and the magnitude of this shift $\left(\Delta \mathrm{V}_{\mathrm{FB}}\right)$ can be used to predict the number of 
charges using the relationship $N_{t}=\left(C_{o x} \Delta \mathrm{V}_{\mathrm{FB}}\right) / q$, where $N_{t}$ is the trapped charge, $C_{o x}$ is the accumulation capacitance density of the oxide, and $q$ the elementary charge. For these devices in Figure 4-6, we believe that the neutron converter region captures and converts the incident neutron to energetic reactions products of mostly ${ }^{7} \mathrm{Li}(1.015 \mathrm{MeV})+{ }^{4} \mathrm{He}$ $(1.777 \mathrm{MeV})$, where these energetic products create electron-hole pairs through their traversing path. Though generated, the e-h pairs are not trapped due to which they get recombined and lost, making it impossible to convert the transduction to a usable signal. This becomes a traditional example of direct-dielectric architecture which is capable of generating electron-hole pairs but fails to separate them through this architecture, which is a major limitation for devices of such type. But by itself, these devices showed optimum device performance using a polymer dielectric enriched with ${ }^{10} \mathrm{~B}$ (decaborane). Based on present literature, this is the first investigation of such devices in MOSCAP configurations ever reported. From the $\mathrm{C}-\mathrm{V}$ curves, for a $\mathrm{C}_{\mathrm{ox}}$ of $1.3 \mathrm{e}-8 \mathrm{~F} / \mathrm{cm}^{2}$ and a dielectric thickness of 122.227 $\mathrm{nm}$ PS-DB and $9.4 \mathrm{~nm} \mathrm{Al}_{2} \mathrm{O}_{3}$, the experimental dielectric constant is calculated to be 1.93 , which is close to the reported dielectric constant of pure Polystyrene films (2.6) [28]. This reduction in overall dielectric constant could be due to the addition of decaborane in polystyrene. 


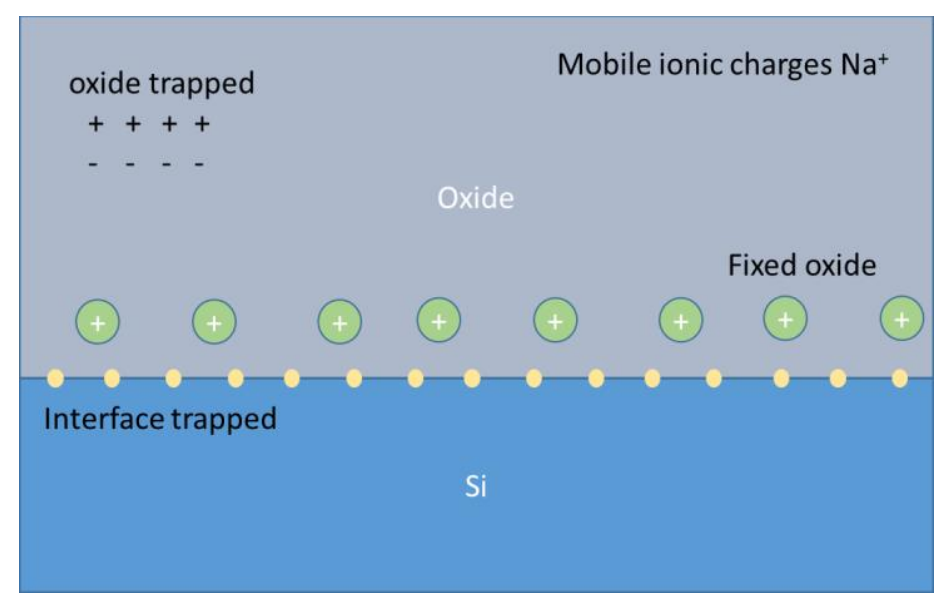

Figure 4-4: Different types of oxide charges

In a typical MOSCAP, a voltage shift in flat band is due to the sum of all charges

$$
\Delta \mathrm{V}_{\mathrm{fb}}=\frac{Q_{f}+Q_{m}+Q_{o t}}{C_{o x}}
$$

Where the $\mathrm{Q}_{\mathrm{f}}$ is the fixed oxide charge, $\mathrm{Q}_{\mathrm{m}}$ is the mobile ionic charge, and $\mathrm{Q}_{\mathrm{t}}$ is the trapped oxide charge.

For a MOSCAP, the flat band is given by

$$
\mathrm{V}_{\mathrm{fb}}=\Phi_{\mathrm{ms}}-\frac{Q_{f}+Q_{m}+Q_{o t}}{C_{o x}}
$$

Where $\Phi_{\mathrm{ms}}$ is the work function difference. The oxide charges are specific to their type and location and effectively change $\mathrm{C}-\mathrm{V}$ characteristics. As shown in Fig, $\mathrm{Q}_{\mathrm{f}}$ is located at the interface of the $\mathrm{Si}_{-} \mathrm{Al}_{2} \mathrm{O}_{3}$, it is generally positive and its density is not greatly affected by the oxide thickness or by the type or concentration of impurities in the silicon, but it depends on oxidation and annealing conditions, and on silicon surface orientation. Mobile ionic charges are throughout the oxide and can move back and forth through the oxide 
layer, depending on biasing conditions, and thus give rise to voltage shifts. The oxide trapped charge is associated with defects in the oxide. The oxide traps are usually initially neutral and are charged by introducing electrons and holes into the oxide layer. This can occur from any current passing through the oxide layer, hot-carrier injection, or by photon excitation.

Hysteresis can be due to interface states or at higher biases due to mobile ions and trapped charges. For thinner films, the E-field generated due to the applied bias is typically high enough to move these mobile charges or to charge the trap locations within the dielectric at low bias conditions. However, for thicker films the hysteresis is primarily due to interface states at low bias conditions and often require high biasing to move the mobile charges and activate the trap sites in the thick dielectric. In the case of control sample without any PS/DB layer, with a $\sim 20 \mathrm{~nm} \mathrm{Al}_{2} \mathrm{O}_{3}$ we see hysteresis at low bias due to interface states and some mobile/trapped charges $(0.15 \mathrm{~V}$ at $\pm 1 \mathrm{~V})$. At higher biases, the effect of mobile charges on hysteresis is more pronounced leading to a hysteresis of around $1.3 \mathrm{~V}$ at a sweep of $\pm 8 \mathrm{~V}$. In case of control devices with PS/DB layer, the hysteresis remains consistent at around $0.1 \mathrm{~V}$ (less than $0.15 \mathrm{~V}$ as seen in the control case without PS/DB) even at higher biasing operation up to $\pm 8 \mathrm{~V}$, which is most likely due to just the interface states where mobile and trapped charges are not contributing. The absence of larger hysteresis at higher bias operation indicates that we are not moving mobile charges or activating trapped charges (which would contribute to hysteresis) within the operating bias regime. This is because the dielectric layer $122 \mathrm{~nm} P S / D B+6 n m \mathrm{Al}_{2} \mathrm{O}_{3}$ is too thick to generate a high enough E-field to move the mobile charges (or activate trap sites) through the dielectric. 

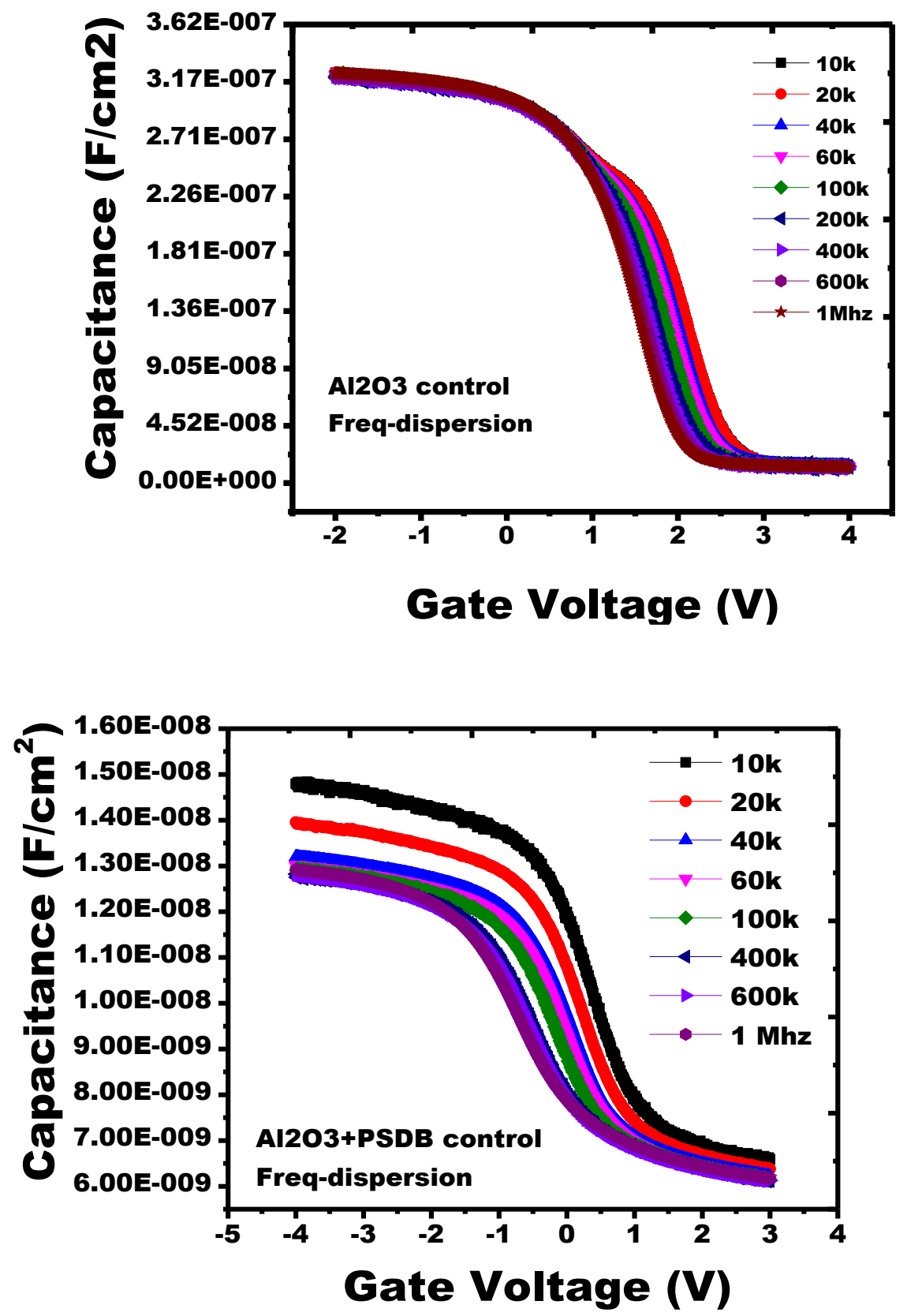

Figure 4-5: Frequency Dispersion C-V plot for (a) $20 \mathrm{~nm}$ Al2O3 Control sample without PS/DB capping and (b) 6nm Al2O3 with 122nm PSDB capping oxide MOSCAP. 
Since for the thick PS/DB layer, the contribution from the mobile charges and trapped charges are minimal for the studied biasing conditions, thus it is logical to conclude that the $-2.35 \mathrm{~V}$ flat band shift is primarily due to the fixed charges. It should be noted that fixed charges are primarily due to the processing condition of the dielectric. These fixed charges were not observed in control sample without PS/DB which leads us to the conclusion that the processing condition (like annealing at $90 \mathrm{C}$ and $150 \mathrm{C}$ for PS/DB film) of the dielectric contribute towards creating the fixed charges near the Si-Alumina interface and results in a flat band shift. It is well known that polymer dielectrics have lot of mobile ions which during annealing are likely to penetrate the Alumina layer and result in fixed charges near the interface. From Figure 4-5, when we compare PS/DB (a) and control Alumina without PS/DB (b), it can be seen that the PS/DB C-V curves are stretched and show a high degree of frequency dispersion compared to the Alumina control, which is generally attributed to charging near the interface. Based on the evidence so far it is probable that this results in change in $\mathrm{V}_{\mathrm{fb}}$. 

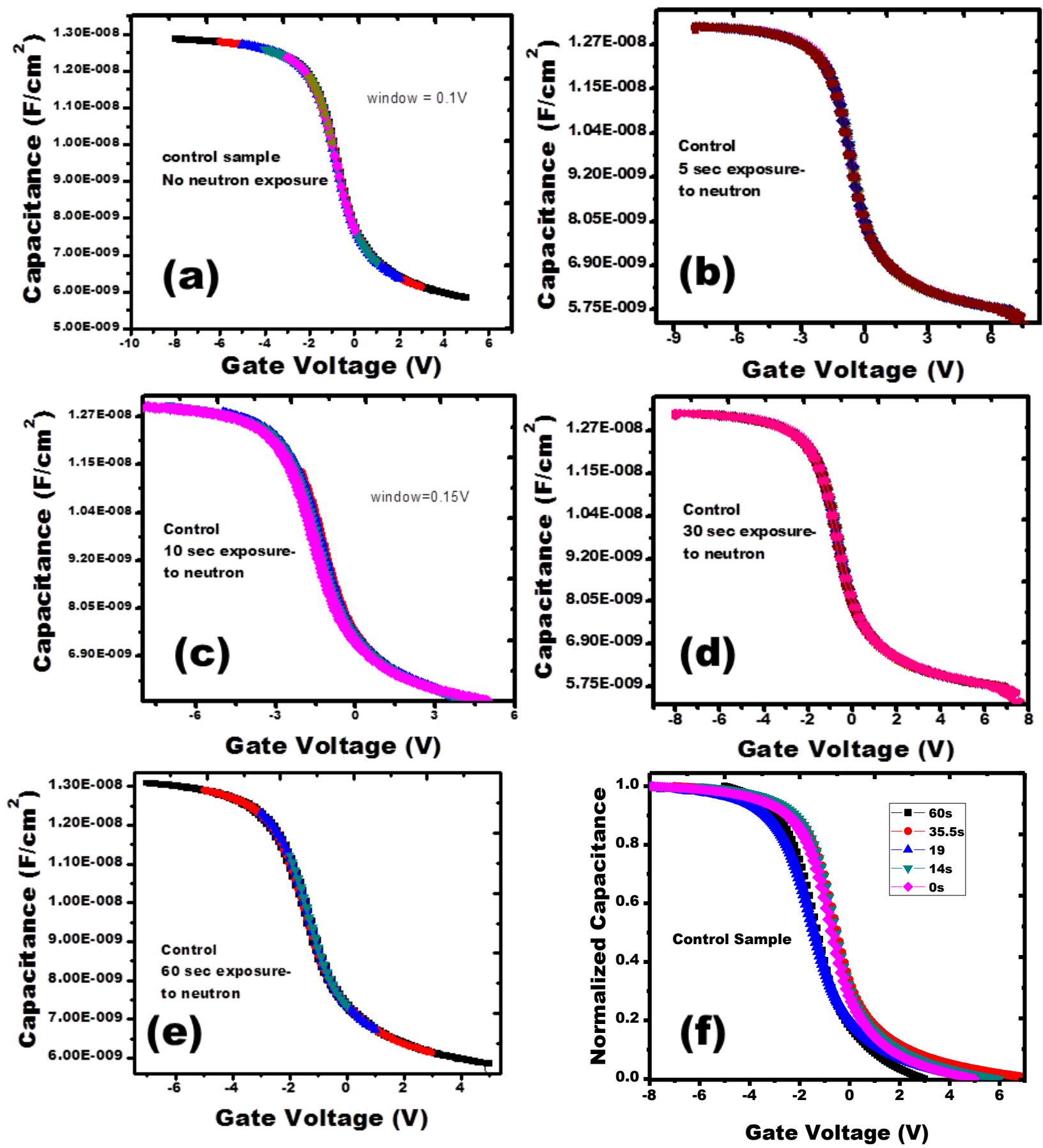

Figure 4-6:C-V plots for Control sample with no embedded Pt NP with stack configurations of Si/9 $\mathrm{nm} \mathrm{Al} \mathrm{O}_{3} / 122 \mathrm{~nm} \mathrm{PS} / \mathrm{DB}$ layer; exposed to neutron dose of (a) $0 \mathrm{n} / \mathrm{cm}^{2}$ (b) $1.19 \mathrm{E} 10 \mathrm{n} / \mathrm{cm}^{2}$, (c)1.615E10 $\mathrm{n} / \mathrm{cm}^{2},(\mathrm{~d}) 3.0175 \mathrm{E} 10 \mathrm{n} / \mathrm{cm}^{2}$ and (e) $5.1 \mathrm{E} 10 \mathrm{n} / \mathrm{cm}^{2}$.(f) shows the combination of all plots for comparison. 


\subsubsection{Samples with PS/DB capping layer and embedded Single layer Pt NP:}

Figure 3-2 shows the TEM images for Pt NP deposited by tilted target configuration at tilt angle/deposition times of $23.8^{\circ} / 5 \mathrm{~s}(0.52 \pm 0.12 \mathrm{~nm}), 23.8^{\circ} / 20 \mathrm{~s}(1.11 \pm 0.28 \mathrm{~nm})$ and $23.8^{\circ} / 45 \mathrm{~s}(1.45 \pm 0.36 \mathrm{~nm})$. This configuration allows tunable size and density in the sub-nm range described elsewhere [17]. This unique ability to control the nanoparticle size and density in the sub-nm regime has been the basis of controlled charging and effective retention. With coulomb blockade energy ranging from $100 \mathrm{meV}$ up to $300 \mathrm{meV}$, there has been evidence of high density charging and phenomenal retention with devices using embedded Pt NP in their dielectric[17, 22, 29, 30]. Table 4-2 shows the Coulomb charging energy of specific sized Pt NPs with $3 \mathrm{~nm}$ tunneling $\mathrm{Al}_{2} \mathrm{O}_{3}$ layer.

Table 4-2 Pt NP size and its associated Coulomb charging energy

\begin{tabular}{lll}
\hline $\begin{array}{l}\text { Deposition } \\
\text { time }\end{array}$ & $\begin{array}{l}\text { Pt Nanoparticle } \\
\text { diameter }(\mathbf{n m})\end{array}$ & $\begin{array}{l}\text { Coulomb charging } \\
\text { energy }(\mathbf{m e V})\end{array}$ \\
\hline $\mathbf{5}$ & 0.54 & 336 \\
$\mathbf{2 0}$ & 1.11 & 147 \\
$\mathbf{4 5}$ & 1.45 & 62.8 \\
\hline
\end{tabular}

As described in $[29,30]$, the charging of Pt nanoparticle is highly dependent on the Coulomb charging energy and the Quantum confinement energy. Higher energy means that the ability to discharge a Pt NP (remove an electron) will need a higher matching potential energy. For Single layer Pt NP embedded in $\mathrm{Al}_{2} \mathrm{O}_{3}$ dielectric of $3 \mathrm{~nm}$ tunneling and $15 \mathrm{~nm}$ 
capping oxide, we can derive the biasing voltage required to program and erase the device. Typically for stacks with Si/Alumina/Pt NP/Alumina/ Ti there will be capacitance coupled from both the tunneling layer and the blocking layer. The sum of these capacitances will give us the total capacitance induced at the NP and thus the required energy.

For a spherical NP with Sphere-plane configuration

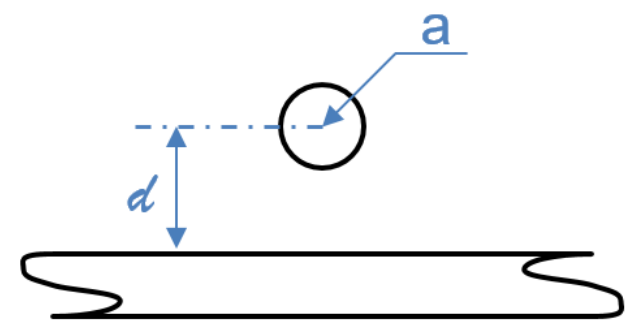

The capacitance is given by [31]

$$
C=2 \pi \epsilon \sqrt{d^{2}-4 a^{2}} \sum_{j=0}^{\infty}\left(\operatorname{coth}\left[\left(j+\frac{1}{2}\right) \operatorname{arcosh}\left(\frac{d}{2 a}\right)\right]-1\right)
$$

Or

$$
C=4 \pi \varepsilon_{\text {medium }} a\left[1+\frac{a}{2 d}+\frac{\left(\frac{a}{2 d}\right)^{2}}{1-\left(\frac{a}{2 d}\right)^{2}}\right]
$$


Where ' $a$ ' is the radius of the nanoparticle and ' $d$ ' is the distance from the surface of the electrode to the center of the nanoparticle. Using these, equations for $\mathrm{C}_{\mathrm{BO}}$ (blocking) and $\mathrm{C}_{\mathrm{TO}}$ (tunneling) can be computed

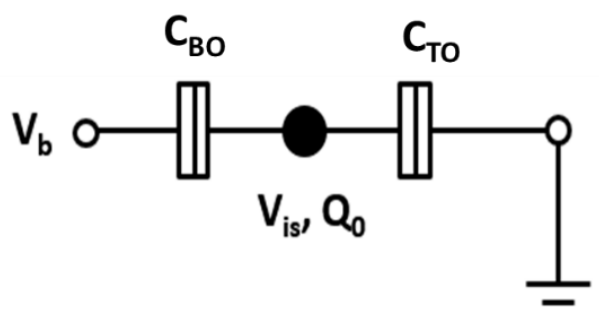

Total capacitance associated to the NP is

$$
C_{t o t}=C_{T O}+C_{B O}
$$

It must be noted that even though it looks like the capacitors are in series in the figure, for the actual capacitance coupled at the nanoparticle, they will act as parallel capacitors and thus it will be a summation of ingredient capacitances.

Table 4-3 Capacitance terms associated to the $0.5 \mathrm{~nm}$ Pt NP

$\begin{array}{lrrr} & \text { NP size } & \text { oxide thickness } & \text { Capacitance } \\ \text { Сто } & 0.5 & 3.2 & 2.3092 \mathrm{E}-19 \\ \text { Сво } & 0.5 & 15 & 2.24402 \mathrm{E}-19 \\ \text { Стот } & & & 4.55322 \mathrm{E}-19\end{array}$


Table 4-3 shows the self-capacitance calculated for $0.5 \mathrm{~nm}$ Pt NP. To derive the voltage required for charging one electron onto a nanoparticle, the work done should be 'equal to'/'greater than' change in the energy at the NP.

$$
U_{N}(\mathrm{~N})=\frac{e^{2}\left(N-\frac{1}{2}\right)}{C_{\text {tot }}}=\mathrm{W}=\mathrm{e} V_{b} C_{B O} / C_{t o t}
$$

Where $\mathrm{N}$ is the number of electrons, $\mathrm{V}_{\mathrm{b}}$ is the bias voltage.

If we include quantum confinement, the total energy required for addition of electron will be quantum confinement energy (Q.C.E) + Coulomb charging energy. Thus, the work done should be matched by applied $\mathrm{V}_{\mathrm{b}}$, and the equation becomes,

$$
U_{N}(\mathrm{~N})=\mathrm{Q} . \mathrm{C} . \mathrm{E}+\frac{e^{2}\left(N-\frac{1}{2}\right)}{C_{\mathrm{tot}}}=\mathrm{W}=\mathrm{e} V_{b} C_{B O} / C_{t o t}
$$

Where Q.C. E for different sized Pt NP (Table 4-3) can be calculated using [31], (Ef for Pt is $5.6 \mathrm{eV}$ )

Energy level Spacing $\frac{d E f}{d N}=\frac{2}{3} \frac{E f}{N}$

$\mathrm{E} f=$ Fermi Energy of the bulk material

$\mathrm{N}=$ number of atoms in the nanoparticle [17]

Table 4-4: Quantum confinement energy for different Pt NP

$\begin{array}{lr}\text { NP size } & \text { QCE(meV) } \\ 0.5 & 663.3333 \\ 0.7 & 234.1177 \\ 1.11 & 57.68116 \\ 0.65 & 284.2857\end{array}$


Adding Coulomb charging energy to Q.C.E and calculating $V_{b}$, for the case of 0.5 $\mathrm{nm}$ Pt NP in a regular $3 \mathrm{~nm}$ tunneling and $15 \mathrm{~nm}$ capping $\mathrm{Al}_{2} \mathrm{O}_{3}$ stack, we get a required voltage of $1.7024 \mathrm{~V}$ and for $1.11 \mathrm{~nm}$ Pt NP we get a required voltage of $0.275 \mathrm{~V}$. This voltage delivers the required energy through a created E-Field around the NP which accelerates the programming/erasing behavior. In the case of PS/DB samples, the problem becomes a more complex phenomena since the capacitance coupled to the blocking oxide is through two different oxide materials (the capping layer is $3 \mathrm{~nm} \mathrm{Al}_{2} \mathrm{O}_{3}$ and $122 \mathrm{~nm}$ PS/DB). Thus, to simplify, we calculate the E-Field required for programming and erasing in an $\mathrm{Al}_{2} \mathrm{O}_{3}$ system. This E-field around the $\mathrm{Pt} \mathrm{NP}$ is matched in the PS/DB devices in order to calculate the required biasing for program/erase operation. For example, in the $18 \mathrm{~nm}$ $\mathrm{Al}_{2} \mathrm{O}_{3}$ samples without PS/DB layer, the programming voltage for $1.11 \mathrm{~nm}$ Pt NP was calculated to be $0.27 \mathrm{~V}$ creating a total E-field of $15 \mathrm{MV} / \mathrm{m}$. In order to derive the programming voltage of similar systems but with a $122 \mathrm{~nm}$ PS/DB capping layer the Efield was matched to $15 \mathrm{MV} / \mathrm{m}$ in the layer around Pt NP and the required gate voltage was calculated using the voltage divider rule for the different dielectric material. A $4.4 \mathrm{~V}$ of equivalent gate voltage was calculated to create a $15 \mathrm{MV} / \mathrm{m}$ E-field around the Pt NP in devices with $122 \mathrm{~nm}$ PS/DB layer. Similarly for the $0.5 \mathrm{~nm} \mathrm{Pt} \mathrm{NP}$, the $1.7 \mathrm{~V}$ requirement corresponds to $36.09 \mathrm{~V}$ gate voltage requirement in a PS/DB capping layer device, and for $1.4 \mathrm{~nm} \mathrm{Pt} \mathrm{NP}$ the requirement is calculated to be $2.4 \mathrm{~V}$. Thus, for single layer devices embedded with Pt NP the direct relation of NP size and the voltage required to program becomes a key governing factor to determine the usability of these devices as neutron detectors. 
Table 4-5: Required Gate voltage to exhibit programming in devices with 122nm PS/DB capping layer

\begin{tabular}{l|l} 
NP Size & Required Gate Voltage \\
\hline $1.4 \mathrm{~nm}$ & $2.4 \mathrm{~V}$ \\
$1.11 \mathrm{~nm}$ & $4.4 \mathrm{~V}$ \\
$0.5 \mathrm{~nm}$ & $36.09 \mathrm{~V}$
\end{tabular}

Table 4-5 becomes very important to determine the type of NP that can be used for usable signal transduction. When a neutron capture event is converted to energetic particle, the e-h pair generation in different device layers are created and trapped in these NPs energetically. Since this study is based on post exposure measurements, when the devices are swept with gate voltages to measure the $\mathrm{C}-\mathrm{V}$ curves, the devices with $1.11 \mathrm{~nm}$ and $1.4 \mathrm{~nm}$ becomes unusable since the sweeping voltage will typically remove the collected charges after a neutron detection event. Thus, only devices with $0.5 \mathrm{~nm}$ Pt NP as their first layer in a dual layer achitecture becomes applicable for neutron detection using post exposure measurement techniques, where the energetically programmed Pt NP retains its charges even during a sweep voltage of $\pm 10 \mathrm{~V}$.

Fig 4-5 shows the C-V (hysteresis) curves for devices with embedded $1.11 \mathrm{~nm}$ and $1.4 \mathrm{~nm}$ Pt NP, were there was no memory window for $1.11 \mathrm{~nm}$ Pt NP but a slight window (4.36V) for 1.4nm Pt NP was observed (Fig 4-5 (b)). Since these devices exhibited memory windows, their ability to program and erase the Pt NP by using the gate potential renders its use as neutron detectors for post-exposure measurements. This is mainly because, the trapped charges upon a neutron detection and energetic particle interaction will get driven out or erased during a read/write cycle making it impossible to determine a capture event. 
These devices were exposed to different neutron doses to verify their detection ability. Figure 4-7(a) shows the C-V curves for $1.11 \mathrm{~nm}$ Pt NP embedded devices under different neutron exposure where there was no observable response. We believe that upon neutron exposure when the traversing energetic particles are creating e-h pairs, the Pt NP might be trapping the electrons but losing it sooner due to retention ability of these Pt NPs. Since the charge is lost immediately after exposure, the trapping effect is not detectable for postexposure measurements. Similarly, for Figure 4-7 (b), the C-V curves showed a slight change in memory window which is again not quantifiable for reliable signal transduction. Moreover, as discussed, the electrical read/write ability is not desired for a reliable detection event. Though these devices failed to respond for post-exposure characterization, they will be excellent candidates for in-situ monitoring using MOSFET architectures, where a trapping event can correspond to shift in threshold voltage, thereby generating a pulse. The ability to remove the charges from Pt NP electrically, becomes beneficial at that point making the device re-usable. This has been demonstrated in a different scenario where MOSFET configurations were used for chemical analyte detection [22]. 

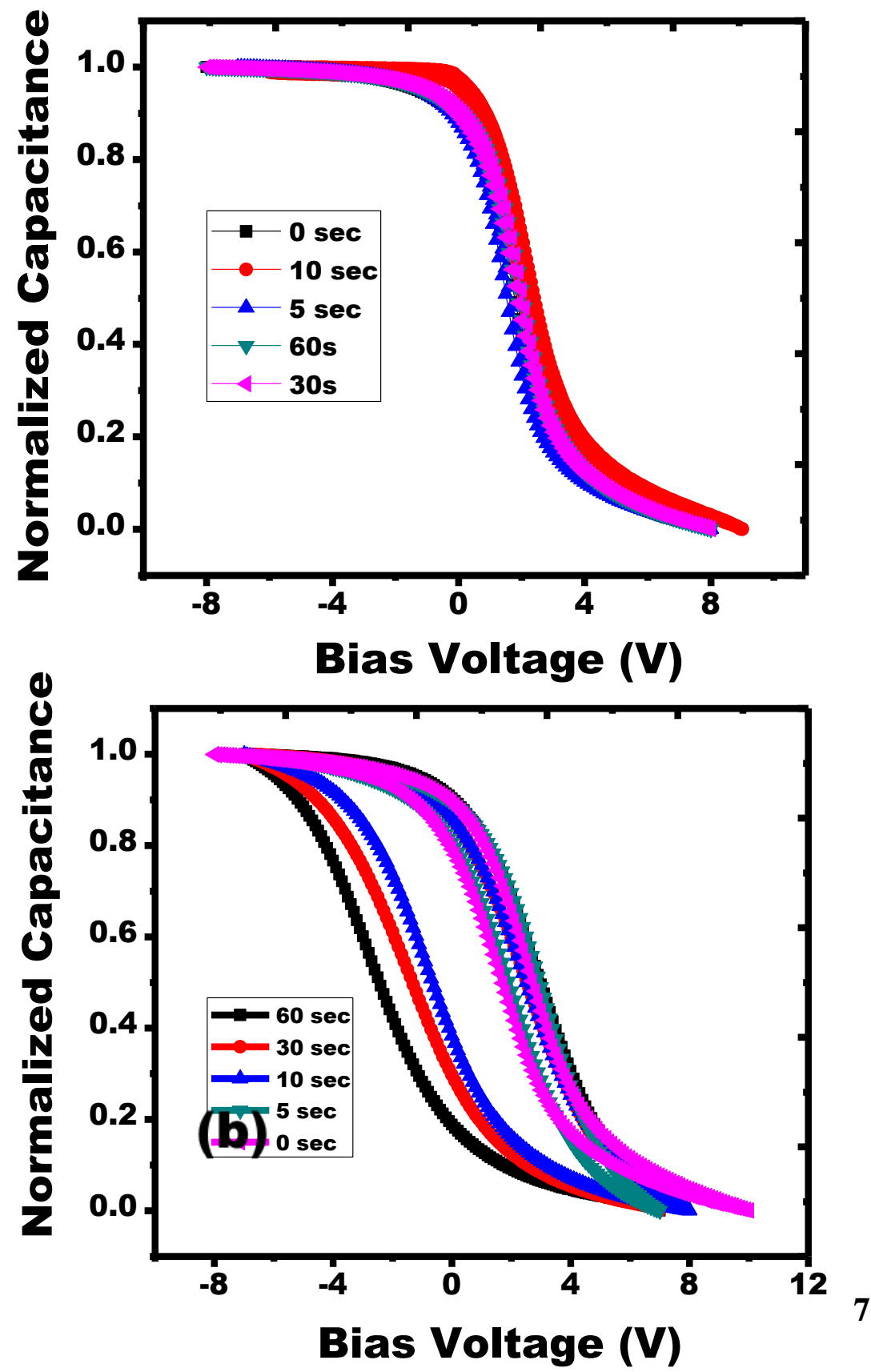

Figure 4-7: C-V plots for neutron exposure results for single layer Pt NP embedded MOSCAP with PS/DB layer embedded with (a)1.11 nm Pt NP and (b)1.45 nm Pt 


\subsubsection{Neutron exposure on Samples with PS/DB capping layer and embedded dual layer Pt NP:}

Pt NP with sizes $\sim 0.5 \mathrm{~nm}$ exhibit high coulomb blockade and charging energy which requires higher bias voltage to charge these NP, which falls out of the operation voltage $(\sim 35 \mathrm{~V}$ refer to section on page 141$)$. But once they are combined in a dual layer configuration with a larger particle, they get charged and would show exceptional retention ability[18, 30]. For such properties, these $0.5 \mathrm{~nm}$ Pt NP has been mainly used as a part of a dual layer system, and the role of the $0.5 \mathrm{~nm}$ Pt NP is to block any charge flow between the second layer and the Si/oxide interface (at low voltage ranges) and thus improving charge retention. For this work, the $0.52 \pm 0.12 \mathrm{~nm}$ and $1.11 \pm 0.28 \mathrm{~nm}$ PtNPs were chosen for their phenomenal charge retention properties when fabricated with PS/DB based dual layer devices (reported in chapter 4). The $0.52 \mathrm{~nm}$ Pt nanoparticle provides two advantages upon using them as the first layer: 1. it improves the retention and helps reduce any charge leakage to the semiconductor and 2. it increases the required voltage for programming $(35 \mathrm{~V}$ for devices with PS/DB layer). This advantage makes these devices instrumental for neutron detection studies. A second layer of $1.11 \mathrm{~nm}$ Pt NP was used for high density charge trap centers for efficient charge collection upon e-h pair generation during a neutron conversion event.

Figure 4-8 shows the C-V curves for dual layer Pt NP and 122nm Decaborane/Polystyrene capping layer as depicted in Fig 4-2(a). Figure 4-7(a) and 4-7(b) are two iterations of similar device stack but with different annealing temperatures(150C and 90C) of the PS/DB layer. The Figure 4-8 (a) denotes C-V for devices annealed at 150C and Figure 4-8 (b) denotes the $\mathrm{C}-\mathrm{V}$ curves for 90C annealing. As it can be noted, the 
devices in Figure 4-8 (a) are shifted to a flat band of $+0.5 \mathrm{~V}$ even at zero exposure. The film in these devices showed irregularity with topological cracks when annealed at 150C, which was adjudged to have degraded the device quality. This degradation has led to an unintentional charging of the embedded Pt NP which resulted in a positive shift of the flatband. For Fig Figure 4-8 (b), the devices annealed at 90C and zero neutron exposure, showed a flat bad shift of $\sim-2.6 \mathrm{~V}$, which is close to that observed in control samples, and therefore we believe that these devices had better performance compared to the one on Figure 4-8 (a). For both of these devices, due to a $3.2 \mathrm{~nm} \mathrm{Al}_{2} \mathrm{O}_{3}$ tunneling and a thick capping PS/DB dielectric, the required voltage to drive the charges in and out of the nanoparticle drastically increases making it impractical to charge the devices electrically. This observation leads to the design of a detection scheme, where energetic particles create electron-hole pairs in the vicinity of the NP, which gets trapped in the NP, changing the electrical characteristics of the device. Figure 4-8 shows the C-V plot at different neutron exposures ranging from zero exposure, 1.19E10, 1.615E10, 3.0175E10 and $5.1 \mathrm{E} 10 \mathrm{n} / \mathrm{cm}^{2}$ for both devices. . It is clearly evident there is a continuous shift in the voltage flat band as we increase the neutron dose corresponding to trapped charges in the dielectric. Upon neutron capture, the energetic reaction products generate eh pairs at the PS-DB layer, the $\mathrm{Al}_{2} \mathrm{O}_{3}$ layer and the $\mathrm{Si} / \mathrm{Al}_{2} \mathrm{O}_{3}$ interface, which are then trapped by the Pt NP, corresponding to a detection event. The high coulomb blockade effect and low self-capacitance property of the embedded ultra-small Pt NPs trap and retain the charges efficiently. This device, which is based on a direct-dielectric or insulator architecture, successfully generates and traps electrons, creating a measureable change for a detector response (representation shown in Figure 4-10). The absence of this effect in the $\mathrm{C}-\mathrm{V}$ of control sample and single 
layer samples (Fig 4-6) shows that the metal NP size and the way it is configured plays an important role in trapping these generated e-h pairs for neutron capture events, mainly for post-exposure measurements.
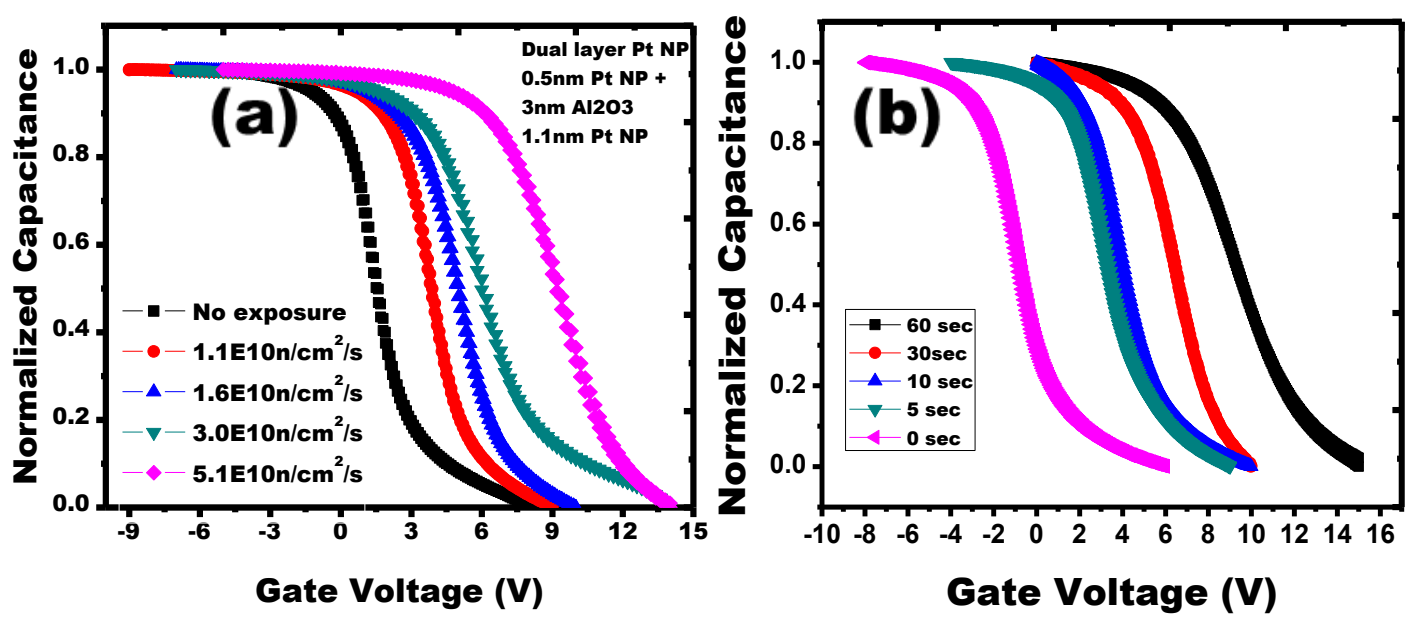

Figure 4-8: C-V plots for Dual layer Pt NP sample exposed to neutron dose of (a) 0 n/cm $\mathbf{c m}^{2}(\mathrm{~b})$ $1.19 \mathrm{E} 10 \mathrm{n} / \mathrm{cm}^{2},(\mathrm{c}) 1.615 \mathrm{E} 10 \mathrm{n} / \mathrm{cm}^{2},(\mathrm{~d}) 3.0175 \mathrm{E} 10 \mathrm{n} / \mathrm{cm}^{2}$ and $(\mathrm{e}) 5.1 \mathrm{E} 10 \mathrm{n} / \mathrm{cm}^{2}$.(f) shows the combination of all plots for comparison.

Figure 4-9 shows the detector response for $V_{F B}$ shift in the control sample and dual layer sample for both types of devices. Exposure 1 denotes the samples annealed at 150C with a compromised device performance and exposure 2 denotes the samples annealed at 90C with optimum device performance. In both cases it interesting to note that, the change in $\mathrm{V}_{\mathrm{FB}}$ for control sample in both exposures is within the error bar, but the change in $\mathrm{V}_{\mathrm{FB}}$ for Dual layer sample (from Figure 4-9 (f)) increases linearly with neutron exposure. More importantly, though the dual layer devices (for exposure 2) showed a large slope for the first exposure, we believe that these devices get saturated with charges produces due to the 
high neutron dose and we are limiting the detection. It is possible that with a lower neutron dose these device can exhibit a larger slope demonstrating phenomenal sensor response.

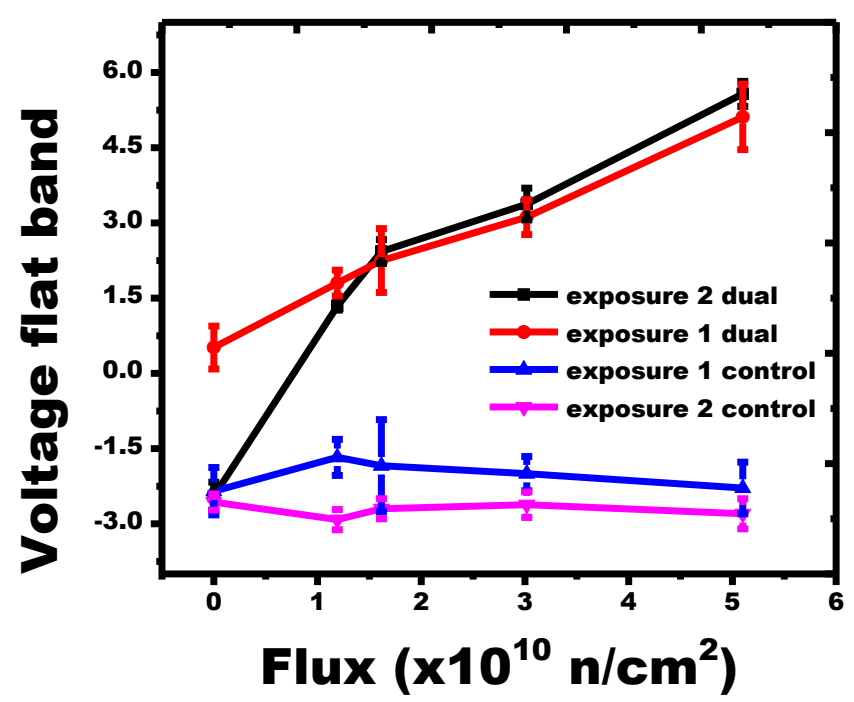

Figure 4-9: $V_{\mathrm{FB}}$ shift comparison for dual layer vs Control sample for different neutron exposure.

Furthermore, frequency dispersion measurements were carried out to show that these charging effects are purely from the bulk insulator and not from any $\mathrm{Si} / \mathrm{Al}_{2} \mathrm{O}_{3}$ interface defects. Though these devices naturally show initial frequency dispersion, it was not affected with neutron exposure, mainly because of the low neutron temperature of the thermal neutron $(0.0253 \mathrm{eV})$.

\subsubsection{Charge Calculations and Detector efficiency:}

Monte Carlo N-Particle Extended (MCNPX) radiation transport code simulations[32] were performed for similar architectures to estimate the deposited energy and predict the number of e-h pair creation. The deposited energy is derived from the energetic reaction products from the ${ }^{10} \mathrm{~B}$ neutron capture event for the Decaborane/ 
Polystyrene layer. As the ${ }^{7} \mathrm{Li}(1.015 \mathrm{MeV})$ and ${ }^{4} \mathrm{He}(1.777 \mathrm{MeV})$ particle traverse through different regions of the device, they deposit energy which is estimated through MCNPX with respect to the neutron fluence $\left(8.5 \times 10^{8} \mathrm{n} / \mathrm{cm}^{2} / \mathrm{s}\right)$, neutron temperature and the ${ }^{10} \mathrm{~B}$ atom density in the material. This energy is then converted to estimate the e-h pair density at each layer. Table 4-6 shows the simulation results for 0.120um Polystyrene/Decaborane layer. The neutron capture efficiency is highly dependent on the thickness of the converter layer and for a 0.120 um PS-DB layer, it was found to be $0.15 \%$. Typically, the ${ }^{7} \mathrm{Li}$ and ${ }^{4} \mathrm{He}$ charged particles have a traversing range of $2.7-3.0 \mu \mathrm{m}$ and $5.1-6.4 \mu \mathrm{m}$ in decaborane, respectively, and thus for a 0.120 um Ps-DB layer the particles just pass through all the layers depositing energy, but most of it is deposited at the $\mathrm{Si} / \mathrm{Al}_{2} \mathrm{O}_{3}$ interface. In comparison to the predicted charges from MCNPX, the experimental trapped charges from Figure 4-10 are close to predicted charges at the PS-DB layer. (b) shows comparison of detected charges vs. MCNPX simulated charges, where a clear linear relationship is depicted for the number of charges and the neutron dose. The increase in experimental charges with respect to the neutron dose also follows a similar trend predicted through MCNPX. From this plot, the limit of detection for $90 \%$ confidence level was calculated to be $3.95 \mathrm{E}+09 \mathrm{n} / \mathrm{cm}^{2}$.

Figure 4-10 also shows the plot of estimated e-h pair density at each layer and the experimental trapped charges. The close relationship between the number of experimentally detected charges and the charge generation at the PS-DB layer predicts that the charge separation happens at the PS-DB layer (shown in Figure 4-10(b)), where the electrons are tunneling to the $1 \mathrm{~nm}$ Pt NP layer to get trapped and retained. The other two possibilities of charge tunneling to NP can be from the $\mathrm{Si} / \mathrm{Al}_{2} \mathrm{O}_{3}$ interface and the bulk 
$\mathrm{Al}_{2} \mathrm{O}_{3}$. Though charges created at bulk $\mathrm{Al}_{2} \mathrm{O}_{3}$ can get trapped, they are low in number and are negligible compared to the number of charges at $\mathrm{Si} / \mathrm{Al}_{2} \mathrm{O}_{3}$ interface and at PS-DB layer. On the other hand, the charges at the $\mathrm{Si} / \mathrm{Al}_{2} \mathrm{O}_{3}$ are blocked by the $3 \mathrm{~nm} \mathrm{Al}_{2} \mathrm{O}_{3}$ at the interface and ultrafine $0.5 \mathrm{~nm}$ Pt NP with very high Coulomb blockade which does not allow the charge to tunnel. Ultimately, the charges at the PS-DB layer tunnels to $1 \mathrm{~nm} \mathrm{Pt}$ NP shifting the Voltage flat band of the device. This mechanism is graphically illustrated in Figure 4-10 (a)

Table 4-6: Number of e-h pair estimation from MCNPX simulations for different neutron flux

\begin{tabular}{lllll}
\hline $\begin{array}{l}\text { Neutron } \\
\text { Flux } \\
\left(\mathbf{n} / \mathbf{c m}^{\wedge} \mathbf{2}\right)\end{array}$ & $\begin{array}{l}\text { eh pairs } \\
\text { in Si/cm2 } 2\end{array}$ & $\begin{array}{l}\text { eh pairs } \\
\text { in } \\
\text { alumina } \\
\text { in cm2 }\end{array}$ & $\begin{array}{l}\text { eh pairs } \\
\text { from PS- } \\
\text { DB layer }\end{array}$ & $\begin{array}{l}\text { Total } \\
\text { Charges }\end{array}$ \\
\hline $\mathbf{0}$ & 0 & 0 & 0 & 0 \\
$\mathbf{1 . 1 9 E}+\mathbf{1 0}$ & $5.52 \mathrm{E}+12$ & $1.14 \mathrm{E}+10$ & $2.11 \mathrm{E}+11$ & $3.41 \mathrm{E}+13$ \\
$\mathbf{1 . 6 2 E}+\mathbf{1 0}$ & $7.49 \mathrm{E}+12$ & $1.54 \mathrm{E}+10$ & $2.86 \mathrm{E}+11$ & $4.63 \mathrm{E}+13$ \\
$\mathbf{3 . 0 2 E}+\mathbf{1 0}$ & $1.40 \mathrm{E}+13$ & $2.88 \mathrm{E}+10$ & $5.35 \mathrm{E}+11$ & $8.66 \mathrm{E}+13$ \\
$\mathbf{5 . 1 0 E}+\mathbf{1 0}$ & $2.36 \mathrm{E}+13$ & $4.87 \mathrm{E}+10$ & $9.04 \mathrm{E}+11$ & $1.46 \mathrm{E}+14$ \\
\hline
\end{tabular}



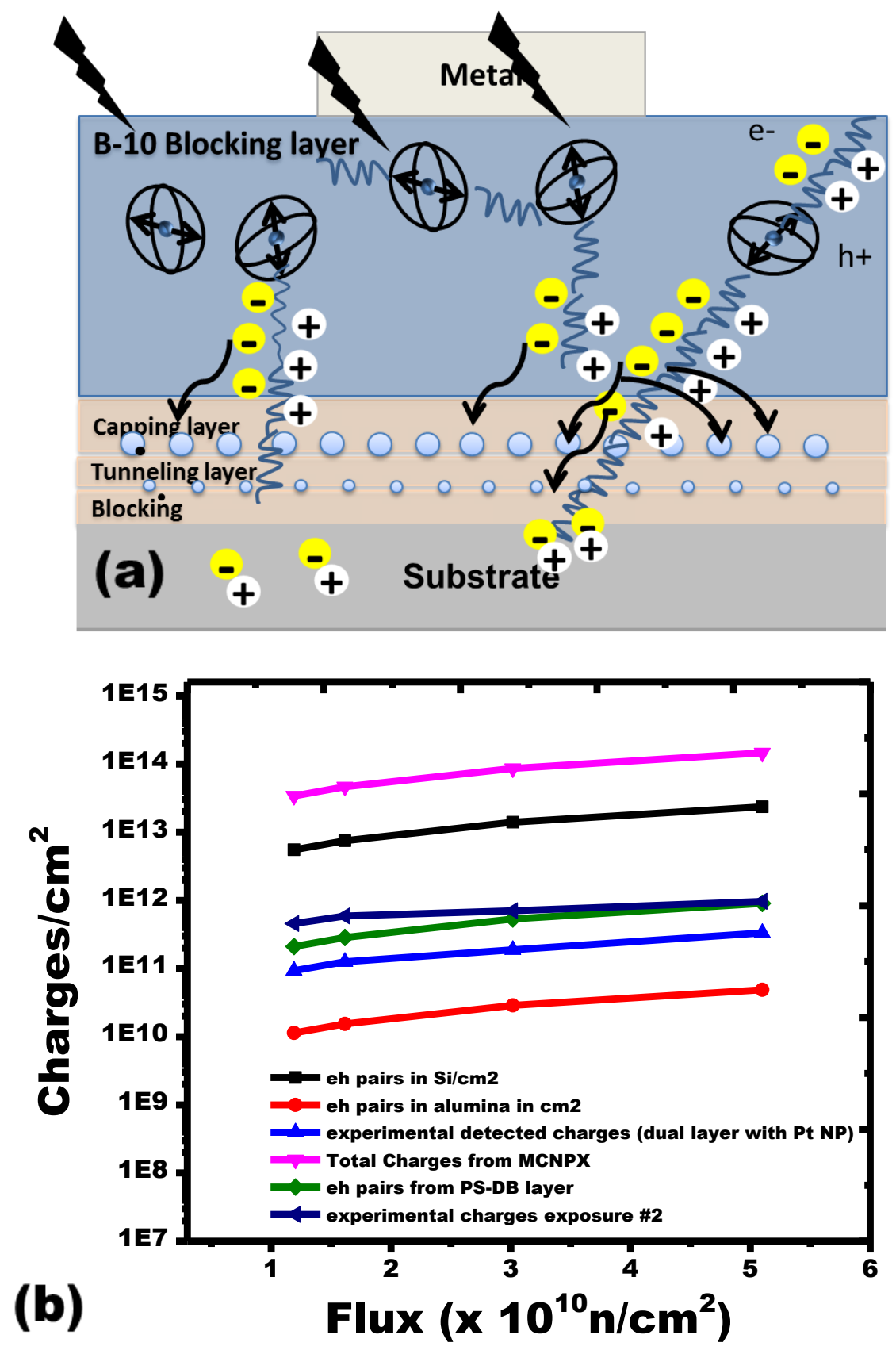

Figure 4-10 (a) Representation of energetic particle interaction with NP embedded device stack; (b)Charge plot vs Neutron dose for experimental (blue, green) vs MCNPX estimations 


\subsubsection{Ionization damage and displacement effects}

To determine the neutron ionization effects occurring at different device layer interfaces and establish that the neutron detection is purely from the combination of ${ }^{10} \mathrm{~B}$ layer and Pt NP and not through ionization damage/effects, devices without any Decaborane layers (Devices schematic shown in Fig 4-2(b)) were exposed to the same neutron dose and studied for bias dependence and frequency dispersion to see any observable effects. Figure 4-11 shows the voltage sweep C-V curves for Si based control and dual layer Pt NP embedded devices for different neutron doses(Fig 4-4(b)). From the $\mathrm{C}-\mathrm{V}$ analysis, there was no change in observable parameters and the devices were unaffected by the thermal neutron dose and the $64 \mathrm{cG} / \mathrm{h}$ Gamma radiation. Frequency dispersion $\mathrm{C}-\mathrm{V}$ curves were also measured to see no observable change for neutron exposure stating no displacement damage at the $\mathrm{Si} / \mathrm{Al}_{2} \mathrm{O}_{3}$ interface. This holds true, since typically neutrons with higher temperature exhibit ionization radiation. Thermal neutron of just $0.023 \mathrm{eV}$ energy is harmless as it is just in the same order of thermal energy at room temperature. For ionization and displacement damage, it takes the neutron to be in the epithermal and fast neutron range. Moreover, since these devices were also exposed to a gamma dose, we believe that these can be potentially used for gamma discrimination. 

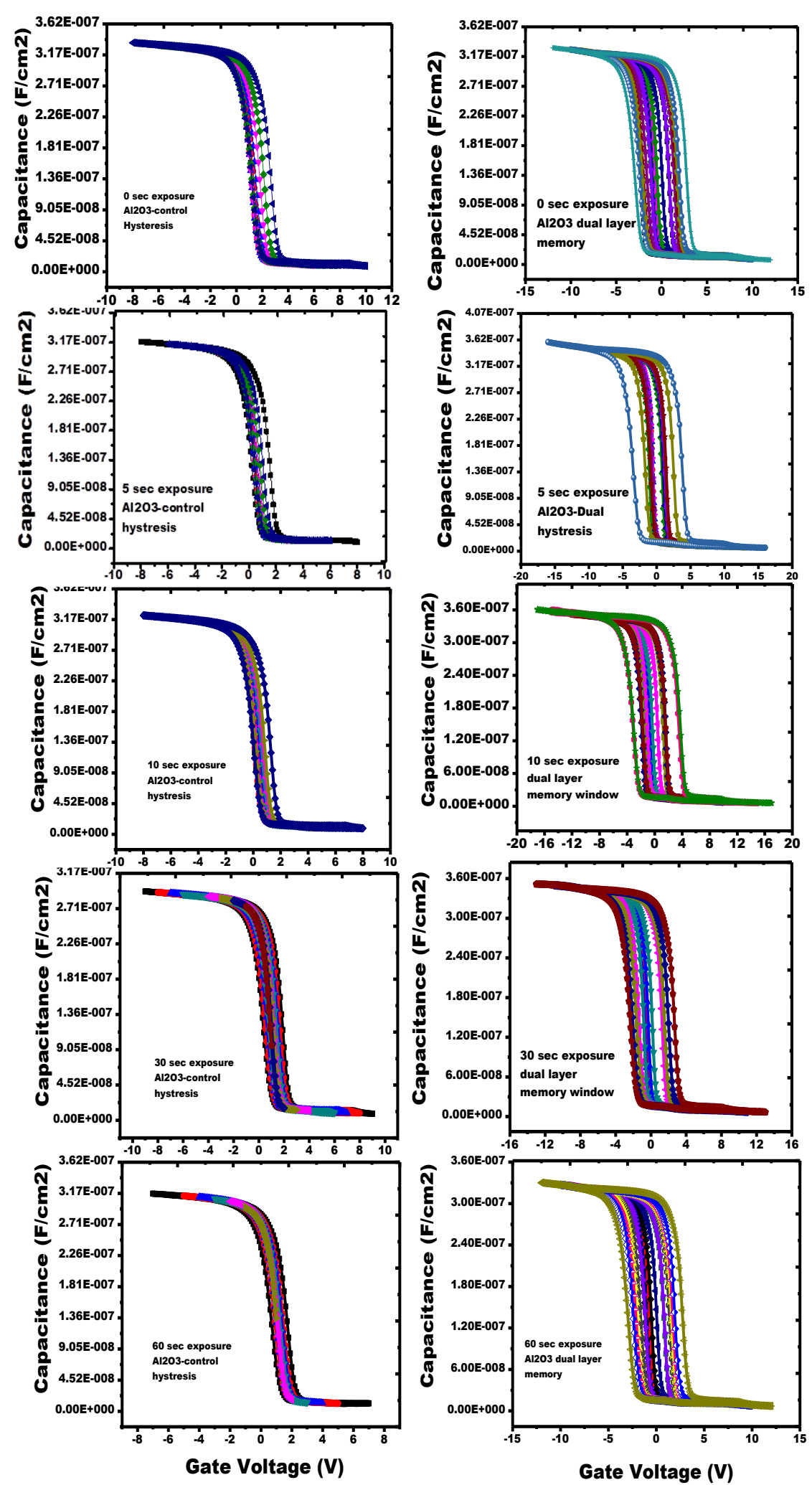

Figure 4-11 voltage sweep C-V curves for Si based control and dual layer Pt NP embedded devices for different neutron doses 


\subsection{REFERENCES}

1. Lakshmanan, A., A review on the rǒle of thermoluminescent dosimeters in fastneutron personnel dosimetry. Nuclear Tracks and Radiation Measurements (1982), 1982. 6(2): p. 59-78.

2. Noulty, R. and T. McLean, Bubble detectors-a maturing technology. Radiation measurements, 1997. 27(1): p. 1-11.

3. Tommasino, L., et al., Different etching processes of damage track detectors for personnel neutron dosimetry. Nuclear Tracks and Radiation Measurements (1982), 1984. 8(1): p. 335-339.

4. Knoll, G.F., Radiation detection and measurement2010: Wiley. com.

5. Flakus, F., Detecting and Measuring lonizing Radiation- A Short History. IAEA Bulletin, 1982. 23(4): p. 31-36.

6. Wehe, D.K., Current Trends in Ionizing Radiation Detection. Nuclear Engineering and Technology, 2006. 38(4): p. 311.

7. Guardiola, C., et al., First investigations of a silicon neutron detector with a carborane converter. Journal of Instrumentation, 2011. 6(11): p. P11001.

8. Caruso, A.N., The physics of solid-state neutron detector materials and geometries. Journal of Physics: Condensed Matter, 2010. 22(44): p. 443201.

9. Caruso, A., et al., The all boron carbide diode neutron detector: Comparison with theory. Materials Science and Engineering: B, 2006. 135(2): p. 129-133.

10. McGregor, D.S. and J. Kenneth Shultis, Reporting detection efficiency for semiconductor neutron detectors: A need for a standard. Nuclear Instruments and 
Methods in Physics Research Section A: Accelerators, Spectrometers, Detectors and Associated Equipment, 2011. 632(1): p. 167-174.

11. McGregor, D., T. Unruh, and W. McNeil, Thermal neutron detection with pyrolytic boron nitride. Nuclear Instruments and Methods in Physics Research Section A: Accelerators, Spectrometers, Detectors and Associated Equipment, 2008. 591(3): p. 530-533.

12. McGregor, D.S., et al., Design considerations for thin film coated semiconductor thermal neutron detectors - I: basics regarding alpha particle emitting neutron reactive films. Nuclear Instruments and Methods in Physics Research Section A: Accelerators, Spectrometers, Detectors and Associated Equipment, 2003. 500(1): p. 272-308.

13. Baumann, R.C. and E.B. Smith. Neutron-induced boron fission as a major source of soft errors in deep submicron SRAM devices. in Reliability Physics Symposium, 2000. Proceedings. 38th Annual 2000 IEEE International. 2000. IEEE.

14. Makowski, D., et al., The application of a SRAM chip as a novel neutron detector. Journal of Experimental Nanoscience, 2006. 1(2): p. 261-268.

15. Baumann, R., Soft errors in commercial integrated circuits. International Journal of High Speed Electronics and Systems, 2004. 14(02): p. 299-309.

16. Sax, K., et al., Evaluation of an SRAM-Based Thermal Neutron-Detection System.

17. Ramalingam, B., et al., Sub-2 $\mathrm{nm}$ size and density tunable platinum nanoparticles using room temperature tilted-target sputtering. Nanotechnology, 2013. 24(20): p. 205602. 
18. Yun, M., B. Ramalingam, and S. Gangopadhyay, Multi-Layer Pt Nanoparticle Embedded High Density Non-Volatile Memory Devices. Journal of The Electrochemical Society, 2012. 159(4): p. H393-H399.

19. Yun, M., B. Ramalingam, and S. Gangopadhyay, Room temperature observation of size dependent single electron tunneling in a sub-2 $\mathrm{nm}$ size tunable Pt nanoparticle embedded metal-oxide-semiconductor structure. Nanotechnology, 2011. 22(46): p. 465201.

20. Minseong, Y., et al., Sub-2 nm Size-Tunable High-Density Pt Nanoparticle Embedded Nonvolatile Memory. Electron Device Letters, IEEE, 2009. 30(12): p. 1362-1364.

21. Jeff, J.R.C., et al., Charge storage characteristics of ultra-small Pt nanoparticle embedded GaAs based non-volatile memory. Applied Physics Letters, 2011. 99(7): p. 072104-3.

22. Zheng, H., et al., Large sensitivity enhancement in semiconducting organic field effect transistor sensors through incorporation of ultra-fine platinum nanoparticles. Applied Physics Letters, 2013. 103(19): p. --

23. An, W.-J., et al., Role of Pt Nanoparticles in Photoreactions on TiO2 Photoelectrodes. MRS spring meeting and Exhibit, 2012.

24. Ishizaka, A. and Y. Shiraki, Low temperature surface cleaning of silicon and its application to silicon MBE. Journal of The Electrochemical Society, 1986. 133(4): p. 666-671. 
25. Puurunen, R.L., Surface chemistry of atomic layer deposition: A case study for the trimethylaluminum/water process. Journal of Applied Physics, 2005. 97(12): p. 121301.

26. Sze, S.M. and K.K. Ng, Physics of semiconductor devices2006: John Wiley \& Sons.

27. Cotton, F.A., et al., Advanced inorganic chemistry. Vol. 5. 1988: Wiley New York.

28. ; Available from: http://www.csgnetwork.com/dieconstantstable.html.

29. Minseong, Y., R. Balavinayagam, and G. Shubhra, Room temperature observation of size dependent single electron tunneling in a sub-2 $\mathrm{nm}$ size tunable Pt nanoparticle embedded metal-oxide-semiconductor structure. Nanotechnology, 2011. 22(46): p. 465201.

30. Ramalingam, B., H.Zheng, and S. Gangopadhyay, Multi-Bit Operation in NonVolatile Memory Devices using Controlled Charging Behavior for Double-Layer Floating Gate Devices. under preparation, 2013.

31. Durrani, Z.A.K., Single-electron devices and circuits in silicon2010: World Scientific.

32. Labrotaries, L.A.N., MCNPX, 2013, LANL. 


\section{CHAPTER 5: Conclusions and Future Work}

\subsection{Conclusion}

To conclude, sputtered sub-2 nm Pt nanoparticle growth characteristics by tilted-target deposition were studied in this work by varying the target angle, power and time. By simply optimizing the parameters, the size and density of the particles can be controlled so precisely that the mean diameter can be shifted in intervals of less than $0.2 \mathrm{~nm}$ and the density can be varied from $1 \mathrm{E} 12$ to $1 \mathrm{E} 13 / \mathrm{cm}^{2}$. Three separate nanoparticle growth regimes (nucleation dependent, coalescence dependent and agglomeration) were observed based on the detailed analysis of HRTEM images of these nanoparticles.

It was shown that, with time, nucleation dependent growth dominates at the beginning stage of nanoparticle growth and is replaced by diffusion and coalescence based growth, which is further followed by agglomeration at later growth stages. The sputtering power is another important parameter, and when combined with time can be used to precisely control the size, density and size distribution of the particles. The size dependent crystalline characteristics of these nanoparticles were also studied, and it was concluded that at very low sputtering time domains $(\mathrm{t}<20 \mathrm{~s})$ the particles are non-crystalline clusters, whereas they evolve over time to form single crystalline nanoparticles and finally polycrystalline $\mathrm{Pt}$ nano-islands further down the sputtering time domain.

Detailed understanding of various nanoparticle growth domains provides one a unique opportunity to design unique nanoparticle decorated surfaces (with desired nanoparticle size and number density) for a variety of applications. For example, in the case of charge 
retention applications, small homogeneous non-crystalline nanoparticles with large interparticle distances are preferred. These features can be attained by carrying out depositions within the nucleation regime and using high energy particles (lower target angles/lower deposition power) to attain smaller size distributions and high inter-particle distances (lower nanoparticle number densities). Meanwhile, for catalysis applications, crystalline nanoparticles with low inter-particle distances (high jamming limit) would be ideal. In this case, high target angle depositions could be carried out first in order to attain high number densities, followed by subsequent low target angle deposition or substrate annealing to attain larger crystalline particles with relatively narrow size distribution. This matrix of information was instrumental in applications where the size and density of Pt nanoparticles needed to be tuned in the sub- $2 \mathrm{~nm}$ ranges.

For example, in Chapter 3 we saw that how the size and density were important factors in order to observe layer by layer charging. Controlled layer-by-layer charging was demonstrated using the optimized double-layer floating gate NVM MOSCAPs with embedded size- and density-tunable PtNPs. Test structures with appropriate PtNP configuration and specific tunneling and interparticle dielectric layer thicknesses revealed a distinct step effect in the programming memory window. Simply using two high-density PtNP layers resulted in a more pronounced step effect and a large $15 \mathrm{~V}$ memory window compared to Si quantum dot-based structures. The importance of precise control over each parameter in the device architecture to achieving the step effect phenomenon was driven home by providing and explaining contrary device results using non-optimal particle and dielectric configurations in terms of the mechanisms of device failure and potential methods to overcome these shortfalls through further modification of the device 
configuration. These observations suggest the feasibility of using multi-level architecture in the same device architecture. The high retention properties and high charging energies observed in these devices gave us a good background to make devices for neutron detection sensors. Novel dual layer Pt NP embedded device architectures with $0.52 \pm 0.12 \mathrm{~nm}$ and $1.11 \pm 0.28 \mathrm{~nm}$ PtNPs and PS/DB capping layers were fabricated and exposed to thermal neutrons (greater than $1 \mathrm{e} 10 \mathrm{n} / \mathrm{cm}^{2}$ ) and gamma to determine a characteristic neutron sensing mechanism. These devices are based on a direct-dielectric configuration trapping e-h pairs generated in the active layer of the device (through energetic particle from neutron capture at ${ }^{10} \mathrm{~B}$ layer) and producing a signal relevant to a detection event. The charge/ $\mathrm{cm}^{2}$ trapped in these devices are proportional to the neutron fluence making it an efficient detection scheme. Other control experiments and samples were also tested to deduct the working principle of these detectors, where control sample and single layer devices with $1.11 \mathrm{~nm}$ and $1.45 \mathrm{~nm}$ Pt NP did not exhibit neutron detection . Overall, a unique and sensitive neutron detection scheme based on direct-conversion architecture, with capability of gamma discrimination was exhibited. We believe that, with MOSFET architectures these devices can exhibit promising neutron sensing mechanism that has great potential

\subsection{Future Direction}

The future work for this doctoral dissertation will be in fabricating NVM MOSFET device architectures with boron enriched dielectrics. Some of the proposed directions are:

\subsubsection{Incorporating metal NP in ${ }^{10} \mathrm{~B}$ polymer:}

We propose a novel method to incorporate gold and platinum metal nanoparticles (NP) as charge trap centers embedded in ${ }^{10} \mathrm{~B}$-enriched polymer insulator layer for Metal Oxide 
Semiconductor Capacitor (MOSCAP) and Metal oxide semiconductor field effect transistor (MOSFET) architectures. The NP-embedded ${ }^{10} \mathrm{~B}$ enriched polymer layer act to both neutron capture as well as to hold generated charges for signal transduction. Thermal neutrons absorbed by ${ }^{10} \mathrm{~B}$ produce ${ }^{7} \mathrm{Li}(1.015 \mathrm{MeV})+{ }^{4} \mathrm{He}(1.777 \mathrm{MeV})$ with a branching ratio of $94 \%$ and ${ }^{7} \mathrm{Li}(0.84 \mathrm{MeV})+{ }^{4} \mathrm{He}(1.470 \mathrm{MeV})+0.482 \mathrm{MeV}$ gamma ray with a branching ratio of $6 \%$. Based on our MCNPX analysis, in our ${ }^{10} \mathrm{~B}$-enriched polymer insulator layer, the ${ }^{7} \mathrm{Li}$ and ${ }^{4} \mathrm{He}$ are charged particles with a range of $2.7-3.0 \mu \mathrm{m}$ and 5.1 $-6.4 \mu \mathrm{m}$, respectively. A detection event will occur when a decelerating charged particle generated upon neutron capture (through ${ }^{10} \mathrm{~B}$ ) creates electron-hole pairs in or at the interface to the converter region of the device. Most importantly, the embedded ultra-fine Pt nanoparticles play dual role of separating the e-h pairs generated by primary reaction products of a neutron capture event and trapping electrons due to strong Coulomb blockade effect. The number of trapped charges can then be quantified by the change in device characteristics such as flat band voltage shift which would correspond to a neutron dose incident on the detector. $\mathrm{A}{ }^{10} \mathrm{~B}$ based insulator material can be synthesized using the crosslinking of novel boron containing monomers with styrene or methacrylate forming highly-cross-linked polymers containing up to $45 \%{ }^{10} \mathrm{~B}$ by weight. Polystyrene and polymethacrylate have been shown to possess high thermal, oxidative, and hydrolytic stability, as well as, superior radiation hardness properties. The metal nanoparticle embedded ${ }^{10} \mathrm{~B}$ polymer layer will act as the gate dielectric for the fabrication of MOSCAP and MOSFET devices with two choices of semiconductors: silicon for ease of fabrication for CMOS compatibility; and indium Gallium Zinc Oxide (InGaZnO) due to it's large band gap and radiation hardness. Finally, $\mathrm{Si}$ and InGaZnO based MOSCAP and MOSFET will 
be studied along with the NP embedded ${ }^{10} \mathrm{~B}$ polymer for ionization effects and irreversible damage at interfacial sites for neutron and gamma radiation.

\subsubsection{Approach:}

To increase the charge trapping efficiency in the proposed novel materials, we will incorporate characteristically sized and distributed metal NP in the ${ }^{10} \mathrm{~B}$ enriched insulator. This will aid in tuning the Coulomb charging efficiency of the NP resulting in optimum charge trapping and retention. We propose two methods to achieve this:

\subsection{2a: Physical methods (Shubhra Gangopadhyay): Evaporating/sputtering}

metal on ${ }^{10} \mathrm{~B}$ thin-film kept at $\mathrm{T}_{\mathrm{g}}$ and then cooled down for metal atom diffusion and coagulation. This study will be performed at different ${ }^{10} \mathrm{~B}$ compositions kept at different temperatures to study the metal atom diffusion, nucleation and growth. Experimental analysis with detection results on these insulating devices will further help understanding charge separation using directconversion hetero-structures and the

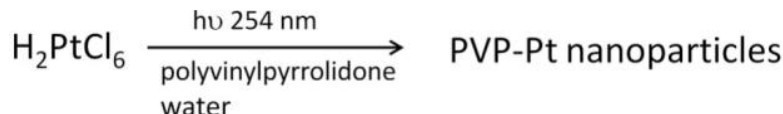
Scheme 3. The solution phase synthesis of $1 \mathrm{~nm}$ platinum nanoparticles importance of NP size and density $\begin{gathered}\text { Scheme } 3 . \\ \text { in water. }\end{gathered}$ towards detection.

5.2.2b: Solution phase synthesis of platinum nanoparticles: An alternative method for the synthesis of sub-2nm nm diameter platinum nanoparticles can be performed in a manner similar to that previously reported. Briefly, as aqueous solution of chloroplatinic acid can be added to polyvinylpyrrolidone and irradiated for one hour using $254 \mathrm{~nm}$ ultraviolet light (Scheme 3) to for Pt NP. These Pt nanoparticles coated with 
polyvinylpyrrolidone will be dispersed in carbomethyl acrylate monomer with different concentrations and polymerized.

\subsubsection{Silicon on Insulator Devices and sputtered InGaZnO}

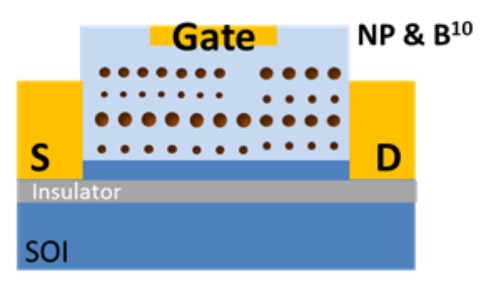

Figure 5-1: SOI- based Boron and NP enriched MOSFET

Silicon on insulator devices and sputtered InGaZnO based MOSFET can be used to incorporate the synthesized novel materials to study other device parameters as well as understand the in-situ reactions during neutron exposure events. MOSFET structures (Figure 5-1) are especially interesting for such applications because of the number of parametric extraction possible. By real time monitoring of multiple OFET parameters, such as the drain current, change of mobility and hysteresis window, high sensitivity can be observed instrumental in determining factors like Limit of detection, etc. Especially with our unique Pt NP and its specific capacitance for a high coulomb blockade effect, a neutron detection event can be effectively transduced into collecting and separating charges. These NP tend to trap e-s whenever possible in multiple-diverse scenarios, for eg, our recent study on their characteristic behavior has shown single electron tunneling behavior in Si solidstate devices, as well as e- trapping for rapid transfer of e-s to absorbed $\mathrm{CO} 2$ vapor during a photoreduction process [5]. Due to the thin geometry of these devices, we expect the 
Gamma radiation to just pass through without inducing any effect. This gamma discrimination can also be studied and explored. 


\section{List of Publications}

[1] B. Ramalingam, H.Zheng, and S. Gangopadhyay, "Multi-Bit Operation in NonVolatile Memory Devices using Controlled Charging Behavior for Double-Layer Floating Gate Devices," under review, Applied Physics letters, 2014.

[2] B. Ramalingam, S. Mukherjee, C. J. Mathai, K. Gangopadhyay, and S. Gangopadhyay, "Sub-2 nm size and density tunable platinum nanoparticles using room temperature tilted-target sputtering," Nanotechnology, vol. 24, p. 205602, 2013.

[3] H. Zheng, R. Balavinayagam, V. Korampally, and G. Shubhra, "Large sensitivity enhancement in semiconducting organic field effect transistor sensors through incorporation of ultra-fine Platinum nanoclusters," Applied Physics Letters, vol. submitted, 2013.

[4] S. Mukherjee, B. Ramalingam, L. Griggs, S. Hamm, G. A. Baker, P. Fraundorf, S. Sengupta, and S. Gangopadhyay, "Ultrafine sputter-deposited Pt nanoparticles for triiodide reduction in dye-sensitized solar cells: impact of nanoparticle size, crystallinity and surface coverage on catalytic activity," Nanotechnology, p. In Print, 2012.

[5] M. Yun, B. Ramalingam, and S. Gangopadhyay, "Multi-Layer Pt Nanoparticle Embedded High Density Non-Volatile Memory Devices," Journal of The Electrochemical Society, vol. 159, pp. H393-H399, 2012.

[6] M. Yun, B. Ramalingam, and S. Gangopadhyay, "Room temperature observation of size dependent single electron tunneling in a sub-2 $\mathrm{nm}$ size tunable Pt nanoparticle 
embedded metal-oxide-semiconductor structure," Nanotechnology, vol. 22, p. 465201, 2011.

[7] J. R. C. Jeff, M. Yun, B. Ramalingam, B. Lee, V. Misra, G. Triplett, and S. Gangopadhyay, "Charge storage characteristics of ultra-small Pt nanoparticle embedded GaAs based non-volatile memory," Applied Physics Letters, vol. 99, pp. 072104-3, 2011.

[8] W.-J. An, W.-N. Wang, B. Ramalingam, S. Mukherjee, B. Daubayev, S. Gangopadhyay, and P. Biswas, "Role of Pt Nanoparticles in Photoreactions on TiO2 Photoelectrodes," MRS spring meeting and Exhibit, 2012.

[9] W.-N. Wang, W.-J. An, B. Ramalingam, S. Mukherjee, D. M. Niedzwiedzki, S. Gangopadhyay, and P. Biswas, "Size and Structure Matter: Enhanced CO2 Photoreduction Efficiency by Size-resolved Ultrafine Pt Nanoparticles on TiO2 Single Crystals," Journal of the American Chemical Society, 2012. 


\section{VITA}

Balavinayagam Ramalingam was born in New Delhi, India on 27th February 1986. He received his Bachelor in Engineering in Electronics and Communication from Anna University, Chennai, India in 2007 where he researched about Phase shifted QPSK modulators. In May 2009, he earned his Masters degree in Department of Electrical and Computer Engineering at University of Missouri, under the advicement of Dr.Justin Legarsky. Shortly after his masters, he enrolled into the Doctoral program and researched upon nanoparticle growth and nonvolatile memory devices under the guidance of Dr. Shubhra Gangopadhyay. He will be graduating with his Ph.D degree in Electrical Engineering in Spring 2014 from the same department. He will join as a Process Tecnology Development Engineer in Intel Corporation, at Portland, Oregon. 